\title{
Transformationsprozesse der landwirtschaftlichen Nutztierhaltung \\ Gesellschaftliche Ansprüche und Gestaltungsmöglichkeiten des Lebensmitteleinzelhandels
}

\author{
Dissertation \\ zur Erlangung des Doktorgrades (Dr. sc. agr.) \\ der Fakultät für Agrarwissenschaften \\ der Georg-August-Universität Göttingen
}

vorgelegt von

Maureen Schulze

geboren in Hamburg

Göttingen, im Juli 2020 
D 7

1. Referent: $\quad$ Prof. Dr. Achim Spiller

2. Referent: Prof. Dr. Ulrich Enneking

3. Referent Prof. Dr. Guido Recke

Tag der mündlichen Prüfung: 22. September 2020 


\section{Zusammenfassung}

Die moderne landwirtschaftliche Nutztierhaltung gerät zunehmend in die Kritik, denn technischer Fortschritt und Effizienzsteigerungen gehen mit Tier-, Umwelt- und Naturschutzproblemen einher. Gesellschaftlich wird daher zunehmend eine Transformation der bestehenden Produktions- und Haltungsbedingungen gefordert. Die vorliegende Dissertation betrachtet dabei die gesellschaftlichen Anforderungen an einen Transformationsprozess sowie die Gestaltungsmöglichkeiten des Lebensmitteleinzelhandels.

Gesellschaftliche Unzufriedenheit mit modernen Produktionspraktiken der landwirtschaftlichen Nutztierhaltung äußert sich zunehmend in sozialen Protestbewegungen, wie z.B. Demonstrationen oder Stallbauprotesten. In jüngster Zeit haben insbesondere heimlich erstellte Aufnahmen von Tierschützern, die nachts in Ställe eindringen, um die Produktionsund Haltungsbedingungen $\mathrm{zu}$ dokumentieren mediale Aufmerksamkeit erlangt. Bisher ist unklar, ob derartige Protestformen als legitim wahrgenommen werden und welche Treiber der gesellschaftliche Einstellungsbildung zugrunde liegen und soll daher im ersten Teil der vorliegenden Dissertation im Kapitel „Gesellschaftliche Wahrnehmung heimlicher Stallaufnahmen“ untersucht werden. Im Rahmen von zwei aufeinander aufbauenden Beiträgen wird die gesellschaftliche Wahrnehmung heimlicher Aufnahmen erfasst. Die Ergebnisse zeigen, dass heimliche Aufnahmen gesellschaftlich auf hohen Zuspruch treffen und eine härtere strafrechtliche Ahndung eher abgelehnt wird. Heimliche Aufnahmen werden als geeignete Maßnahme angesehen, um Tierschutzvergehen aufzudecken. Sie gelten aber auch als glaubwürdige Informationsquelle, um Einblicke in die landwirtschaftliche Nutztierhaltung, die vermehrt hinter verschlossenen Türen stattfindet, zu erlangen.

Die Transformation hin zu höheren Tier-, Umwelt,- oder/und Naturschutzstandards geht unweigerlich mit höheren Produktionskosten einher. Gesellschaftliche Erwartungen müssen daher in individuelle Konsumentscheidungen umgewandelt werden. Dafür ist ein weitreichendes Verständnis über Konsumpräferenzen und Zahlungsbereitschaften wichtig. Das Kapitel „Konsumentenpräferenzen und Zahlungsbereitschaften für nachhaltig erzeugte Fleischwaren“ der vorliegenden Dissertation widmet sich daher im Rahmen eines Beitrags der Ermittlung von Konsumentenpräferenzen und Zahlungsbereitschaften von Weiderindfleisch. Die Untersuchung zeigt sich, dass Weidehaltung einen entscheidenden Einfluss auf die Kaufentscheidung hat. Aber auch die Nutzung von weniger intensiv gezüchteten Rassen beeinflusst die Kaufentscheidung positiv. Daraus lassen sich Produktdifferenzierungs- 
möglichkeiten ableiten, die vor allem tierfreundliche Prozessattribute in den Vordergrund stellen.

Als wichtigster Einkaufsort für Fleischwaren kann der Lebensmitteleinzelhandel die Konsumentscheidungen maßgeblich beeinflussen. Im Rahmen des dritten Kapitels der vorliegenden Dissertation mit dem Titel „Der Lebensmitteleinzelhandel als Gestalter nachhaltiger Konsum- und Produktionsmuster“" werden daher die Motive und Hindernisse des Lebensmitteleinzelhandels, sich für eine Vermarktung nachhaltig erzeugter Fleischwaren einzusetzen, untersucht. Dies wird im Rahmen von zwei aufeinander aufbauenden Beiträgen, ebenfalls am Beispiel von der Vermarktung von Weiderindfleisch, beleuchtet. Es zeigt sich, dass Lebensmitteleinzelhändler Aufgaben übernehmen müssen, die sonst von der Industrie getragen werden. Gleichzeitig müssen aufwendige Marketingmaßnahmen entwickelt werden, um den Kunden den Mehrwert des Produktes vermitteln zu können. Finanzielle Anreize allein können diesen Mehraufwand nicht erklären. Vor allem das Interesse am Wohlergehen landwirtschaftlicher Nutztiere, sowie Umwelt- und Naturschutz aber auch das Interesse an der Unterstützung der ländlichen Entwicklung motiviert Lebensmitteleinzelhändler Weiderindfleisch in ihre Produktsortiment aufzunehmen.

Die vorliegende Dissertation lässt erkennen, dass der gesellschaftliche Druck auf die moderne Nutztierhaltung immer weiter zunimmt. Eine Anpassung der Kommunikationsstrategie ist längst nicht mehr ausreichend, um eine gesellschaftlich akzeptierte Nutztierhaltung zu entwickeln. Vielmehr müssen tatsächliche Anpassungen der Produktionsbedingungen vorgenommen werden. Eine gesellschaftliche Forderung nach möglichst natürlichen Haltungsbedingungen ist aber für die Anzahl der momentan in Deutschland gehaltenen Tiere aus Umwelt- und Naturschutzgründen nicht möglich. Eine mögliche Lösung ist die Reduktion der Tierbestände. Eine Reduktion der Tierbestände würde allerdings mit einer Anpassung der bisher im Fleischsektor angewendeten Strategie der Kostenführerschaft erfordern. Begleitet durch eine Differenzierungsstrategie, die vor allem nachhaltige Produktionsbedingungen in den Vordergrund stellt, kann der Transformationsprozess zu einer gesellschaftlich akzeptierten Nutztierhaltung führen. 


\section{Inhaltsverzeichnis}

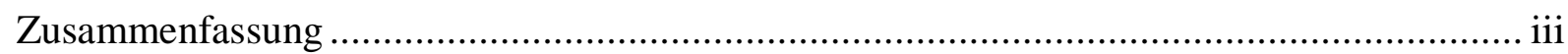

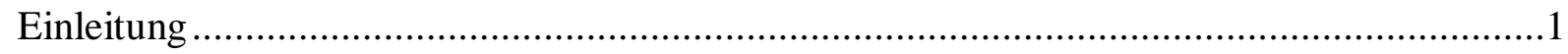

Teil I - Gesellschaftliche Wahrnehmung heimlicher Stallaufnahmen ....................................13

I.1 Heimliche Stallaufnahmen aus gesellschaftlicher Sicht im Wechselspiel zwischen Landwirtschaft, Tierschutzorganisationen und staatlichen Kontrollmechanismen

I.2 Public Perceptions of Undercover Investigations in Livestock Farming: An End that Justifies the Means?

Teil II - Präferenzen und Zahlungsbereitschaften für nachhaltig erzeugte Fleischwaren

II.1 Do consumers prefer pasture-raised dual-purpose cattle when considering meat products? A hypothetical discrete choice experiment for the case of minced beef.

Teil III - Der Lebensmitteleinzelhandel als Gestalter nachhaltiger Konsum- und Produktionsmuster.

III.1 Food Retailers as Mediating Gatekeepers between Farmers and Consumers in the Supply Chain of Animal Welfare Meat - Studying Retailers' Motives in Marketing Pasture-Based Beef

III.2 Challenges and Motives in Food Retail Cooperatives' Approach to

Supporting Alternative Niche Paths Using the Case of Locally Produced

Grass-Fed Beef. .61

Resümee und Ausblick .85

Veröffentlichungs- und Votragsverzeichnis .94

Beiträge der Autoren .96

Eidesstattliche Erklärung .98 


\section{Einleitung}

Geprägt von effizienten Produktionsverfahren, werden in der modernen Landwirtschaft standardisierte Lebensmittel zu möglichst günstigen Preisen produziert (Godfray \& Garnett, 2014). Von besonderer Bedeutung in Deutschland ist dabei die Nutztierhaltung, denn knapp $70 \%$ aller Betriebe sind tierhaltend (Statistisches Bundesamt, 2010) und diese generieren mehr als die Hälfte der landwirtschaftlichen Verkaufserlöse (BMEL, 2019). In den letzten Jahren ist die Nutztierhaltung vermehrt in die Kritik geraten, denn Effizienzsteigerungen und Intensivierung der Produktion gehen vielfach mit Tier- sowie Umwelt- und Naturschutzproblemen einher (Godfray \& Garnett, 2014; Rojas-Downing et al., 2017). Eine Transformation der modernen Nutztierhaltung, die mit drastischen Veränderungen einhergeht scheint daher nötig, denn allein technischer Fortschritt oder kleinere Anpassungen scheinen die vielfältigen Zielkonflikte nicht nachhaltig lösen zu können (Koehler et al., 2019). Die Nachhaltigkeitsforschung beschreibt eine erfolgreiche Transformation dabei als eine systemische Anpassung, die durch das Zusammenspiel verschiedener Akteure initiiert wird (Geels \& Schot, 2007). In der vorliegenden Dissertation werden die gesellschaftlichen Anforderungen an den Transformationsprozess, Konsumentenpräferenzen und die Gestaltungsmöglichkeiten des Lebensmitteleinzelhandels näher betrachtet.

Für eine nachhaltige und zukunftsfähige landwirtschaftliche Nutztierhaltung ist vor allem die gesellschaftliche Akzeptanz von besonderer Bedeutung, denn gesellschaftliche Unzufriedenheit gefährdet die ,license to produce“ des Sektors (Vanonacker \& Verbeke, 2014). Eine wachsende Bevölkerungsgruppe steht den Produktionspraktiken der modernen landwirtschaftlichen Nutztierhaltung kritisch gegenüber. Auswirkungen der Tierhaltung auf das Wohlergehen landwirtschaftlicher Nutztiere stehen dabei im Kern der gesellschaftlichen Diskussionen, aber auch Umwelt-, und Naturschutzbedenken gewinnen zunehmend an gesellschaftlicher Relevanz (Clark et al., 2017; Sanchez-Sabate \& Sabaté, 2019; Vanonacker \& Verbeke, 2014). Im Zuge der Urbanisierung hat sich ein Großteil der Gesellschaft mehr und mehr von der landwirtschaftlichen Nutztierhaltung entfernt (Sandøe et al., 2016; Vizzier Thaxton et al., 2016). Nur noch ein geringer Anteil der Bevölkerung hat direkten Kontakt zur Landwirtschaft (Zander et al., 2013). Der Umgang mit Tieren ist in vielen Teilen der Bevölkerung nur noch durch Haustiere bekannt, zu denen eine familiäre Bindung aufgebaut wird (Sandøe et al., 2016). Die intensivierte Beziehung zwischen Haustier und Mensch wird zunehmend auch auf die Haltung von Nutztieren übertragen (Vanonacker \& Verbeke, 2014). Eine ethisch tragbare Nutzung von Tieren wird daher von vielen Menschen als vertretbar 
angesehen, aber nur, wenn Tieren im Gegenzug ein gutes Leben ermöglicht wird (Hölker et al., 2019). Kern der gesellschaftlichen Forderungen ist dabei eine möglichst natürliche Ausgestaltung der Haltungssysteme (Boogaard et al., 2011; Spooner et al., 2014). Dazu gehört ein ausreichendes Platzangebot, das Ausleben natürlicher Verhaltensweisen und ein möglichst schmerzfreies Leben (Boogard et al., 2011; Borkfelt et al., 2015; Lagerkvist et al., 2006). Diese Forderungen widersprechen zum Teil der Realität auf landwirtschaftlichen Betrieben, denn der landwirtschaftliche Strukturwandel und technische Fortschritt haben dazu geführt, dass ein Großteil der landwirtschaftlichen Nutztiere heutzutage in Stallhaltungssystemen gehalten werden (Statistisches Bundesamt, 2010), die teilweise haltungsbedingte Defizite aufweisen und das Ausleben natürlicher Verhaltensweisen einschränken (Rouhda-Mülleder et al., 2010; Schneider et al., 2020). Einseitige Zuchtziele gehen zudem mit gesundheitlichen Problemen der Nutztiere, (z.B. Zusammenhang zwischen hoher Milchleistung und dem Auftreten von Mastitis) (Koeck et al., 2014) sowie einem geringen ökonomischen Wert der männlichen Tiere einher (z.B. Umgang mit männlichen Kälbern aus Milchviehhaltung) (Bodderas \& Graw, 2019). Nicht-kurative Eingriffe am Tier stellen einen Eingriff in die Integrität des Tieres dar (z.B. Enthornen von Kälbern) (Robbins et al., 2015).

Die Differenz zwischen gesellschaftlichen Erwartungen und tatsächlichen Produktionsbedingen sorgt für ein Spannungsverhältnis und schlägt sich zunehmend auch in sozialen Protestbewegungen, wie z.B. Demonstrationen der, wir haben es satt` - Bewegung (www.wir-haben-es-satt.de), Proteste gegen Stallbauvorhaben (Schlecht et al., 2010) oder emotional geladenen und zumeist negativ besetzen Medienberichten (Kayser et al., 2011) nieder. Jüngst haben verstärkt auch teilweise illegale Protestformen, wie heimlich gemachte Aufnahmen aus landwirtschaftlichen Ställen mediale und gesellschaftliche Aufmerksamkeit erlangt (Associated Press, 2017; FAKT, 2019; Lenfers, 2019). Tierschützer ${ }^{1}$ dringen dazu ohne Wissen des Landwirtes in Ställe ein und dokumentieren die Haltungs- und Produktionsbedingungen durch Bild- oder Videoaufnahmen. Im Anschluss wird das Material über die Medien verbreitet und stellt damit eine gesellschaftliche Informationsquelle über die moderne Landwirtschaft dar, die sonst vor allem hinter verschlossenen Türen stattfindet. Die Legitimität derartiger Protestformen ist strittig. Nichtregierungsorganisationen sehen in ihnen eine Möglichkeit Tierschutzvergehen aufzudecken (Johnson, 2014). Landwirte hingegen sehen in ihnen einen Eingriff in die Privatsphäre. Sie sprechen sich einheitlich gegen heimliche Aufnahmen aus und fordern eine härtere strafrechtliche Ahndung (BBV, 2016; Krüsken, 2017; Tonsor \& Olynk, 2010). Auf politischer Ebene ist ebenfalls eine härtere strafrechtliche

\footnotetext{
${ }^{1}$ Hier als Synonym für TierschützerInnen und TierrechtlerInnen verwendet.
} 
Ahndung geplant, wenn auch bisher nicht umgesetzt (Koalitionsvertrag, 2018). Wie heimlich gemachte Aufnahmen in der Gesellschaft wahrgenommen werden ist bisher unbekannt. Wissen über die Treiber der gesellschaftlichen Legitimität und der strafrechtlichen Ahndung der teilweise gesetzeswidrigen Protestformen können aber einen wichtigen Beitrag zu einem besseren Verständnis der gesellschaftlichen Anforderungen an den Transformationsprozess leisten.

Gesellschaftlicher Druck und soziale Protestbewegungen können systemische Anpassungen initiieren (Geels \& Schot, 2007). Neben der Gesellschaft ist auch die individuelle Konsumentscheidung von Bedeutung, denn werden die höheren Produktionskosten im Zuge einer Transformation der modernen Nutztierhaltung nicht indirekt durch Steuern oder Subventionen getragen, können Mehrkosten durch höhere Produktpreise direkt durch den Konsumenten getragen werden. Bisherige wissenschaftliche Untersuchungen deuten auf ein enormes Marktpotenzial von Fleischwaren aus Betrieben mit höheren Tierwohlstandards hin (Clark et al., 2017). Auswirkungen auf Umwelt- und Naturschutz spielen aus Verbrauchersicht beim Fleischkonsum bisher eine untergeordnete Rolle (Sanchez-Sabate \& Sabaté, 2019). Um Marktpotenziale abschöpfen zu können sind Erkenntnisse über Konsumentenpräferenzen und Zahlungsbereitschaften von Relevanz, denn bisher ist der Marktanteil von nachhaltig erzeugten Fleischwaren in Deutschland gering (Moewius et al., 2018; Pirsich et al., 2017).

Dem LEH, als Akteur zwischen Landwirtschaft und Verbraucher, kommt eine entscheidende Rolle zu, wenn es darum geht Konsum- und Produktionsmuster nachhaltig zu beeinflussen. Seine traditionelle Rolle als passiver „Verteiler“ von Lebensmitteln, in der er Fleischwaren lediglich vom Produzenten an seine Kunden weiterreicht, hat der LEH weitestgehend verlassen (Gilbert, 2003). Er agiert nun vermehrt aktiv und versucht mit eigenen Marketingmaßnahmen Konsumentscheidungen zu beeinflussen (Peattie \& Peattie, 2009; Sands et al., 2009). Im Zuge der wachsenden Marktkonzentration zeichnet sich der LEH mittlerweile durch eine hohe Nachfragemacht gegenüber seinen Produzenten aus (Nielsen Tradedimensions, 2019 aus Fleischwirtschaft; Sexton \& Xia, 2018), die es ihm ermöglicht auch Einfluss auf die Produktionsausrichtung landwirtschaftlicher Betriebe zu nehmen.

Ein Beispiel für die zunehmend aktivere Rolle des LEHs ist die schrittweise Auslistung von Käfigeiern beginnend im Jahr 2003, die zu einer EU-weiten Anpassung der gesetzlichen Rahmenbedingungen geführt hat. Ein weiteres Beispiel ist die 2019 vom LEH eigenständig eingeführte Haltungskennzeichnung für Frischfleisch. Anhand von vier Stufen (Stallhaltung, Stallhaltung plus, Außenklima, Premium) können Verbraucher den Tierwohlstandard der 
Haltungsbedingungen identifizieren und besser informierte Konsumentscheidungen fällen. Bei diesem Standard kennzeichnet die erste Stufe den gesetzlichen Standard und die letzte u.a. biologisch produziertes Fleisch. Unklarheit besteht aktuell hinsichtlich zentraler Treiber und Hemmnisse von Lebensmitteleinzelhändlern, die auf eine Investition in alternative Vermarktungskonzepte wirken. Das Wissen darum ist jedoch von Bedeutung, um die Gestaltungsmöglichkeiten des LEHs in der Transformation der Nutztierhaltung besser zu verstehen.

\section{Aufbau der Dissertation}

Die vorliegende Dissertation besteht aus drei Kapiteln. Kapitel 1 untersucht zunächst die gesellschaftliche Wahrnehmung heimlich gemachter Aufnahmen aus landwirtschaftlichen Ställen. Dabei werden Treiber der gesellschaftlichen Meinungsbildung sowie die Umsetzung einer verschärften strafrechtlichen Ahndung teilweise gesetzeswidriger Protestformen beleuchtet. Zudem werden Unterschiede zwischen verschiedenen Ausprägungen heimlicher Aufnahmen untersucht. Ein weiterer Teil der Arbeit beschäftigt sich mit Konsumentenpräferenzen und Zahlungsbereitschaften für nachhaltig produzierte Fleischwaren, anhand von Rindfleisch aus Weidehaltung. Danach werden die Motive und Hemmnisse des LEHs Konsum- und Produktionsmuster nachhaltig zu beeinflussen, ebenfalls am Beispiel der Vermarktung von Rindfleisch aus Weidehaltung, näher betrachtet. Mit diesen drei Themenkomplexen trägt die Dissertation dazu bei, ein tieferes Verständnis für den Transformationsprozess der Nutztierhaltung, geprägt von gesellschaftlichen Anforderungen, Konsumpräferenzen und Gestaltungsmöglichkeiten des LEHs, zu entwickeln. Einen genauen Überblick über die vorliegende kumulative Dissertation sowie die darin enthaltenden Studien gibt Tabelle 1 . 
Tabelle 1. Aufbau der Dissertation

Kapitel Manuskripte

\section{Einleitung}

Teil I - Gesellschaftliche

Wahrnehmung heimlicher

Stallaufnahmen
I.1 Heimliche Stallaufnahmen aus gesellschaftlicher Sicht im Wechselspiel zwischen Landwirtschaft,

Tierschutzorganisationen und staatlichen Kontrollmechanismen

I.2 Public Perceptions of Undercover Investigations in Livestock Farming: An End that Justifies the Means?

II.1 Do consumers prefer pasture-raised dual-purpose cattle when considering meat products? A hypothetical discrete choice experiment for the case of minced beef

III.1 Food Retailers as Mediating Gatekeepers between Farmers and Consumers in the Supply Chain of Animal Welfare Meat - Studying Retailers' Motives in Marketing Pasture-Based Beef

III.2 Challenges and Motives in Food Retail Cooperatives' Approach to Supporting Alternative Niche Paths Using the Case of Locally Produced Grass-Fed Beef

Resümee und Ausblick

\section{Teil I - Gesellschaftliche Wahrnehmung heimlicher Stallaufnahmen}

Der erste Teil der Arbeit befasst sich mit der gesellschaftlichen Wahrnehmung neuerer teilweise gesetzeswidriger - Protestformen gegen die moderne Nutztierhaltung. Konkret wird in diesem Zusammenhang die gesellschaftliche Zustimmung und Legitimität heimlicher Aufnahmen betrachtet, die durch unbefugtes Eindringen in landwirtschaftliche Ställe von Tierschützern entstehen und über die Medien verbreitet werden (z.B. Associated Press, 2017; Deter, 2017; FAKT, 2019). Die investigativen Aufnahmen können einerseits dazu beitragen gesetzeskonforme, aber gesellschaftlich nur wenig bekannte, Produktionsverfahren sichtbar zu machen und andererseits konkrete Tierschutzvergehen in Haltung, Transport oder Schlachtung aufzudecken. Ersteres kann gesellschaftliche Diskussionen über gängige Produktionsverfahren anstoßen (z.B. Sauenhaltung in Kastenständen) und Druck auf Anpassung der bisherigen Haltungsverfahren ausüben. Zweiteres kann dazu führen sog. ,schwarze Schafe' und damit Tierschutzvergehen aufzudecken und zu unterbinden (z.B. Fall Straathof). Ob die Gesellschaft heimliche Aufnahmen aufgrund ihrer Bedeutung für Tierschutz und gesellschaftlicher Aufklärung befürwortet, obwohl sich diese in einer zivil- und strafrechtlichen Grauzone befinden, und $\mathrm{ob}$ es Unterschiede zwischen verschiedenen Ausprägungen heimlicher Aufnahmen gibt, ist bisher nicht abschließend geklärt. 
Im ersten Beitrag „Heimliche Stallaufnahmen aus gesellschaftlicher Sicht im Wechselspiel zwischen Landwirtschaft, Tierschutzorganisationen und staatlichen Kontrollmechanismen“ werden daher zunächst Treiber der gesellschaftlichen Wahrnehmung heimlicher Aufnahmen und ihrer strafrechtlichen Ahndung identifiziert.

Da heimliche Aufnahmen je nach Schwere des aufgedeckten Tierschutzvergehens (gesetzeskonforme Bedingungen vs. Tierschutzvergehen) sowie der Art des verschafften Zugangs (Blick durch offene Stalltür vs. Aufbrechen der Stalltür) variieren (Associated Press, 2017; FAKT 2019; Lenfers, 2019) betrachtet der zweite Beitrag mit dem Titel „Public Perceptions of Undercover Investigations in Livestock Farming: An End that Justifies the Means?“ die gesellschaftliche Legitimität und die gesellschaftliche Einordnung der Notwendigkeit einer strafrechtlichen Ahnung.

Damit kann der erste Teil der vorliegenden Dissertation gesellschaftliche Anforderungen an systemische Anpassungen identifizieren, die dazu führen, dass teilweise illegale Protestformen gesellschaftliche $\mathrm{Zu}-$ bzw. Ablehnung finden.

\section{Teil II - Konsumentenpräferenzen und Zahlungsbereitschaften für nachhaltig erzeugte Fleischwaren}

Da eine Transformation hin $\mathrm{zu}$ höheren Tierwohlstandards unweigerlich mit höheren Produktionskosten einhergeht (Clark et al., 2017), müssen gesellschaftliche Erwartungen in individuelle Konsumentscheidungen umgewandelt werden. Aus diesem Grund untersucht der zweite Teil der vorliegenden Dissertation Konsumentenpräferenzen und Zahlungsbereitschaften für alternativ erzeugte Fleischwaren. Die gesellschaftlichen Anforderungen an eine tierfreundliche Tierhaltung lassen sich vor allem in weniger intensiv gehaltenen Produktionssystemen, wie z.B. der Weidehaltung wiederfinden, denn Weidehaltung ermöglicht das Ausleben natürlicher Verhaltensweisen wie z.B. Bewegungsfreiheit, soziale Interaktion mit Artgenossen sowie das Erleben verschiedener klimatischer Einflüsse. Zusätzlich zeichnet sich insbesondere die extensive Weidehaltung durch Vorteile in Bezug auf Umwelt- und Naturschutz aus (Koncz et al., 2019; Morgan-Davies et al., 2014). In der Fleischvermarktung spielt Weidehaltung trotz des scheinbar großen Marktpotenzials bisher aber eine untergeordnete Rolle.

Am Beispiel von Weiderindfleisch wird daher im Beitrag „Do consumers prefer pasture-raised dual-purpose cattle when considering meat products? A hypothetical discrete choice 
experiment for the case of minced beef" untersucht, welche Produkteigenschaften für den Konsumenten in der Kaufentscheidung von Relevanz sind.

Damit kann der zweite Teil dieser Dissertation in der Kaufentscheidung relevante, nachhaltige Produktattribute identifizieren, die erfolgsversprechende Differenzierungsmöglichkeiten am Markt für Frischfleisch darlegen.

\section{Teil III - Der Lebensmitteleinzelhandel als Gestalter nachhaltiger Konsum- und Produktionsmuster}

Im Rahmen des dritten Teils der vorliegenden Dissertation mit dem Titel „Der Lebensmitteleinzelhandel als Gestalter nachhaltiger Konsum- und Produktionsmuster"wird der LEH näher untersucht. Der moderne LEH ist durch Globalisierung, Effizienzsteigerungen, Standardisierung sowie hohe Marktkonzentrationen gekennzeichnet. Die größten fünf deutschen Unternehmen im LEH (Edeka, Rewe, Schwarz, Aldi \& Metro) erzielen einen Marktanteil von über 75\% (Nielsen Tradedimensions, 2019 aus Fleischwirtschaft) und zeichnen sich dadurch durch eine hohe Marktmacht aus. Frischfleischwaren werden im LEH gewöhnlich zu einem möglichst geringen Preis angeboten. Eine Differenzierung nach Qualitätsmerkmalen, wie einem erhöhten Tier- oder Umweltschutz in der Produktion sind wenig verbreitet und gewinnen erst langsam an Bedeutung. Als wichtigster Einkaufsort für Fleisch in Deutschland (Kohlmüller \& Koch, 2018) kann dem hochkonzentrierten LEH aber eine vielversprechende Rolle im Transformationsprozess der modernen Nutztierhaltung zugesprochen werden. Als ,ökologischer Türöffner' kann er den Erfolg nachhaltiger Fleischwaren beeinflussen, in dem er entscheidet welchen Produkten er Zugang zum Massenmarkt gewährt und welchen nicht (Hansen, 1993). In jüngster Zeit hat der Handel zunehmend neue Vermarktungswege etabliert, die dem bestehenden Wettbewerbsmodell des Fleischsektors (Kostenführerschaft) teilweise widersprechen und qualitativ hochwertige und nachhaltig erzeugte Fleischwaren fördern (Bui et al., 2019). Da die Vermarktung von möglichst preiswertem Frischfleisch für den LEH nach wie vor ein wichtiger Bestandteil in seinen Marketingmaßnahmen ist (Verbreitung von sog. ,Schweinebauchanzeigen'), ist die Listung nachhaltiger Waren, die durch höhere Produktpreise gekennzeichnet sind, mit unternehmerischen Unsicherheiten verbunden.

Um zu verstehen warum einige Lebensmitteleinzelhändler trotzdem Fleischwaren mit höheren Tierwohlstandards listen, werden im ersten Beitrag mit dem Titel „Food retailers as mediating gatekeepers between farmers and consumers in the supply chain of animal welfare meatstudying retailers ' motives in marketing pasture-based beef" am Beispiel der Vermarktung von Weiderindfleisch, die Motive der Listungsentscheidungen näher untersucht. 
In jüngster Zeit sind zudem Initiativen des, vor allem genossenschaftlich organisierten, LEHs $\mathrm{zu}$ beobachten, in die Zusammenarbeit mit regionalen Landwirten zu investieren (z.B. 'Unsere Region - Gutes von Hier' der Edeka Gruppe, 'Aus deiner Region' der Rewe Group). Damit gehen sie einen alternativen, dezentralisierten Vermarktungsweg, der lokale landwirtschaftliche Betriebe fördert. Diese Betriebe können oftmals den standardisierten Anforderungen des LEHs nicht gerecht werden, produzieren aber alternative Lebensmittel produzieren, die einen Beitrag zu einer nachhaltigeren landwirtschaftlichen Produktion leisten können. Der zweite Beitrag mit dem Titel „Challenges and motives in food retailers' approach to support alternative niche paths- using the case of locally produced grass-fed beef" untersucht, ebenfalls am Beispiel von Weiderindfleisch, mit welchen Herausforderungen der genossenschaftlich organisierte Einzelhandel bei der Zusammenarbeit mit regionalen Landwirten konfrontiert ist. Außerdem wird analysiert, was Lebensmitteleinzelhändler trotzdem dazu motiviert einen Mehraufwand, der unweigerlich mit der Abkehr des bisher stark von Standardisierung und Zentralisierung geprägten Vermarktung, verbunden ist, auf sich zu nehmen.

Damit trägt der dritte Beitrag der vorliegenden Dissertation zu einem besseren Verständnis der Rolle des LEHs im Transformationsprozess bei. Wissen über die Herausforderungen und Motive der Listungsentscheidungen kann dazu beitragen die Möglichkeiten und Herausforderungen des LEH zu fördern bzw. zu minimieren. Die Dissertation schließt mit einem Resümee und Ausblick. 


\section{Literatur}

Associated Press November (2017). Undercover video shows shocking cow abuse. New York Post online. https://nypost.com/2017/11/10/undercover-video-shows-shocking-cowabuse/.

BBV (Bayerischer Bauernverband) (2016). Pressemitteilung - Stalleinbruch: Aufruhr im Putenstall. https://www.bayerischerbauernverband.de/pm60-stalleinbruch.

BMEL (Bundesministerium für Ernährung und Landwirtschaft) (2019). Statistisches Jahrbuch über Ernährung, Landwirtschaft und Forsten. Berlin. https://www.bmelstatistik.de/archiv/statistisches-jahrbuch/.

Bodderas, E. \& Graw, A. (2019). 200.000 männliche Kälber werden jährlich illegal getötet. Welt Wirtschaft. https://www.welt.de/wirtschaft/article203765326/200-000maennliche-Kaelber-werden-jaehrlich-illegal-getoetet.html.

Boogaard, B. K., Bock, B.B., Oosting, S. J., Wiskerke, J. S. C. \& van der Zijpp, A. J. (2011). Social Acceptance of Dairy Farming. The Ambivalence Between the Two Faces of Modernity. Journal of Agricultural and Environmental Ethics, 24(3), 259-282.

Borkfelt, S., Kondrup, S., Röcklinsberg, H., Bjørksahl, K. \& Gjerris, M. (2015). Closer to Nature? A Critical Discussion of the Marketing of "ethical" animal products. Journal of Agricultural and Environmental Ethics, 28(6), 1053-1073.

Bui, S., Costa, I., De Schutter, O., Dedeurwaerdere, T., Hudon, M., \& Feyereisen, M. (2019). Systemic ethics and inclusive governance: Two key prerequisites for sustainability transitions of agri-food systems [Special issue]. Agriculture and Human Values, 36(2), 277-288.

Clark, B., Stewart, G.B., Panzone, L.A., Kyriazakis, I. \& Frewer, L.J. (2017). Citizens, consumer and farm animal welfare: A meta-analysis of willingness-to-pay studies. Food Poilicy, 68 (April 2017), 112-127.

Deter, A. (2017). OLG bestätigt Rechtmäßigkeit von Stalleinstieg durch Tierrechtler.

Topagrar online. https://www.topagrar.com/news/Home-top-News-Gericht-bestaetigtRechtmaessigkeit-von-Stalleinstieg-durch-Tierrechtler-9056491.html.

Fakt (2019). Milchviehskandal in Bayern - Mangelt es an Kontrollen? ARD Mediathek. https://www.ardmediathek.de/daserste/player/Y3JpZDovL21kci5kZS9iZW10cmFnL2 Ntcy9mMWQzY2ExMS03NWQ5LTRjMjEtOTBiNi00ZWY1ZjgwNDRmNmM/milc hviehskandal-in-bayern-mangelt-es-an-kontrollen.

Geels, F. W. \& Schot, J. (2007). Typology of sociotechnical transition pathways. Research Policy, 36(3), 399-417. 
Gilbert, D. (2003). Retail Marketing Management. Second edition. Financial Times/ Prentice Hall, Harlow.

Godfray, H.C.J. \& Garnett, T. (2014). Food security and sustainable intensification. Philosophical Transactions of the Royal Society B, 369, 20120273.

Hansen, U. (1993). Ökologisches Marketing im Handel. In: E. Seidel, H. Strebel, ed. Betriebliche Umweltökonomie, 448-479. Wiesbaden: Gabler.

Hölker, S., Steinfath, H., Meyer-Höfer, M.v. \& Spiller, A. (2019). Tierethische Intuitionen in Deutschland: Entwicklung eines Messinstrumentes zur Erfassung bereichsspezifischer Werte im Kontext der Mensch-Tier-Beziehung. German Journal of Agricultural Economics, 69(4), 299-315.

Johnson, J. (2014). On the ethics of ag-gag legislation and undercover investigations. Northern Plains Ethics Journal, (Fall 2014), 30-39.

Kayser, M., Böhm, J. \& Spiller, A. (2011). Die Agrar- und Ernährungswirtschaft in der Öffentlichkeit -Eine Analyse der deutschen Qualitätspresse auf Basis der FramingTheorie, Yearbook of Socioeconomics in Agriculture, 4(1), 59-83.

Koalitionsvertrag Deutschland (2018). https://www.cdu.de/system/tdf/media/dokumente /koalitionsvertrag_2018.pdf?file=1.

Koeck, A., Loker, S., Miglior, F., Kelton, D.F., Jamrozik, J. \& Schenkel, F.S. (2014). Genetic relationships of clinical mastitis, cystic ovaries, and lameness with milk yield and somatic cell score in first-lactation Canadian Holsteins. Journal of Dairy Science, 97(9), 5806-5813.

Koehler, J., Geels, F.W., Kern, F., Markard, J., Onsongo, E., Wieczorek, A., Alkemade, F. ,... \& Wells, P. (2019). An agenda for sustainability transitions research: State of the art and future directions. Environmental Innovation and Societal Transitions, 31(June 2019), 1-32.

Kohlmüller, M. \& Koch. T. (2018). Markt Bilanz - Vieh und Fleisch, ed. AMI (Argrarmarkt Informationsgesellschaft). Bonn: Medienhaus Plump GmbH.

Koncz, N.K., Béri, B. Deák, B., Kelemen, A., Tóth, K., Kiss, R., Radócz, S., Miglécs, T., Tóthmérész, B., \& Valkó, O. (2019). Meat production and maintaining biodiversity: Grazing by traditional breeds and crossbred beef cattle in marshes and grasslands. Applied Vegetation Science, 23(2), 139- 148.

Krüsken, B. (2017). Stalleinbrüche rechtlich ächten. Grüner Dienst, Deutscher Bauernverband, Ausgabe vom 17.08.2017, KW 33. 
Krüsken, B. (2017). Stalleinbrüche rechtlich ächten. Grüner Dienst, Deutscher Bauernverband, Ausgabe vom 17.08.2017, KW 33.

Lagerkvist, C. J., Carlsson, F. \& Viske, D. (2006). Swedish Consumer Preferences for Animal Welfare and Biotech: A Choice Experiment. AgBioForum, 9(1), 51-58.

Lenfers, C. (2019). Fall Röring: Alarmanlage und Lüftung manipuliert. Topagrar online. https://www.topagrar.com/schwein/news/fall-roering-alarmanlage-und-lueftungmanipuliert-10152315.html.

Moewius, J. P., Röhring, D. Schaack, C., Ramphold, H. J., Brzukalle, F.G., Stein-Bachinger, S., Wolter, M. \& Sanders, J. (2018). Zahlen, Daten, Fakten - Bio-Branche 2018, BÖLW (Bund Ökologischer Lebensmittelwirtschaft e.V.), ed., Berlin: Spree Print.

Morgan-Davies, J., Morgan Davies, C., Pollock, M.L., Holland, J.P. \& Waterhouse, A. (2014). Characterisation of extensive beef cattle systems: Disparities between opinions, practice and policy. Land Use Policy, 38 (May 2014), 707-718.

Nielsen Trade Dimensions (2019). Top-Firmen Edition 2019. Aus: Fleischwirtschaft.de (2019). Die Top 5 bleiben an der Spitze.

https://www.fleischwirtschaft.de/wirtschaft/charts/Lebensmittelhandel-Die-Top-5bleiben-an-der-Spitze-40682.

Peattie, K. \& Peattie, S. (2009). Social marketing: a pathway to consumption reduction? Journal of Business Research, 62(2), 260-268.

Pirsich,W., von Hardenberg, L. \& Theuvsen, L. (2017). Eine empirische Analyse zum Angebot von Tierwohl-Fleisch in Fleischerfachgeschäften. Berichte über Landwirtschaft, 95(2), 1-28.

Robbins, J. A., Weary, D. M., Schuppli, C. A. \& von Keyserlingk, M. A.G. (2015). Stakeholder views on treating pain due to dehorning dairy calves. Animal Welfare, 24(4), 399-406.

Rojas-Downing, M.M, Nejadhashemi, A.P., Harrigan, T. \& Woznicki, S.A. (2017). Climate change and livestock: Impacts, adaption and mitigation. Climate Risk Management, 16(2017), 145-163.

Rouha-Mülleder, C., Palme, R. \& Waiblinger, S. (2010). Erhebungen zur Tiergerechtheit in 80 Milchkuhbetrieben mit Boxenlaufställen - Tiergesundheit und andere tierbezogene Indikatoren. Wiener Tierärtzliche Monatsschrift, 97, 231-241.

Sanchez-Sabate, R.\& Sabaté, J. (2019). Consumer attitudes towards environmental concern of meat consumption: A Systematic Review. International Journal of Environmental Research and Public Health, 16(1229), 1-37. 
Sandøe, P., Corr, S., Palmer, C. \& Bonnet, B. (2016). Selective breeding. In: P. Sandøe., S. Corr \& C. Palmer, ed. Companion animal ethics. Chichester, UK: John Wiley and Sons (UFAW animal welfare series).

Sands, S., Oppewal, H. \& Beverland, M. (2009). The effects of in-store themed events on consumer choice decisions. Journal of Retailing and Consumer Services, 16(5), 386395 .

Schlecht, S., Albersmeier, F. \& Spiller, A. (2010). Eine Analyse medialer Frames bei Konflikten im ländlichen Raum -das Beispiel landwirtschaftlicher Bauvorhaben, in: Kayser, M., Böhm, J., Spiller, A. ed. Die Ernährungswirtschaft in der Öffentlichkeit. Social Media als neue Herausforderung der PR, Göttingen: Cuvillier Verlag: 333-364.

Schneider, L., Kemper, N. \& Spindler, B. (2020). Stereotypic Behavior in Fattening Bulls. Animals, 10(40), 1-9.

Sexton, R. \& Xia, T. (2018). Increasing Concentration in the Agricultural Supply Chain: Implications for Market Power and Sector Performance. Annual Review of Ressource Economics, 20(2018), 229-251.

Spooner, J. M., Schuppli, C. A. \& Fraser, D. (2014). Attitudes of Canadian Citizens Toward Farm Animal Welfare: A Qualitative Study. Livestock Science, 163(May 2014), 150158.

Statistisches Bundesamt (2010). Landwirtschaftszählung/Agrarstrukturerhebung 2010. Fachserie 3, Heft 6. https://www.destatis.de/DE/Themen/Branchen-Unternehmen/ Landwirtschaft-Forstwirtschaft-Fischerei/Produktionsmethoden/ Publikationen/ Downloads-Produktionsmethoden/stallhaltung-weidehaltung-2032806109004.pdf

Tonsor, G.T. \& Olynk, N.J. (2010). U.S. Meat Demand: The Influence of Animal Welfare Media Coverage. Agricultural Experiment Station and Cooperative Extension Service, Kansas State University, Manhatten.

Vanhonacker, F. \& Verbeke, W. (2014). Public and consumer policies for higher welfare food products: Challenges and Opportunities. Journal of Agricultural and Environmental Ethics, 27(1), 153-171.

Vizzier Thaxton, Y., Christensen, K. D., Mench, J.A..; Rumley, E. R.; Daugherty, C., Feinberg, B., Parker, M., ... \& Scanes, C.G. (2016). Symposium: Animal welfare challenges for today and tomorrow. Poultry Science, 95(9), 2198-2207.

Zander, K., Bürgelt, D., Christoph-Schulz, I., Salamon, P. \& Weible, D. (2013). Erwartungen der Gesellschaft an die Landwirtschaft. Abschlussbericht: Stiftung Westfälische Landschaft. https://literatur.thuenen.de/digbib_extern/dn052711.pdf. 


\section{Teil I - Gesellschaftliche Wahrnehmung heimlicher Stallaufnahmen}

I.1 Heimliche Stallaufnahmen aus gesellschaftlicher Sicht im Wechselspiel zwischen Landwirtschaft, Tierschutzorganisationen und staatlichen Kontrollmechanismen

Autoren: Maureen Schulze, Antje Risius, Achim Spiller

Dieser Beitrag ist erschienen im German Journal of Agricultural Economics 2018, 67(4), 267-280. 


\section{Zusammenfassung}

Heimliche Aufnahmen aus landwirtschaftlichen Ställen haben in den letzten Jahren große mediale Aufmerksamkeit erlangt. Tierschutzorganisationen, Landwirte und Politik diskutieren über die Auswirkungen heimlicher Aufnahmen sowie die Konsequenzen für Tierschützer, die heimlich in Ställe eindringen, um dort die Bedingungen zu filmen. Die Einstellung der Gesellschaft $\mathrm{zu}$ Undercover-Videos in landwirtschaftlichen Ställen hat bisher wenig wissenschaftliche Beachtung gefunden und wird daher im Rahmen dieser explorativen Erhebung mit 292 Befragten beleuchtet. Mithilfe einer Strukturgleichungsmodellierung werden Einstellungen der Gesellschaft auf die Akzeptanz von heimlichen Aufnahmen analysiert. Es zeigt sich, dass heimliche Aufnahmen aus Tierschutzgründen überwiegend als notwendig angesehen werden, weil staatliche Kontrollmechanismen nicht ausreichen, die Arbeit der verantwortlichen Tierschutzorganisationen gesellschaftliche Zustimmung erfährt und Unsicherheit bezüglich der psychischen Belastung betroffener Landwirte besteht. Beeinflusst wird die positive Grundeinstellung gegenüber heimlichen Aufnahmen durch die zunehmend kritische Betrachtung der landwirtschaftlichen Tierhaltung. Eine härtere strafrechtliche Ahndung findet wenig gesellschaftlichen Zuspruch. Heimliche Aufnahmen zeigen einmal mehr die Diskrepanz zwischen Landwirtschaft und Gesellschaft auf und verdeutlichen, dass das Wohlergehen landwirtschaftlicher Nutztiere vermehrt in den Fokus rückt. 


\begin{abstract}
Undercover investigations in animal husbandry have attracted great media attention in recent years. Animal welfare organizations, farmers and politicians have been discussing the need for undercover investigations and their consequences for animal activists. Since the public perspective and its influencing factors had not previously been scientifically investigated an exploratory study with 292 German consumers is conducted. Using structural equation modeling the attitude to undercover investigations and their influencing factors are revealed. Generally, the results show a positive attitude towards undercover investigations. They are perceived as necessary for animal welfare reasons because state-owned control mechanisms are unsatisfactory. In addition, the results show that the work of responsible animal welfare organizations receives social approval and the public is unsure about the psychological burden on affected farmers. The positive attitude towards undercover investigations is influenced by the increasingly critical view on livestock farming. However, the public rejects harsher punishment of investigators. Undercover investigations demonstrate the discrepancy between agriculture and society and show that the welfare of farm animals is becoming increasingly important.
\end{abstract}




\section{Einleitung}

Möglicherweise kranke und vernachlässigte Schweine, die sich nicht einmal umdrehen können, eng gedrängtes Geflügel mit lückenhaftem Gefieder und blutiger Haut: Diese und ähnliche heimlich gedrehte Aufnahmen aus Ställen begegnen den Verbrauchern immer wieder (u.a. Frontal 21 vom 13.03.2018; Frontal 21 vom 28.03.2018). Die Landwirtschaft ist empört. Tiere würden gefährdet, Sachbeschädigungen verübt und nicht zuletzt wird auch die Angst vor öffentlicher Bloßstellung genannt (TONSOR und OLYNK, 2010; BBV, 2016; KRÜSKEN, 2017). Im Gegensatz dazu sehen Nichtregierungsorganisationen (NGOs) heimliche Aufnahmen als wichtiges Hilfsmittel um Tierschutzvergehen aufzudecken (JOHNSON, 2014). Dass dabei ggf. strafrechtliche und zivilrechtliche Grenzen überschritten werden, hindert sie nicht daran, sich unerlaubt Zugang zu landwirtschaftlichen Ställen zu verschaffen (DETER, 2016; DETER, 2017). Die heimlichen Aufnahmen werden i.d.R. über Medien verbreitet, um die Gesellschaft mit den dokumentierten Haltungsbedingungen zu konfrontieren. Die gesellschaftliche Einstellung zu heimlichen Aufnahmen hat bisher allerdings kaum Beachtung gefunden. Dies gilt sowohl für die mediale Diskussion als auch für wissenschaftliche Untersuchungen. Unklar ist dabei auch die gesellschaftliche Einstellung zu einer verschärften strafrechtlichen Ahndung.

In der Vergangenheit konnte bereits gezeigt werden, dass die gesellschaftliche Beurteilung die Akzeptanz oder Ablehnung von Haltungsbedingungen maßgeblich beeinflussen kann und aufkommende gesellschaftliche Bedenken zu Veränderungen von gesetzlichen Vorschriften führen können (SPILLER ET AL., 2016; SonNTAG und SPILlER, 2016). Sollten heimliche Aufnahmen gesellschaftlich als Hilfsmittel zur Kontrolle der Haltungsbedingungen akzeptiert werden, vertieft dies möglicherweise die ohnehin konfliktäre Auseinandersetzung zwischen Landwirtschaft und Gesellschaft (z.B. SCHLECHT et al., 2010). Ziel der Studie ist eine erste Einschätzung der gesellschaftllichen Bewertung solcher Aufnahmen sowie der sich daraus ergebenden Einstellung zur Einführung eines Gesetzes, das heimliche Aufnahmen unter Strafe stellt. Damit sollen die Hintergründe eines sowohl für die Landwirtschaft als auch für die Zivilgesellschaft wichtigen Themas empirisch beleuchtet werden.

\section{Literaturüberblick}

\section{Rechtliche Situation}

In Deutschland existieren keine spezifischen strafrechtlichen Normen, die heimliche Aufnahmen verbieten oder sie rechtfertigen. Im allgemeinen Strafrecht kommen je nach 
Sachverhalt Verurteilungen unter anderem wegen Sachbeschädigung und Hausfriedensbruch auf der Seite der Aktivisten in Betracht. Kommt es zu Auseinandersetzungen mit den betroffenen Landwirten, können auch Verurteilungen wegen Körperverletzung oder Nötigung sowohl auf der Seite der Aktivisten als auch auf Seiten der Landwirte relevant werden. Zusätzlich können Aktivisten, aber auch Landwirte aufgrund von Tierschutzverstößen zur Rechenschaft gezogen werden. Derzeit wird anhand der bisherigen Entscheidungen im strafrechtlichen Bereich deutlich, dass eine gesetzeskonforme Tierhaltung eher zu einer Verurteilung der Aktivisten führt und in Fällen, in denen die Haltungsbedingungen nicht den gesetzlichen Vorgaben entsprechen, eher von einer Verurteilung abgesehen wird (OBerlandesgericht NAumburg, Urt. v. 22.02.2018, Az. 2 Rv 157/17). Es können auch zivilrechtliche Schadensersatzansprüche ausgelöst werden, wenn das Eigentum des Landwirtes beschädigt wurde (HIRT et al., 2016). Werden darüber hinaus heimliche Film- oder Bildaufnahmen veröffentlicht, spielen Fragen des Medienrechts eine Rolle. Hierbei kommt der Abwägung zwischen dem Recht auf freie Meinungsäußerung und Informationen auf der einen Seite und dem Recht am eigenen Bild sowie dem Schutz der persönlichen Daten auf der anderen Seite eine wichtige Bedeutung zu. Insgesamt geht es im Rahmen der rechtlichen Gesamtabwägung darum, Tierschutz zu gewährleisten, den Rechten des betroffenen Landwirtes gerecht $\mathrm{zu}$ werden und die Allgemeinheit über wesentliche Fragen im Bereich der Nutztierhaltung zu informieren.

Gesellschaftspolitisch ist vor allem die Einführung einer strafrechtlichen Gesetzesnorm umstritten. In Deutschland spricht sich die Landwirtschaft für eine härtere Bestrafung heimlicher Aufnahmen aus. Es wird gefordert, heimliche Aufnahmen mit Diebstahlsverstößen und Wohnungseinbrüchen gleichzusetzen (KRÜSKEN, 2017). Auch einige Politiker sehen heimliche Aufnahmen nicht als geeignetes Hilfsmittel, Tierrechtsverstöße aufzudecken und sprechen sich ebenfalls für eine härtere Bestrafung der Aktivisten aus (Awater-Esper, 2017). In den USA ist die gesellschaftspolitische Diskussion um heimliche Aufnahmen schon weiter fortgeschritten (KOEHLER, 2013; ROBBINS et al., 2016). Auf zahlreiche verdeckte Aufnahmen (ANIMAL VISUALS, 2015) reagieren einige Bundesstaaten mit konkreten Strafgesetznormen, die Aufnahmen aus landwirtschaftlichen Ställen ohne Genehmigung des Landwirts unter Strafe stellen (Shea, 2014). Dies gilt unter anderem auch für ein unter falschem Vorwand entstandenes Arbeitsverhältnisses, welches ausschließlich zum Zwecke der Dokumentation von Haltungsbedingungen in landwirtschaftlichen Ställen - als Form des Whistleblowings eingegangen wurde (MARCUEA, 2015). 


\section{Gesellschaftliche Positionen zu heimlichen Aufnahmen und einer stärkeren rechtlichen Strafandrohung}

Die Diskussion zwischen Tierschutzorganisationen, Landwirten, der Gesellschaft und Politik um heimliche Aufnahmen ist vielschichtig. Nach Aussagen von Tierrechtsorganisationen, welche die heimlichen Stallaufnahmen verteidigen, können durch versteckte Aufnahmen nicht gesetzeskonforme Produktionsweisen sowie ein unsachgemäßer Umgang mit Nutztieren wirkungsvoll aufgedeckt werden (JOHNSON, 2014). Begründet wird diese Aussage vor allem durch fehlende oder nicht wirksame Kontrollmechanismen, die nicht ausreichend in der Lage sind, Missstände aufzudecken (DETER, 2013). Heimliche Aufnahmen gelten als Hilfsmittel, das Wohlergehen und die Haltungsbedingungen von Nutztieren zu verbessern (STEPHAN, 2017). Außerdem scheinen heimliche Aufnahmen geeignet, Produktionsabläufe und -techniken sichtbar zu machen, die der Öffentlichkeit bisher nicht bekannt sind (JOHNSON, 2014). Dies kann zu einem gesellschaftlichen Akzeptanzverlust von gesetzeskonformen Produktionspraktiken führen. Folgen daraufhin Gesetzesänderungen, wie von SPILLER et al. (2016) beschrieben, können heimliche Aufnahmen zu einer generellen Veränderung von Haltungsbedingungen in der modernen Nutztierhaltung führen.

Gegner heimlicher Stallaufnahmen weisen auf eine mögliche Inszenierung von besonders schwerwiegenden Fällen hin. Derartige Bilder würden nicht den Maßstäben der modernen Nutztierhaltung entsprechen und die gezeigten Bedingungen nicht auf das Gros der Betriebe zu übertragen sein (BBV, 2016). Es wird kritisiert, dass den Tieren während nächtlicher Filmaufnahmen Leid zugefügt wird, da nicht ausreichend auf die Bedürfnisse der Tiere eingegangen werde oder Tiere absichtlich verletzt werden (BBV, 2016). Außerdem wird hinterfragt, ob die Motivation der Aktivisten in der Behebung von Missständen begründet sei oder Spendenakquise im Vordergrund stehe (KRÜSKEN, 2017). Landwirte sehen sich mit der Angst, von Aktivisten ins Visier genommen zu werden, konfrontiert. Werden heimliche Aufnahmen aus dem eignen Stall veröffentlicht, können neben den finanziellen Einbußen insbesondere die psychischen Folgen durch die öffentliche Bloßstellung relevant sein (DETER, 2017).

In wissenschaftlichen Untersuchungen hat die Einstellung zu heimlichen Aufnahmen bisher kaum Beachtung gefunden. LIEBE und JAHNKE (2017) beschäftigen sich mit den Motiven von Tierrechtsaktivisten, sich in der Tierrechtsbewegung zu engagieren. Zentrales Motiv beim Engagement ist das Wohl der Tiere, was maßgeblich dadurch beeinflusst wird, dass diese als dem Menschen hinsichtlich ihrer Empfindung von Schmerzen als sehr ähnlich wahrgenommen werden. Insbesondere der als vornehmlich sehr wirtschaftlich motiviert wahrgenommene 
Umgang mit den Tieren in der Landwirtschaft wird kritisiert. Um das Wohl der Tiere zu gewährleisten, ist es aus Sicht der Tierschützer notwendig, sich persönlich dafür einzusetzen und selbst aktiv zu werden, denn rechtliche Vorgaben sind aus ihrer Sicht nicht ausreichend. Neben diesen eher emotionalen Motiven ist auch die Unzufriedenheit mit dem politischen System ein zentraler Treiber, um sich in der Tierrechtsbewegung zu engagieren (LIEBE und JAHNKE, 2017). Im Rahmen eines von LIEBE und JAHNKE (2017) durchgeführten Vignettenexperiments mussten Tierrechts- und Tierschutzaktivisten die Aktionen „Parolen an die Wand sprühen“, „heimlich Aufnahmen im Stall machen“ oder die „Tiere befreien“ bewerten. Es zeigt sich, dass vor allem das Filmen von Haltungsbedingungen zur Dokumentation und zur Aufklärung auf Akzeptanz trifft. In einer weiteren Untersuchung von LIEBE et al. (2017) wurde untersucht, wie die Bevölkerung auf illegale Protestformen reagiert. Die Vignetten bestanden erneut aus den Merkmalen Ort, Handlung und Konsequenz, die die gleichen Ausprägungen aufwiesen. Es zeigt sich, dass alle illegalen Proteste von Tierschützern auf gesellschaftliche Zustimmung stoßen. Besonders stark befürwortet werden heimliche Aufnahmen (LIEBE et al., 2017).

$\mathrm{Zu}$ einem ähnlichen Ergebnis kommen Meinungsumfragen aus den USA. Sie stellen fest, dass heimliche Aufnahmen mit dem Zweck, Tierschutzvergehen aufzudecken, gesellschaftliche Zustimmung erfahren (ASPCA, 2012). TIPLADY et al. (2013, 2015) konzentrieren sich auf den gesellschaftlichen Umgang mit heimlichen Aufnahmen in Australien und arbeiten heraus, dass eine stark emotionale Reaktion auf tierschutzwidrige Aufnahmen zu erkennen ist, heimliche Aufnahmen zum Zwecke einer Aufdeckung von Tierschutzverstößen akzeptabel sind und trotz der emotionalen Betroffenheit der Wunsch nach weiteren Informationen erhalten bleibt.

Neben diesen generellen Einstellungsstudien existiert eine wissenschaftliche Untersuchung, die sich auf die gesellschaftliche Einstellung zu verschärften Gesetzen in den USA konzentriert. RoBbINS et al. (2016) beschäftigen sich mit der Frage, inwiefern Kenntnisse über spezielle Gesetze, die heimliche Aufnahmen bestrafen, einen Einfluss auf das gesellschaftliche Vertrauen in die Landwirtschaft haben. Sie verdeutlichen mit ihren Ergebnissen, dass ein durch Gesetze hervorgebrachter eingeschränkter Zugang zu Informationen den Vertrauensverlust in die Landwirtschaft fördert. Diese Ergebnisse bestätigen sich auch in anderen Meinungsumfragen aus den USA. Gesetze, die heimliche Aufnahmen bestrafen, werden laut Meinungsumfragen von großen Teilen der amerikanischen Gesellschaft abgelehnt (ASPCA, 2012), obwohl sie, wie bereits ausgeführt, in einigen Bundesstaaten eingeführt sind (SHEA, 2014). 


\section{Hypothesen}

Bereits in der Vergangenheit hat sich gezeigt, dass gesellschaftliche Kritik zu Veränderungen der gesetzlichen Standards in der Tierhaltung führen kann (z.B. Verbot von Käfighaltung bei Legehennen) (SPILLER et al., 2016). Es kann daher angenommen werden, dass sich aus der gesellschaftlichen Einstellung zu heimlichen Aufnahmen die gesellschaftliche Einstellung zu einer härteren strafrechtlichen Ahndung ableitet. Aufgrund der in 2.2 dargestellten Ergebnisse wird angenommen, dass heimliche Aufnahmen gesellschaftliche Zustimmung erfahren (TIPLADY et al., 2013; TIPLADY et al., 2015; LIEBE et al., 2017) und eine strafrechtliche Verschärfung kaum gesellschaftlichen Anklang findet (ASPCA, 2012; RoBBINS et al., 2016).

Da es bisher kaum Analysen zu den Hintergründen der gesellschaftlichen Meinungsbildung zu heimlichen Aufnahmen gibt, können dazu im Folgenden nur vorsichtig erste Hypothesen formuliert werden. Es kann angenommen werden, dass die gesellschaftliche Einstellung zu heimlichen Aufnahmen in einem engen Zusammenhang mit der Einstellung zur landwirtschaftlichen Tierhaltung steht. Aus diesem Grund wird die Hypothese aufgestellt, dass eine zunehmende Unzufriedenheit mit der modernen Nutztierhaltung mit einer gesellschaftlichen Zustimmung heimlicher Aufnahmen einhergeht. Es wird angenommen, dass heimliche Aufnahmen gesellschaftlichen Anklang finden, wenn der Landwirtschaft eine reine gewinnorientierte Produktionsweise nachgesagt wird (LIEBE und JAHNKE 2017; ZANDER et al., 2013) oder Haltungsbedingungen (LIEBE und JAHNKE, 2017) als nicht artgerecht wahrgenommen werden.

Weiterhin wird angenommen, dass das zunehmende gesellschaftliche Interesse am Wohlergehen landwirtschaftlicher Nutztiere (ZANDER et al., 2013; SPILLER et al., 2016) die Zustimmung zu heimlichen Aufnahmen fördert.

Aufgrund der in der medialen Diskussion immer wieder hinterfragten Glaubwürdigkeit heimlicher Aufnahmen und einer möglichen Inszenierung heimlicher Aufnahmen (z.B. BBV, 2016) wird außerdem angenommen, dass die Glaubwürdigkeit der Aufnahmen die gesellschaftliche Einstellung beeinflusst. Werden verdeckte Aufnahmen als glaubwürdig eingestuft, nimmt auch deren Zustimmung zu, so die Hypothese.

Basierend auf der Erkenntnis der gesellschaftlich geforderten Verstärkung von Kontrollen im Bereich der Lebensmittelproduktion (ZANDER et al., 2013) wird angenommen, dass ein geringes Vertrauen in bestehende Kontrollmechanismen mit einer gesellschaftlichen Befürwortung heimlicher Aufnahmen einhergeht.

Auf Basis bisheriger wissenschaftlicher Untersuchungen der Tierwohlforschung, aber auch Untersuchungen, die sich mit heimlichen Aufnahmen beschäftigen, wird weiterhin 
angenommen, dass zudem jüngere Menschen (MCKENDREE et al., 2014; Su und MARTENS, 2017), Menschen mit einem höheren Bildungsniveau (ZANDER et al., 2013), Frauen (MCKENDREE et al., 2014) und die eher städtisch wohnende Bevölkerung (ROBBINS et al., 2016) heimliche Aufnahmen befürworten. Auf Basis der Ergebnisse von RoBBINS et al. (2016) wird angenommen, dass Vegetarier heimliche Aufnahmen eher befürworten.

Weiterhin wird angenommen, dass Haustierbesitzer sich durch ein erhöhtes Tierwohlbewusstsein auszeichnen und daher heimliche Aufnahmen befürworten. Diese Hypothese wird zusätzlich durch Ergebnisse aus der Tierwohlforschung gestützt (MCKENDREE et al., 2014).

Die zunehmende gesellschaftliche Orientierung an Tierschutz- und Tierrechtsorganisationen bei der Suche nach tierwohlrelevanten Informationen (MCKENDREE et al., 2014) lässt einen starken Einfluss dieser Organisationen auf die gesellschaftliche Einstellung zu heimlichen Aufnahmen vermuten. Es wird daher angenommen, dass die Zugehörigkeit zu Tierschutz- und Tierrechtsorganisationen die gesellschaftliche Befürwortung heimlicher Aufnahmen fördert. Auch wenn bekannt ist, dass ein Unterschied zwischen Tierrecht und Tierschutz existiert (ROSCHER, 2012), werden im Rahmen dieser Untersuchung die Mitgliedschaften in Tierschutzund Tierrechtsorganisationen zusammengefasst.

Im Hinblick auf die Fragestellung dieser Untersuchung und auf Basis der bisher dargestellten Erkenntnisse und sachlogischen Überlegungen wurde abschließend das Untersuchungsmodell mit dargestellten Beziehungen (Abb. 1) entwickelt. 


\section{Abbildung 1. Modellspezifikation}

\section{Einstellung zur Landwirtschaft}

\begin{tabular}{|l|l|}
\hline $\begin{array}{l}\text { Gewinnmotivation } \\
\text { Landwirtschaft }\end{array}$ & $\begin{array}{l}\text { Artgerechte Tierhaltung mit } \\
\text { ausreichenden Rechtsvorschriften }\end{array}$ \\
\hline
\end{tabular}

Tierwohlbewusstsein

Glaubwürdigkeit heimlicher Aufnahmen

Vertrauen in staatliche Kontrollmechanismen

\begin{tabular}{|l}
\hline Alter \\
Bildung \\
(Dummy, hohes Bildungsniveau =1) \\
\hline $\begin{array}{l}\text { Geschlecht } \\
\text { (Dummy, weiblich }=1)\end{array}$ \\
$\begin{array}{l}\text { Wohnort } \\
\text { (Dummy, Stadt=1) }\end{array}$ \\
$\begin{array}{l}\text { Ernährungsweise } \\
\text { (Dummy, Fleischkonsum=1) }\end{array}$ \\
\hline $\begin{array}{l}\text { Haustierbesitzer } \\
\text { (Dummy, Haustierbesitzer=1) }\end{array}$ \\
\hline $\begin{array}{l}\text { Mitgliedschaftling zu heimlichen } \\
\text { (Dummy, Mitgliedschaft }=1 \text { ) }\end{array}$
\end{tabular}

Gesellschaftliche Einstellung

zu einer strafrechtlichen

Verschärfung heimlicher

Aufnahmen

Quelle: Eigene Darstellung 


\section{Methodik}

Im April 2017 wurde für die Untersuchung der gesellschaftlichen Wahrnehmung zu heimlichen Aufnahmen eine Onlinebefragung durchgeführt. Dabei wurde darauf geachtet, dass die Verteilung des Alters, der Bildung und des Geschlechts annähernd mit der Verteilung der gesamten deutschen Bevölkerung übereinstimmt. Insgesamt haben 323 Personen mit einer Nettobeteiligung von 37,9\%, an der Befragung teilgenommen. In die statistische Auswertung gingen 292 Probanden ein. Die Differenz ergibt sich aufgrund von auffällig schnellem oder inkonsistentem Antwortverhalten ausgeschlossener Probanden (Döring und Bortz, 2016).

Die gesellschaftliche Einstellung zu heimlichen Aufnahmen wurde mithilfe von mehreren Statements auf Basis der bisher veröffentlichten wissenschaftlichen Erkenntnisse sowie der in den Medien zu findenden typischen Argumentationsmustern operationalisiert. Berücksichtigt wurden kognitive Einstellungskomponenten, die eine Abfragung des subjektiven Wissens ermöglichen (Güttler, 1996). Aufgrund des explorativen Charakters der Untersuchung und denen aus diesem Grund bisher fehlenden Erkenntnissen zur gesellschaftlichen Einstellung heimlicher Aufnahmen wurde in einem ersten Schritt zur Erprobung der Skala eine explorative Hauptkomponentenanalyse mittels Varimax-Rotation durchgeführt. Die sich aus der gesellschaftlichen Einstellung $\mathrm{zu}$ heimlichen Aufnahmen ableitbare gesellschaftliche Einstellung zu einer härteren strafrechtlichen Ahndung wurde direkt mithilfe des Statements „Heimliche Aufnahmen sollten härter bestraft werden“ abgefragt und beinhaltet damit ebenfalls die kognitive Einstellungskomponente.

Mögliche, bereits in Abb. 1 eingeführte Einflussfaktoren auf die gesellschaftliche Einstellung $\mathrm{zu}$ heimlichen Aufnahmen wurden wie folgt in das Modell aufgenommen: Die Konstrukte „Gewinnmotivation der Landwirtschaft“, „,artgerechte Tierhaltung mit ausreichenden Rechtsvorschriften“, „Tierwohlbewusstsein“ und „Vertrauen in staatliche Kontrollmechanismen“ gehen als Multi-Item- Konstrukte in das Modell ein und werden im Rahmen der Gütebeurteilung des Messmodells auf Reliabilität und Validität überprüft. Das Konstrukt „Glaubwürdigkeit heimlicher Aufnahmen“ geht als Single-Item- Konstrukt in das Modell ein. Anhang 1 ist eine Auflistung der Konstrukte mit den dazugehörigen Statements zu entnehmen.

Da es sich bei dieser Studie um eine explorative Arbeit handelt, wurde bei der Fragebogengestaltung explizit darauf geachtet, ausbalancierte Pro- und Contra-Argumente heimlicher Aufnahmen zu berücksichtigen. Die anschließende Datenauswertung erfolgte mit IBM SPSS 24 und SmartPLS 3. Um zu untersuchen, welche Einflussgrößen die 
gesellschaftliche Einstellung zu heimlichen Aufnahmen bestimmen, wurde ein Partial-LeastSquares-Strukturgleichungs-modell berechnet. Dieses multivariate, varianzbasierte Analyseverfahren eignet sich für die Auswertung kausaler Zusammenhänge besonders im Rahmen explorativer Studien. Das Verfahren erzielt auch bei kleineren Stichproben eine hohe Teststärke (HAIR et al., 2017) und fordert keine Annahmen bezüglich der Verteilung der Daten (CASSEL et al., 1999).

\section{Ergebnisse}

\section{Stichprobenbeschreibung}

Die zugrundeliegende Stichprobe ist annähernd repräsentativ für die deutsche Bevölkerung in den Eigenschaften Alter, Bildung und Geschlecht (Tabelle 1). Es wohnen 15,5\% der Befragten in einer Landgemeinde mit weniger als 5.000 Einwohnern, 25,2 \% leben in einer Kleinstadt, 28,6 \% leben in einer Mittelstadt und 30,7 \% der Befragten leben in einer Großstadt. Außerdem essen 94,5\% der Befragten Fleisch, 4,4 \% ernähren sich vegetarisch und 1,0\% verzichtet komplett auf tierische Produkte.

Tabelle 1. Soziodemografische Stichprobenbeschreibung

\begin{tabular}{lcc}
\hline & $\begin{array}{c}\text { Stichprobe } \\
(\mathrm{N}=292)\end{array}$ & $\begin{array}{c}\text { Statistisches } \\
\text { Bundesamt }\end{array}$ \\
\hline Alter & $12 \%$ & $9 \%$ \\
18-25 Jahre & $22 \%$ & $22 \%$ \\
26-40 Jahre & $46 \%$ & $44 \%$ \\
41-65 Jahre & $21 \%$ & $25 \%$ \\
66 und älter & & \\
Geschlecht & $50 \%$ & $49 \%$ \\
Männlich & $50 \%$ & $51 \%$ \\
Weiblich & & \\
Bildung & $1 \%$ & $4 \%$ \\
(noch) kein Schulabschluss & $35 \%$ & $35 \%$ \\
Volks-und Hauptschulabschluss & $32 \%$ & $31 \%$ \\
Mittlere Reife/weiterbildende Schule ohne Abitur & $15 \%$ & $14 \%$ \\
Abitur/Hochschulreife/Fachhochschulreife & $17 \%$ & $17 \%$ \\
Abgeschlossenes Studium & & \\
\hline
\end{tabular}

Quelle: Eigene Berechnungen, Statistisches Bundesamt (2016) 


\section{Gesellschaftliche Bewertung heimlicher Aufnahmen}

Die Ergebnisse der explorativen Vorstudie deuten darauf hin, dass sich die Einstellung zu heimlichen Aufnahmen in drei Dimensionen darstellt. Die extrahierten Faktoren gehen im Anschluss daran in das Strukturgleichungsmodell ein und können wie folgt benannt werden: „Notwendigkeit aus Tierschutzgründen“, „mangelnde Legitimität des tierschützerischen Vorgehens“, „psychisches Leid betroffener Landwirte“. In Tabelle 2 sind die deskriptiven Ergebnisse zur Einstellung heimlicher Aufnahmen sowie zur Einstellung einer härteren strafrechtlichen Ahndung dargestellt. Es zeigt sich, dass mehrheitlich die Auffassung besteht, dass heimliche Aufnahmen aus Tierschutzgründen notwendig sind. Die Legitimität des tierschützerischen Vorgehens beim Filmen von Haltungsbedingungen wird kaum in Frage gestellt. Unsicher sind sich die Befragten allerdings darüber, welche Folgen heimliche Aufnahmen für Landwirte haben können. Der Aussage, dass Landwirte und ihre Familien leiden, wenn Bilder von ihrem Stall in der Öffentlichkeit gezeigt werden, stimmen etwas mehr als ein Drittel der Befragten zu und knapp ein Viertel sind der Meinung, dass Landwirte unter der Angst vor heimlichen Aufnahmen leiden. Eine Notwendigkeit einer härteren strafrechtlichen Ahndung wird kaum gesehen.

Tabelle 2. Deskriptive Statistiken der endogenen Variablen

\begin{tabular}{|c|c|c|c|c|c|c|c|}
\hline \multirow[b]{2}{*}{ Variablen } & \multicolumn{5}{|c|}{ Skala } & \multirow[b]{2}{*}{$\mu^{1,2}$} & \multirow[b]{2}{*}{$\sigma^{3}$} \\
\hline & $\begin{array}{l}\text { Stimme } \\
\text { überhaupt } \\
\text { nicht zu }\end{array}$ & $\begin{array}{l}\text { Stimme } \\
\text { nicht } \\
\mathrm{zu}\end{array}$ & $\begin{array}{l}\text { Teils / } \\
\text { teils }\end{array}$ & $\begin{array}{l}\text { Stimme } \\
\text { zu }\end{array}$ & $\begin{array}{l}\text { Stimme } \\
\text { voll und } \\
\text { ganz zu }\end{array}$ & & \\
\hline \multicolumn{8}{|l|}{ Notwendigkeit aus Tierschutzgründen } \\
\hline $\begin{array}{l}\text { Wenn Tierschützer heimliche Aufnahmen } \\
\text { in landwirtschaftlichen Ställen machen, } \\
\text { helfen sie die Tierhaltung zu verbessern. }\end{array}$ & $1,4 \%$ & $6,5 \%$ & $28,9 \%$ & $34,7 \%$ & $28,5 \%$ & 3,82 & 0,97 \\
\hline $\begin{array}{l}\text { Wenn Tierschützer heimlich Aufnahmen } \\
\text { in landwirtschaftlichen Ställen machen, } \\
\text { denken sie nur an das Wohl der Tiere. }\end{array}$ & $1,0 \%$ & $7,3 \%$ & $26,0 \%$ & $34,3 \%$ & $31,5 \%$ & 3,88 & 0,97 \\
\hline $\begin{array}{l}\text { Wenn Tierschützer heimlich Aufnahmen } \\
\text { in landwirtschaftlichen Ställen machen, } \\
\text { wollen sie die Gesellschaft über } \\
\text { Tierschutzprobleme informieren. }\end{array}$ & $0,7 \%$ & $1,7 \%$ & $15,1 \%$ & $37,3 \%$ & $45,2 \%$ & 4,25 & 0,82 \\
\hline $\begin{array}{l}\text { Sonst können Tierschutzprobleme nicht } \\
\text { aufgedeckt werden. }\end{array}$ & $0,7 \%$ & $7,3 \%$ & $19,4 \%$ & $35,3 \%$ & $37,4 \%$ & 4,01 & 0,96 \\
\hline $\begin{array}{l}\text { Heimliche Aufnahmen sind nötig, weil } \\
\text { der Staat zu wenig kontrolliert. }\end{array}$ & $1,7 \%$ & $6,5 \%$ & $24,7 \%$ & $34,9 \%$ & $32,2 \%$ & 3,89 & 0,99 \\
\hline Gut, dass sich Menschen so für den & $0,7 \%$ & $2,4 \%$ & $20,3 \%$ & $33,0 \%$ & $43,6 \%$ & 4,16 & 0,88 \\
\hline
\end{tabular}

Tierschutz engagieren. 


\begin{tabular}{lcccccccc}
\hline & \multicolumn{6}{c}{ Skala } & & \\
\cline { 2 - 6 } & $\begin{array}{c}\text { Stimme } \\
\text { uberhaupt } \\
\text { nicht zu }\end{array}$ & $\begin{array}{c}\text { Stimme } \\
\text { nicht }\end{array}$ & $\begin{array}{c}\text { Teils / } \\
\text { teils }\end{array}$ & $\begin{array}{c}\text { Stimme } \\
\text { zu }\end{array}$ & $\begin{array}{c}\text { Stimme } \\
\text { voll und } \\
\text { ganz zu }\end{array}$ & $\mu^{1,2}$ & $\sigma^{3}$ \\
\hline $\begin{array}{l}\text { Heimliche Aufnahmen sind wichtig für } \\
\text { den Tierschutz. }\end{array}$ & $0,3 \%$ & $4,5 \%$ & $17,2 \%$ & $36,8 \%$ & $41,2 \%$ & 4,14 & 0,88
\end{tabular}

\section{Mangelnde Legitimität des tierschützerischen Vorgehens}

$\begin{array}{lccccccc}\begin{array}{l}\text { Wenn Tierschützer heimliche Aufnahmen } \\ \text { in landwirtschaftlichen Ställen machen, } \\ \text { haben sie nur ihren eigenen Vorteil im } \\ \text { Sinn. }\end{array} & 21,9 \% & 37,7 \% & 26,0 \% & 11,0 \% & 3,4 \% & 2,36 & 1,05 \\ \begin{array}{l}\text { Wenn Tierschützer heimliche Aufnahmen } \\ \text { in landwirtschaftlichen Ställen machen, } \\ \text { wollen sie nur Spenden bekommen. }\end{array} & 19,0 \% & 42,1 \% & 29,0 \% & 8,3 \% & 1,7 \% & 2,32 & 0,93 \\ \begin{array}{l}\text { Heimliche Aufnahmen verschlechtern das } \\ \text { Image der Landwirtschaft unnötig. }\end{array} & 16,2 \% & 33,7 \% & 37,1 \% & 8,9 \% & 4,1 \% & 2,51 & 1,00 \\ \begin{array}{l}\text { Heimliche Aufnahmen sind unfair } \\ \text { gegenüber den Landwirten. }\end{array} & 19,0 \% & 27,6 \% & 39,7 \% & 11,0 \% & 2,8 \% & 2,51 & 1,00 \\ \begin{array}{l}\text { Heimliche Aufnahmen sind häufig } \\ \text { gefälscht. }\end{array} & 10,0 \% & 33,7 \% & 44,7 \% & 7,9 \% & 3,8 \% & 2,62 & 0,90 \\ \begin{array}{l}\text { Dadurch werden Tiere gefährdet. } \\ \text { Psychisches Leid betroffener Landwirte }\end{array} & 21,0 \% & 42,8 \% & 26,2 \% & 6,6 \% & 3,4 \% & 2,29 & 0,98 \\ \begin{array}{l}\text { Landwirte und ihre Familien leiden, } \\ \text { wenn Bilder von ihrem Stall in der }\end{array} & 8,9 \% & 15,1 \% & 38,4 \% & 30,1 \% & 7,5 \% & 3,12 & 1,04 \\ \begin{array}{l}\text { Offentlichkeit gezeigt werden. } \\ \text { Landwirte leiden unter der Angst vor }\end{array} & 8,6 \% & 24,4 \% & 43,0 \% & 15,8 \% & 8,2 \% & 2,91 & 1,03\end{array}$
heimlichen Aufnahmen.

\section{Notwendigkeit strafrechtlicher Verschärfung}

$\begin{aligned} & \text { Heimliche Aufnahmen sollten härter } \\ & \text { bestraft werden. }\end{aligned}$

\footnotetext{
${ }^{1}$ Auf einer Skala von $1=$ Stimme überhaupt nicht zu bis $5=$ Stimme voll und ganz zu, ${ }^{2}=$ Mittelwert, ${ }^{3}=$ Standardabweichung

Quelle: Eigene Berechnungen
}

\section{Gütebeurteilung des Messmodells}

Das Messmodell zeichnet sich durch die Beziehungen zwischen den Indikatoren und Konstrukten aus und muss auf Reliabilität und Validität überprüft werden (HAIR et al., 2017). In Tabelle 3 sind die Gütekriterien zur Prüfung der Internen-Konsistenz-Reliabilität und Konvergenzvalidität der Multi-Item-Konstrukte zusammengefasst.

Die Reliabilität wird auf Basis der Internen- Konsistenz-Reliabilität mithilfe Cronbachs alpha und Composite Reliabilität beurteilt. Aufgrund der Werte der Composite-Reliabilität, die zwischen 0,70 und maximal 0,95 liegen, kann ausreichende Reliabilität angenommen werden 
(HAIR et al., 2017). Die Validität wird mithilfe der Konvergenzvalidität und der Diskriminanzvalidität beurteilt. Die Konvergenzvalidität betrachtet die Indikatorreliabilität und die durchschnittlich erfasste Varianz. Bei Überprüfung der Indikatorreliabilität mussten einige Indikatoren aufgrund von zu geringen Ladungen entfernt werden (HAIR et al., 2017). In das endgültige Modell gehen nur Indikatoren ein, die eine Ladung von mindestens 0,70 aufweisen. Die in Tabelle 2 dargestellten Statements weisen bereits ausreichende Indikatorreliabilität auf und gehen in das finale Modell ein. Die zugehörigen Faktorladungen sind Anhang $1 \mathrm{zu}$ entnehmen. Die durchschnittlich erfasste Varianz liegt für alle Multi-Item-Konstrukte über dem Grenzwert von 0,50. Zusätzlich wird mithilfe des Fornell-Larcker-Kriteriums und des Heterotrait-Monotrait-Verhältnisses die Diskriminanzvalidität geprüft (HAIR et al., 2017). Die Ergebnisse bestätigen, dass Diskriminanzvalidität angenommen werden kann. Die Reliabilität und Validität des Modells ist auf Basis der untersuchten Gütekriterien gegeben.

\section{Beurteilung des Strukturmodells}

Für die Beurteilung der Ergebnisse des Strukturmodells (Abb. 2) wird die Prognosefähigkeit und die Beziehung zwischen den Konstrukten des Modells geprüft (HAIR et al., 2017). Für die Prognosefähigkeit wird das Bestimmtheitsmaß $\left(\mathrm{R}^{2}\right)$ betrachtet. Die Prüfung der Pfadkoeffizienten erlaubt eine Evaluation der Beziehungen zwischen den Konstrukten des Modells (HAIR et al., 2017). Die Analyse zeigt, dass die „Notwendigkeit aus Tierschutzgründen“ zu 41 \% durch das Modell erklärt werden kann. Das endogene Konstrukt „mangelnde Legitimität des tierschützerischen Vorgehens“ kann zu $26 \%$ durch die in das Modell einfließende Konstrukte erklärt werden. Die „Notwendigkeit einer strafrechtlichen Verschärfung“ kann zu 38 \% durch das Modell erklärt werden. Diese Ergebnisse sind in Anbetracht des explorativen Charakters dieser Studie zufriedenstellend. Das Konstrukt „psychisches Leid betroffener Landwirte“ weist ein Bestimmtheitsmaß von $10 \%$ auf. Dieses Ergebnis verdeutlicht, dass dieses Konstrukt vermehrt von anderen nicht in dieser Studie erhobenen Indikatoren beeinflusst wird.

Die Betrachtung der signifikanten Pfadkoeffizienten zeigt, dass die Einstellung gegenüber heimlichen Aufnahmen durch das „Tierwohlbewusstsein“, der „Gewinnmotivation der Landwirtschaft“, der ,,artgerechten Tierhaltung mit ausreichenden Rechtsvorschriften“ und der wahrgenommene „Glaubwürdigkeit heimlicher Aufnahmen“ beeinflusst wird (Abb. 2). Alle in Abschnitt 2.3 dargestellten Hypothesen wurden getestet. In Abbildung 2 sind allerdings nur die statistisch signifikanten Einflussfaktoren der Beziehungen dargestellt. 
Das Konstrukt „Notwendigkeit aus Tierschutzgründen“ lässt sich mit abnehmender Relevanz durch die Konstrukte „Gewinnmotivation der Landwirtschaft“, „Tierwohlbewusstsein“, „Glaubwürdigkeit heimlicher Aufnahmen“, und ,artgerechte Tierhaltung mit ausreichenden Rechtsvorschriften“ erklären. Wird der Landwirtschaft vermehrt eine rein ökonomische Motivation nachgesagt, dann werden heimliche Aufnahmen aus Tierschutzgründen als nötig wahrgenommen. Steigt das „Tierwohlbewusstsein“ an, werden heimliche Aufnahmen auch vermehrt als notwendig aus Tierschutzgründen wahrgenommen. Einen Einfluss hat außerdem die Glaubwürdigkeit heimlicher Aufnahmen. Nimmt sie zu, so werden auch heimliche Aufnahmen als notwendiger angesehen. Wird hingegen die Tierhaltung als artgerecht angesehen, dann werden heimliche Aufnahmen weniger als nötig empfunden.

Das Konstrukt „mangelnde Legitimität des tierschützerischen Vorgehens“ wird mit abnehmender Effektstärke durch die Konstrukte ,artgerechte Tierhaltung mit ausreichenden Rechtsvorschriften“, „Tierwohlbewusstsein“ und „Glaubwürdigkeit heimlicher Aufnahmen“ erklärt. Wird die landwirtschaftliche Tierhaltung als artgerecht betrachtet und werden gesetzliche Vorschriften als ausreichend angesehen, wird die Legitimität des tierschützerischen Vorgehens vermehrt angezweifelt. Nimmt das Bewusstsein für das Wohlergehen landwirtschaftlicher Nutztiere $\mathrm{zu}$ und werden heimliche Aufnahmen als glaubwürdig wahrgenommen, nehmen hingegen Zweifel am legitimen Vorgehen von Tierschützern ab.

Das Konstrukt „psychisches Leid betroffener Landwirte“ wird durch das Konstrukt „Tierwohlbewusstsein“ erklärt. Ein zunehmendes Bewusstsein für das Wohlergehen von landwirtschaftlichen Nutztieren geht einher mit abnehmendem Verständnis für das Leid betroffener Landwirte.

Die „Notwendigkeit einer strafrechtlichen Verschärfung“ wird signifikant von der „mangelnden Legitimität des tierschützerischen Vorgehens“ beeinflusst. Wird die Legitimität der Tierschützer, die heimliche Aufnahmen produzieren, in Frage gestellt, wird eine Verschärfung der strafrechtlichen Maßnahmen als notwendiger angesehen.

Persönliche Merkmale (Alter, Bildung, Geschlecht, Wohnort, Ernährungsweise, Haustierbesitz, Mitgliedschaft Tierschutz/Tierrechtsorganisation) sowie das „Vertrauen in staatliche Kontrollmechanismen“ haben keinen signifikanten Einfluss auf die Einstellung zu heimlichen Aufnahmen und deren strafrechtlichen Verschärfung. 
Tabelle 3: Gütekriterien zur Prüfung der Internen-Konsistenz-Reliabilität und Konvergenzvalidität

\begin{tabular}{lccc}
\hline Konstrukt & $\begin{array}{c}\text { Cronbach's } \\
\text { alpha }\end{array}$ & $\begin{array}{c}\text { Composite } \\
\text { Reliabilität }\end{array}$ & $\begin{array}{c}\text { Durchschnittlich } \\
\text { erfasste Varianz }\end{array}$ \\
\hline Gewinnmotivation der Landwirtschaft & 0,527 & 0,808 & 0,678 \\
Vertrauen in staatliche Kontrollmechanismen & 0,796 & 0,895 & 0,789 \\
Tierwohlbewusstsein & 0,549 & 0,815 & 0,688 \\
Artgerechte Tierhaltung mit ausreichenden & 0,785 & 0,875 & 0,699 \\
Rechtsvorschriften & & & \\
Notwendigkeit aus Tierschutzgründen & 0,900 & 0,921 & 0,607 \\
Mangelnde Legitimität des tierschützerischen Vorgehens & 0,864 & 0,898 & 0,595 \\
Psychisches Leid betroffener Landwirte & 0,613 & 0,837 & 0,720 \\
\hline
\end{tabular}

Quelle: Eigene Berechnungen

Abbildung 2: Signifikante Konstruktbeziehungen

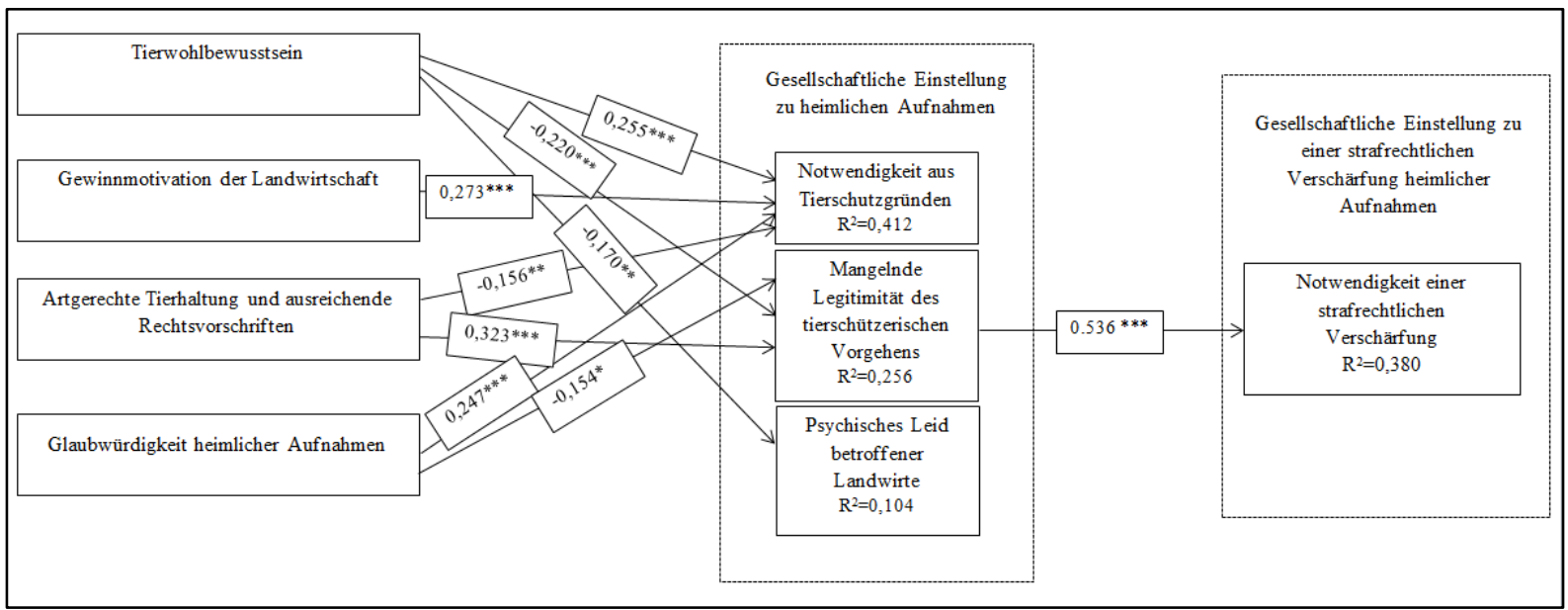

Dargestellt sind nur Konstruktbeziehungen mit mind. $\mathrm{P} \leq 0.05, *=$ signifikant $\leq 0,05 ; * *=$ hoch signifikant $\leq$ 0,$01 ; * * *=$ höchst signifikant $\leq 0,001$

Quelle: Eigene Berechnungen und Darstellung

\section{Diskussion und Fazit}

Die vorliegende Untersuchung verdeutlicht, dass sich die gesellschaftliche Einstellung zu heimlichen Aufnahmen in drei Faktoren unterteilen lässt. Zum einen konnte das Konstrukt „Notwendigkeit aus Tierschutzgründen“ identifiziert werden. Die zugrundeliegenden Indikatoren weisen neben fehlenden und nicht ausreichenden Kontrollmechanismen vor allem auf die Wichtigkeit heimlicher Aufnahmen zum Schutz landwirtschaftlicher Nutztiere hin. Dieses Konstrukt erfährt hohe gesellschaftliche Zustimmung. Bereits ZANDER et al. (2013) stellen eine gesellschaftliche Forderung nach einer Überarbeitung und Intensivierung von Kontrollmechanismen in der Tierhaltung fest. Es zeigt sich, dass heimliche Aufnahmen als 
Hilfsmittel angesehen werden, um wahrgenommene Lücken im staatlichen Kontrollsystem zu schließen und das Wohlergehen landwirtschaftlicher Nutztiere zu verbessern.

Zum anderen konnte das Konstrukt „,mangelnde Legitimität des tierschützerischen Vorgehens“ extrahiert werden. Gesellschaftliche Zweifel an der Legitimität der Arbeit von Tierschützern im Kontext heimlicher Aufnahmen sind kaum vorhanden. Dieses Ergebnis bestätigt das hohe gesellschaftliche Vertrauen in die Arbeit von NGOs (GfK, 2017).

Außerdem konnte das Konstrukt „psychisches Leid betroffener Landwirte“ identifiziert werden. Gesellschaftlich ist die Zustimmung zu diesem Faktor sehr ausgeglichen. Viele Befragte haben keine eindeutige Position zur emotionalen Belastung der Landwirte durch heimliche Aufnahmen. Viele sind sich unsicher, ob Landwirte unter heimlichen Aufnahmen leiden oder nicht. Die gesellschaftliche Unsicherheit liegt möglicherweise darin begründet, dass der Beruf des Landwirts zwar grundsätzlich als positiv wahrgenommen wird, das gesamte Produktionssystem der Landwirtschaft aber gesellschaftliche Kritik erfährt (KANTAR EMNID, 2017). Die Forderung nach heimlichen Aufnahmen aufgrund der Unzufriedenheit mit landwirtschaftlichen Produktionspraktiken steht der positiven Einstellung zu Landwirten gegenüber. Dieser gesellschaftliche Zwiespalt führt möglicherweise zu einem ausgeglichenen Antwortverhalten.

Das Konstrukt „Notwendigkeit einer strafrechtlichen Verschärfung“ zeigt, dass nur ein geringer Teil der Befragten eine härtere strafrechtliche Ahndung fordert. Dieses Ergebnis findet sich auch in Studien aus den USA wieder, die herausstellen, dass Gesetze, die heimliche Aufnahmen bestrafen, in der amerikanischen Gesellschaft kaum Zustimmung finden (ASPCA, 2012). Im Gegensatz zu den USA, wo einige Bundesstaaten gleichwohl solche Gesetze eingeführt haben (SHEA, 2014), stimmen in Deutschland rechtliche Bewertungen und gesellschaftliche Einstellungen überein. Die festgestellte positive gesellschaftliche Einstellung zu heimlichen Aufnahmen aus Tierschutzgründen spiegelt sich in den vorliegenden strafrechtlichen Entscheidungen wider (OBERLANDESGericht NAUMBURG, Urt. v. 22.02.2018, Az. 2 Rv 157/17), in denen es zu Freisprüchen kam. Gleichwohl wird eine härtere strafrechtliche Ahndung bereits auf politischer Ebene diskutiert (KOALITIONSVERTRAG, 2018).

Anhand der Ergebnisse wird deutlich, dass die Einstellung zu heimlichen Aufnahmen vor allem von dem gesellschaftlichen Gedanken des Schutzes und der Förderung des Wohlergehens landwirtschaftlicher Nutztiere beeinflusst wird. Das zunehmende gesellschaftliche Bewusstsein für das Wohlergehen landwirtschaftlicher Nutztiere (ZANDER et al., 2013; SPILLER et al., 2016) führt dazu, dass heimliche Aufnahmen aus Tierschutzgründen als notwendig erachtet werden und die legitime Vorgehensweise von Tierschützern kaum angezweifelt wird. Auswirkungen, 
die heimliche Aufnahmen auf Landwirte haben können, werden hingegen weniger beachtet, wenn das Bewusstsein für das Wohlergehen von Nutztieren zunimmt.

Die zunehmende gesellschaftliche Kritik an der modernen Nutztierhaltung (z.B. ZANDER et al., 2013; WBA, 2015) ist u.a. in der gesellschaftlichen Wahrnehmung einer rein ökonomisch orientierten Landwirtschaft begründet. Die gesellschaftliche Einschätzung einer fehlenden emotionalen Beziehungen zwischen Landwirt und Nutztier sowie der von der Gesellschaft wahrgenommene rein gewinnorientierte Fokus von Landwirten werden als nicht vertretbar angesehen (ZANDER et al., 2013). Mit dieser Wahrnehmung geht ebenfalls die zunehmende Überzeugung, heimliche Aufnahmen seien aus Tierschutzgründen nötig, einher. Wird die landwirtschaftliche Tierhaltung hingegen als artgerecht angesehen, werden heimliche Aufnahmen weniger als notwendig angesehen und die Legitimität der Arbeit von Tierschützern vermehrt angezweifelt. Da allerdings eine zunehmende gesellschaftliche Kritik an der Nutztierhaltung zu beobachten ist (z.B. ZANDER et al., 2013; WBA, 2015), ist die Betrachtung des umgekehrten Wirkungszusammenhangs geeigneter, d.h. die zunehmende gesellschaftliche Kritik an der modernen Nutztierhaltung geht einher mit einer Zustimmung zu heimlichen Aufnahmen. Insgesamt zeigt sich eine Kombination aus gesellschaftlicher Kritik an der landwirtschaftlichen Produktion in Kombination mit entsprechender Zustimmung zu Undercoveraufnahmen.

Die oftmals vonseiten der Landwirtschaft angezweifelte Glaubwürdigkeit verdeckter Aufnahmen (BBV, 2016) beeinflusst die Einstellung zu heimlichen Aufnahmen ebenfalls. Heimliche Aufnahmen werden als notwendiger erachtet, wenn derartige Aufnahmen auch als glaubwürdig eingestuft werden. Wird hingegen die Glaubwürdigkeit von Undercoveraufnahmen angezweifelt, wird auch die Legitimität des Vorgehens von Tierschützern angezweifelt.

Persönliche Merkmale beeinflussen die Einstellung zu heimlichen Aufnahmen entgegen den aufgestellten Hypothesen (MCKENDREE et al., 2014; RoBBINS et al., 2016; Su und MARTENS, 2017) nicht. Obwohl Tierschutz- und Tierrechtsorganisationen zunehmend als wichtige Informationsquelle der Gesellschaft in Tierschutzfragen gelten (MCKENDEE et al., 2014), konnte in dieser Studie kein Einfluss von Mitgliedschaften in Tierschutzorganisationen auf die Einstellung zu heimlichen Aufnahmen ermittelt werden.

Die gesellschaftlich kaum geforderte Verschärfung der strafrechtlichen Normen für heimliche Aufnahmen wird direkt nur von der wahrgenommenen Legitimität des Vorgehens von Tierschützern beeinflusst. Das zunehmende gesellschaftliche Vertrauen in die Arbeit von 
Tierschützern (GfK, 2017) geht einher mit einer Ablehnung einer strafrechtlichen Ahndung heimlicher Aufnahmen.

Die Ergebnisse dieser Untersuchung bestätigen damit die bisherigen wissenschaftlichen Erkenntnisse, die einerseits auf eine gesellschaftliche Befürwortung heimlicher Aufnahmen hinweisen (TIPLADY et al., 2013, 2015; LIEBE et al., 2017 und LIEBE und Jahnke, 2017) und andererseits auf eine gesellschaftliche Ablehnung einer härteren strafrechtlichen Ahndung hindeuten (ASPCA, 2012; RoBBINS et al., 2016). Es kann gezeigt werden, dass es der Gesellschaft vor allem um den Schutz und das Wohlergehen der Tiere geht. Bei der Einstellungsbildung zu Undercoveraufnahmen spielt die Einstellung zur tierhaltenden landwirtschaftlichen Produktion die wichtigste Rolle. Eine negative Einstellung gegenüber der landwirtschaftlichen Produktion und den bestehenden Rechtsvorschriften bzgl. der Tierhaltung geht i.d.R. mit einer Zustimmung zu heimlichen Aufnahmen einher.

Anhand der gesellschaftlichen Zustimmung zu heimlichen Aufnahmen wird die Diskrepanz zwischen Landwirtschaft und Gesellschaft deutlich. Dabei kann der Zuspruch, den heimliche Aufnahmen erfahren, als Indikator für die gesellschaftliche Betrachtung der modernen landwirtschaftlichen Produktion angesehen werden. Die im Rahmen dieser Untersuchung identifizierten Ursachen der gesellschaftlichen Einstellung zu heimlichen Aufnahmen können helfen, Diskrepanzen zwischen Landwirtschaft und Gesellschaft durch gezielte Maßnahmen zu verringern. Möglicherweise eignet sich der Ausbau von Kontrollmechanismen dazu, die konfliktäre Situation zu entschärfen. Verstärkte Kontrollmechanismen könnten bereits vor dem Eindringen von Tierschützern Missstände entdecken und Maßnahmen zum Schutz der Tiere ergreifen. Diese Maßnahmen würden der gesellschaftlichen Forderung nach einer Intensivierung staatlicher Kontrollmechanismen nachkommen (ZANDER et al., 2013) und möglicherweise die gesellschaftliche Kritik reduzieren. Der Zuspruch zu heimlichen Aufnahmen verdeutlicht, dass die Gesellschaft an Einblicken in die landwirtschaftliche Produktion interessiert zu sein scheint und eine transparentere Produktion fordert (KANTAR EMNID, 2017). Da heimliche Aufnahmen die landwirtschaftliche Realität nur bedingt abbilden und manipulierbar sein können, scheint es angezeigt, andere Maßnahmen anzubieten, um dem Transparenzinteresse der Gesellschaft an der Landwirtschaft gerecht zu werden. Dabei ist zu überlegen, ob Landwirte ihre Ställe der Öffentlichkeit zugänglicher machen und vermehrt in das Gespräch mit interessierten Personengruppen einsteigen sollten (WBA, 2015).

Diese Arbeit liefert erste Erkenntnisse darüber, wie heimliche Aufnahmen gesellschaftlich bewertet und wodurch sie beeinflusst werden. Der explorative Aufbau dieser Untersuchung auf Basis einer relativ begrenzten Stichprobengröße ermöglicht erste wissenschaftliche 
Erkenntnisse zur gesellschaftlichen Einstellung heimlicher Aufnahmen. Es bedarf weiterer Untersuchungen, die sich mit den unterschiedlichen Sichtweisen der Beteiligten (Tierschutzorganisationen, Staat, Landwirte) befassen. Heimliche Aufnahmen haben viele Ausprägungen (z. B. (keine) Aufdeckung von Tierschutzvergehen), können auf unterschiedlichste Weise entstehen (z. B. Whistleblowing, Einbruch mit/ohne Personen/Sachschaden) und können unterschiedliche Inhalte haben (z. B. direkter Umgang der Mitarbeiter mit Tieren, Haltungsbedingungen, Medikamenteneinsatz). Da heimliche Aufnahmen in dieser Untersuchung nicht differenziert betrachtet werden, ist nicht auszuschließen, dass die unterschiedlichen Ausprägungen auch gesellschaftlich anders bewertet werden. Dies ist weiteren Untersuchungen vorbehalten.

Danksagung: Diese Studie ist im Rahmen des Promotionsprogramms „Animal Welfare in Intensive Livestock Production Systems“ entstanden. Wir danken dem Niedersächsischen Ministerium für Wissenschaft und Kultur für die finanzielle Unterstützung 


\section{Literatur}

ANIMAL ViSUALS (2015): Ag-Gag Laws and Factory Farm Investigations Mapped. In: http://www.animalvisuals.org/projects/data/investigations, Abruf: 27.09.2017.

ASPCA (American Society for the Prevention of Cruelty in Animals) (2012): Research shows americans overhelming support investigations to expose animal abuse on industrial farms. In: http://www.aspca.org/sites/default/files/public_memo_aspca_farm_animal_ research_ag_gag.pdf, Abruf: 27.09.2017.

AwATER-ESPER, S. (2017): Politiker üben Kritik an Stalleinbrüchen. In: top agrar online, https://www.topagrar. com/news/Home-top-News-Politiker-ueben-Kritik-anStalleinbruechen-8290347.html, Abruf: 01.10.2017.

BBV (Bayerischer Bauernverband) (2016): Pressemitteilung - Stalleinbruch: Aufruhr im Putenstall. In: https://www.bayerischerbauernverband.de/pm60-stalleinbruch, Abruf: 21.09.2017.

CASsel, C., P. HACKL and A.H. Westlund (1999): Robustness of partial leasts quares method for estimating latent variable quality structures. In: Journal of Applied Statistics 26 (4): 435-466.

DETER, A. (2017): Stalleinbrüche treffen die ganze Familie. In: top agrar online, https://www.topagrar.com/news/ Home-top-News-Stalleinbrueche-treffen-die-ganzeFamilie-8309446.html, Abruf: 21.09.2017.

DETER, A. (2016): Stall Eindringlinge freigesprochen. In: top agrar online, https://www.topagrar.com/news/Home-top-News -Stall-Eindringlinge-freigesprochen4718410.html, Abruf: 21.09.2017.

DETER, A. (2013): Tierschützer greifen Schweinehalter an. In: top agrar online, https://www.topagrar.com/news/Home-top- News-Straathof-Kirketerp-und-vanGennip-sollen-gegen -Tierschutzverordnung-verstossen-1296890.html?page=1, Abruf: 25.09.2017.

DÖRING, N. und J. BORTZ (2016): Forschungsmethoden und Evaluation in den Sozial- und Humanwissenschaften. 5. Auflage. Springer Verlag, Berlin, Heidelberg: 589-590.

FRONTAL 21 (28.03.2018): In: https://www.zdf.de/politik/ frontal-21/elterntierhaltung-beimasthuehnern-100.html.

FRONTAL 21(13.02.2018): In: https://www.zdf.de/politik/frontal-21/ frontal-21-vom-13februar-2018-100.html.

GfK (Gesellschaft für Konsum-, Markt- und Absatzforschung e.V.) (2017): Global Trust Report - Vertrauen in Institutionen und Branchen. Druckhaus Haspel, Erlangen. 
GÜTTLER, P.O. (1996): Sozialpsychologie: Soziale Einstellungen, Vorurteile, Einstellungsänderungen. Oldenbourg Wissenschaftsverlag, München.

Hair, J., J.F. Hair, Hult, G.T.M., Ringle, C.M., SARStedt, M., Richter, N.F., HAufF, S. (2017): Partial Least Squares Strukturgleichungsmodellierung - Eine anwendungsorientierte Einführung. Verlag Franz Vahlen GmbH, München.

HIRT, A., C. MAISACK und J. Moritz (2016): Tierschutzgesetz: TierSchG. 3. Auflage. Vahlen Verlag, München.

JoHnSON, J. (2014): On the Ethics of Ag-Gag Legislation and Undercover Investigations. In: Northern Plains Ethics Journal 2 (1): 30-39.

KANTAR EMNID (2017): Das Image der deutschen Landwirtschaft, Ergebnisbericht März 2017. In: https://media.repro-mayr.de/79/668279.pdf, Abruf: 14.08.2018.

KOALITIONSVERTRAG DEUTSCHLAND (2018): In: https://www.cdu.de/system/tdf/media/dokumente/koalitionsvertrag_2018.pdf?file=1.

KOEHLER, L. (2013): Survey shows transparency is important to consumers. In: farmWeekNow.com, http://farmweeknow.com/story-survey-shows-transparen cyimportant-consumers-0-101157, Abruf: 22.09.2017.

KRÜSKEN, B. (2017): Stalleinbrüche rechtlich ächten. In: Grüner Dienst, Deutscher Bauernverband, Ausgabe vom 17.08.2017, KW 33.

LiEBE, U., B. JAhNKE und U. HeITHOLT (2017): Tier- Mensch-Beziehungen, Einblicke in die Bevölkerungsmeinung. In: LOEWE Forschungsschwerpunkt Tier- MenschGesellschaft (Hrsg.): Vielfältig verflochten: Interdisziplinäre Beiträge zur TierMensch-Relationalität. Auflage 1. transcript Verlag, Bielefeld.

LIEBE, U. und B. JAHNKE (2017): Legaler und illegaler Tierrechtsaktivismus. In: LOEWE Forschungsschwerpunkt Tier-Mensch-Gesellschaft (Hrsg.): Vielfältig verflochten Interdisziplinäre Beiträge zur Tier-Mensch- Relationalität. Auflage 1. transcript Verlag, Bielefeld.

MARCEAU, J.F. (2015): Ag gag past, present, future. In: Seattle University Law Review 38 (4): $1317-1344$.

MCKendREe, M.G.S., C.C. CRONEY and N.J.O. WidMAR (2014): Effects of demographic factors and the information sources on United States consumer perceptions of animal welfare. In: Journal of Animal Science 92 (7): 3161-3173.

Robbins, J.A., B. Franks, D.M., WeAry and M.A.G. vOn KeyserlingK (2016): Awareness of ag-gag laws erodes trust in farmers and increases support for animal welfare regulations. In: Food Policy 61 (Mai): 121-125. 
Roscher, M. (2012): Tierschutz- und Tierrechtsbewegung - ein historischer Abriss. In: Aus Politik und Zeitgeschichte 8-9 (2012): 34-40.

SCHLECHT, S., F. AlbersmeIER und A. SPILlER (2010): Eine Analyse medialer Frames bei Konflikten im ländlichen Raum - das Beispiel landwirtschaftlicher Bauvorhaben. In: Kayser, M., J. Böhm und A. Spiller (Hrsg.): Die Ernährungswirtschaft in der Öffentlichkeit - Social Media als neue Herausforderung der PR. Cuvillier, Göttingen: 335-364.

SHEA, M. (2014): Punishing Animal Rights Activists for Animal Abuse: Rapid Reporting and the new wave of ag-gag laws. In: Columbia Journal of Law and Social Problems 48 (3): 337-371.

SonNTAG, W. und A. SPILlER (2016): Prozessqualitäten in der WTO: Ein Vorschlag für die reliable Messung von moralischen Bedenken. Diskussionspapier des Departments für Agrarökonomie und Rurale Entwicklung der Georg-August-Universität Göttingen, No. 1603.

SpILler, A., M. vON MEYER-HÖFER und W. SOnNTAG (2016): Gibt es eine Zukunft für die moderne konventionelle Nutztierhaltung in Nordwesteuropa? Diskussionspapier des Departments für Agrarökonomie und Rurale Entwicklung der Georg-AugustUniversität Göttingen, No. 1608.

STATISTISCHES BundESAMT (2016): Statistisches Jahrbuch. Wiesbaden. In: https://www.destatis .de/DE/Publikationen/StatistischesJahrbuch/StatistischesJahrbuch2016.pdf.

StEPHAN, R. (2017): Terror oder Tierschutz: Was rechtfertigt Stalleinbrüche? In: Land \& Forst online, https://www.agrarheute.com/landundforst/betrieb-familie/betriebsfue hrung/terror-tierschutz-rechtfertigt-stallein brueche-534851, Abruf: 25.09.2017.

SU, B. and P. MARTENS (2017): Public attitudes toward animals and the influential factors in contemporary China. In: Animal Welfare 26 (2): 239-247.

TIPLADY, C.M., D.B. WALSH and C.J.C. PHILlIPS (2015): Ethical Issues concerning the public viewing of Media Broadcasts of Animal Cruelty. In: Journal of Agricultural and Environmental Ethics 28 (4): 635- 645.

TiPlady, C.M., D.B. WALsh and C.J.C. PhILIPS (2013): Public Response to Media Coverage of Animal Cruelty. In: Journal of Agricultural and Environmental Ethics 26 (4): 869885. 
TONSOR, G.T. and N.J. OLYNK (2010): U.S. Meat Demand: The Influence of Animal Welfare Media Coverage. Agricultural Experiment Station and Cooperative Extension Service, Kansas State University, Manhatten.

WBA (Wissenschaftlicher Beirat für Agrarpolitik beim Bundesministerium für Ernährung und Landwirtschaft) (2015): Wege zu einer gesellschaftlich akzeptierten Nutztierhaltung. Gutachten. Berlin.

Zander, K., F. Isermeyer, D. BÜGelt, I. Christoph-Schulz, P. SAlamon und D. Weible (2013): Erwartungen der Gesellschaft an die Landwirtschaft. Gutachten beauftragt durch Stiftung Westfälische Landschaft, Braunschweig. 


\section{Anhang}

Anhang 1. Konstrukte und dazugehörige Statements

\begin{tabular}{lll}
\hline Konstrukt und Statements & Mittelwert & $\begin{array}{l}\text { Standard- } \\
\text { abweichung }\end{array}$ \\
\hline
\end{tabular}

\section{Gewinnmotivation der Landwirtschaft ${ }^{1}$}

Bei der Tierhaltung geht es nur um den Gewinn des

$3,75 \quad 0,935 \quad 0,792$

Landwirtes.

Es gibt viele schwarze Schafe unter den Tierhaltern.

$4,15 \quad 0,909 \quad 0,854$

\section{Artgerechte Tierhaltung mit ausreichenden Rechtsvorschriften ${ }^{1}$}

Den Tieren in der Landwirtschaft geht es heute besser als $\quad 2,82$ früher.

$2,82 \quad 1,056 \quad 0,813$

Die Tiere in der Landwirtschaft werden artgerecht gehalten.

$2,72 \quad 0,893$

In Deutschland sind Tiere durch gesetzliche Vorschriften

2,67

1,043

0,847 ausreichend geschützt.

\section{Tierwohlbewusstsein ${ }^{1}$}

Mir ist es egal, wie Tiere in der Landwirtschaft gehalten werden. (umcodiert)

$4,38 \quad 0,862 \quad 0,852$

Das Wohlergehen von Nutztieren in der Landwirtschaft ist mir wichtig.

$4,23 \quad 0,794 \quad 0,807$

Glaubwürdigkeit heimlicher Aufnahmen2

Wie glaubwürdig finden Sie heimlich gemacht Aufnahmen in landwirtschaft-lichen Ställen?

\section{Vertrauen in staatliche Kontrollmechanismen}

Wie wirkungsvoll sind Ihrer Meinung nach staatliche

\section{Notwendigkeit aus Tierschutzgründen ${ }^{1}$}

Wenn Tierschützer heimliche Aufnahmen in

landwirtschaftlichen Ställen machen, helfen sie, die

Tierhaltung zu verbessern.

Wenn Tierschützer heimlich Aufnahmen in

landwirtschaftlichen Ställen machen, denken sie nur an das Wohl der Tiere.

Wenn Tierschützer heimlich Aufnahmen in landwirtschaftlichen Ställen machen, wollen sie die

Sonst können Tierschutzprobleme nicht aufgedeckt werden.

Heimliche Aufnahmen sind nötig, weil der Staat zu wenig

Gut, dass sich Menschen so für den Tierschutz engagieren.

$\begin{array}{lll}4,16 & 0,879 & 0,879 \\ 4,14 & 0,881 & 0,854\end{array}$

Heimliche Aufnahmen sind wichtig für den Tierschutz. 


\begin{tabular}{lll}
\hline Konstrukt und Statements & Mittelwert & $\begin{array}{l}\text { Standard- } \\
\text { abweichung }\end{array}$ \\
\hline
\end{tabular}

\section{Mangelnde Legitimität des tierschützerischen Vorgehens ${ }^{1}$}

Wenn Tierschützer heimliche Aufnahmen in
landwirtschaftlichen Ställen machen, haben sie nur ihren

$2,36 \quad 1,048 \quad 0,798$
eigenen Vorteil im Sinn.

Wenn Tierschützer heimliche Aufnahmen in

$2,32 \quad 0,932 \quad 0,743$

landwirtschaftlichen Ställen machen, wollen sie nur Spenden bekommen.

Heimliche Aufnahmen verschlechtern das Image der

Landwirtschaft unnötig.

Heimliche Aufnahmen sind unfair gegenüber den Landwirten.

Heimliche Aufnahmen sind häufig gefälscht.

$2,03 \quad 0,982 \quad 0,764$

Dadurch werden Tiere gefährdet.

$\begin{array}{lll}2,51 & 1,009 & 0,782 \\ 2,62 & 0,907 & 0,769 \\ 2,29 & 0,983 & 0,774\end{array}$

\section{Psychisches Leid betroffener Landwirte ${ }^{1}$}

Landwirte und ihre Familien leiden, wenn Bilder von ihrem Stall in der Öffentlichkeit gezeigt werden.

Landwirte leiden unter der Angst vor heimlichen Aufnahmen.

$\begin{array}{lll}3,12 & 1,048 & 0,871 \\ 2,91 & 1,035 & 0,826\end{array}$

\section{Notwendigkeit strafrechtlicher Verschärfung ${ }^{1}$}

Heimliche Aufnahmen sollten härter bestraft werden.

$2,38 \quad 1,164 \quad 1,000$

\footnotetext{
${ }^{1}$ auf einer Skala von „1 = stimme überhaupt nicht zu“ bis , $5=$ stimme voll und ganz zu“; ${ }^{2}$ auf einer Skala von „, 1 $=$ gar nicht glaubwürdig“ bis, $5=$ voll und ganz glaubwürdig“; ${ }^{3}$ auf einer Skala von, $1=$ gar nicht wirkungsvoll"“ bis, $5=$ voll und ganz wirkungsvoll“; ${ }^{4}$ auf einer Skala von „, $1=$ vertraue gar nicht“ bis, $5=$ vertraue voll und ganz"

Quelle: eigene Berechnungen
} 


\title{
I.2 Public Perceptions of Undercover Investigations in Livestock Farming: An End that Justifies the Means?
}

\author{
Autoren: Maureen Schulze, Antje Risius, Achim Spiller \\ Der Beitrag ist erschienen in Animal Welfare 2021, 30(1), 39-47. \\ https://doi.org/10.7120/09627286.30.1.039
}

\begin{abstract}
Photos taken secretly of livestock production systems, representing animal welfare violations, regularly appear in the media and initiate discussions about whether it is legitimate to override legal regulations in order to document animal welfare standards. This paper focuses on the public perspective and compares different forms of undercover investigation, weighing animal welfare against the invasion of farmers' privacy. For this purpose, an exploratory online survey was conducted in Germany $(n=292)$. Participants were carefully selected to ensure that age range, education level and sex reflected the distribution of the society as a whole. In a splitsample survey, each participant was confronted with three scenarios. The scenarios were mapped using pictures showing various levels of animal abuse combined with small information segments describing the degree of invasion of farmers' privacy. Participants evaluated the scenarios for their perceived legitimacy and whether entering the premises should be punished. All forms of undercover investigation were perceived as legitimate by most respondents. Perceived legitimacy was considerably higher when animal abuse was uncovered. Except where damage to property was involved, which was mostly considered as unacceptable, harsher punishment for animal welfare organisations generally obtained little social approval. The public's increasing awareness of farm animal welfare overruled social norms regarding farmers' privacy, and thereby demonstrated the importance of animal welfare in society. Approval of undercover investigations indicated that changes in housing and handling conditions as well as improvement in control mechanisms are necessary to increase animal welfare and thereby public acceptance of livestock production.
\end{abstract}

Acknowledgements: The authors are grateful to the Ministry of Science and Arts in Lower Saxony, Germany for funding the PhD Programme 'Animal Welfare in Intensive Livestock Production Systems', which led to this work being completed. 


\title{
Teil II - Präferenzen und Zahlungsbereitschaften für nachhaltig erzeugte Fleischwaren
}

\section{II.1 Do consumers prefer pasture-raised dual-purpose cattle when considering meat products? A hypothetical discrete choice experiment for the case of minced beef}

\author{
Autoren: Maureen Schulze, Achim Spiller, Antje Risius \\ Dieser Beitrag ist erschienen in Meat Science 2021, 177, 108494. \\ https://doi.org/10.1016/j.meatsci.2021.108494
}

\begin{abstract}
Livestock production is criticised for animal welfare conditions and its impact on the environment. Pasture-raised dual-purpose cattle may be able to provide an opportunity for more sustainable livestock production. Despite societal interest and substantial grazing opportunities in several regions of northern Europe, the market share of sustainably produced beef is currently low. This study investigated consumer preferences and willingness-to-pay for pasture-raised beef from dual-purpose cattle. Data were obtained from a hypothetical choice experiment $(\mathrm{n}=$ 513), attributing the type of husbandry (stable-based, pasture-raised, pasture-raised using nature conservation areas), breed (no description, single-purpose, dual-purpose), production method (conventional, organic), origin (locally produced, produced in Germany), and price (5.98, $11.98,17.98,23.98 € / \mathrm{kg}$ ), and were analysed using random parameter logit modelling. The most important overall attribute was 'type of husbandry' followed by 'breed', indicating consumers' concerns for animal welfare and naturalness. Our analyses revealed a clear preference for pasture-raised dual-purpose cattle, demonstrating great market potential for animal welfare-friendly meat products.
\end{abstract}

Acknowledgements: The project on which this contribution is based was conducted with research-oriented learning in collaboration with the student Pia Skroch.

Funding: This work was supported by the Federal Office for Agriculture and Food, Germany (BLE 2818301416) and the Ministry of Science and Arts (MWK) in Lower Saxony, Germany. The authors are responsible for the content of this publication. 
Teil III - Der Lebensmitteleinzelhandel als Gestalter nachhaltiger

Konsum- und Produktionsmuster

III.1 Food Retailers as Mediating Gatekeepers between Farmers and Consumers in the Supply Chain of Animal Welfare Meat - Studying Retailers' Motives in Marketing Pasture-Based Beef

Autoren: Maureen Schulze, Achim Spiller, Antje Risius

Dieser Beitrag ist erschienen in Food Ethics 2019, 3(1-2), 41-52.

https://doi.org/10.1007/s41055-019-00040-w 


\section{Abstract}

Although there is increasing public criticism of intensive livestock production, the market share of meat with an animal welfare standard exceeding legal requirements remains small. Food retailers, in their role as gatekeepers, can influence changes in production and consumption patterns. Their strategic role between farmers and consumers allows them to control commodity, information and value flow and therefore places them into a key position when it comes to the distribution of meat with a higher animal welfare standard. The aim of this explorative study is to identify factors which motivate food retailers to take on the marketing of products of increased animal welfare standards, in this case, pasture-based beef. Nine in depth interviews were conducted with representatives of the food retail industry. The interviews took place in June 2018, followed a structured guideline and were transcribed verbatim. The transcripts were categorized and evaluated using qualitative content analysis. Results showed that food retailers are driven by both extrinsic and intrinsic motives. The main extrinsic motive is the perceived customer demand. Consciousness for animal welfare and the regional production cycles, including close connection between farmers and retailers are inherently intrinsic motives. Interestingly, the interviewed retailers show a high personal interest and moral obligation with regard to sourcing and marketing pasture-based beef. As such, this research finds innovative retailers, who take on a new role in sustainable food systems which exceed classical distribution functions and may have a considerable effect in transforming the food system. 
Teil III - Der Lebensmitteleinzelhandel als Gestalter nachhaltiger Konsum- und

Produktionsmuster

\section{Introduction}

There is increasing public interest in ethical foods, such as organic, fair trade or animal products with extraordinary animal welfare standards (Valor et al. 2014). Particularly when it comes to meat, a growing interest in increased standards such as outdoor access, freedom of movement and freedom to express normal behavior can be observed (e.g. Boogaard and Bock 2006; Van Loo Ellen et al. 2014). In order to meet the changing demands of society, a change in husbandry conditions and the implementation of products with an animal welfare standard exceeding legal requirements (in the following we refer to these products as "animal welfare meat") seems necessary. Although various consumer studies already predict an enormous market potential, the actual market share of such products in Germany remains small (Moewius et al. 2018; Pirsich and Theuvsen 2017).

When trying to close the gap between the public's interest and the actual demand, food retailers, as the main meat-source for consumers in Germany (Kohlmüller and Koch 2018), might serve as a helpful actor to change production and consumption patterns. In their influential gatekeeper position, retailers have the ability to significantly affect consumers' purchasing behavior by deciding what products are offered and which information about those products is provided (Esbjerg et al. 2016). In this special role, the food retail trade is also considered the "ecological gatekeeper", who is paying attention to the marketing of sustainable products (Hansen 1993). Additionally, in contrast to farmers, retailers are able to react to changes in consumer demand quickly and with a high degree of flexibility (Franz and Spiller 2010).

As innovations are usually associated with a particular degree of uncertainty (Hall 2002), it can be assumed that a retailer's decision to sell animal welfare meat is risky as well. The product needs additional financial and mental input in order to communicate its process attributes to the customers (Fernkvist and Eskelund 2014). As it is not possible for consumers to personally experience animal welfare as a product attribute, neither before nor after consumption, retailers have to provide additional trustworthy information in order to increase consumers' willingness to pay for such products (Risius and Hamm 2016). The fact that, to date, there is no general definition of what animal welfare exactly means, increases the difficulty of marketing products with an increased animal welfare standard. Also, more effort in communicating the added value of these products to consumers is needed. Even though consumer interest in high-quality meat products has increased in recent years, communicating small prices for meat products is a common concept in the marketing thereof (Jones et al. 2007; Franz and Spiller 2010). For this reason, retailers who try to foster an implementation of meat products originating from 
Teil III - Der Lebensmitteleinzelhandel als Gestalter nachhaltiger Konsum- und

production with higher animal welfare standards must use alternatives to the usual strategy of cost leadership. Once a retailer has developed a communicational concept, he is confronted with the risk of competitors copying it (Hall 2002) and thereby reducing his competitive advantage. Additionally, the decision to sell animal welfare meat should be evaluated on a longterm perspective, as it is connected to changes along the whole supply chain (Franz and Spiller 2010), especially long-term adaption of new requirements for farmers.

In the past, the food retail trade has already initiated considerable efforts to change the food system with regard to higher animal welfare standards. A well-known example of food retailers using their power is the discontinuation of eggs originating from caged hens. After a big German grocery concern set the ball rolling in 2004, many other retailers also adopted the discontinuation, leading to an EU-wide ban on caging laying hens. This decision affected farmers and consumers alike: the former had to change their production methods, while the purchase decisions of the latter were directly influenced as well. A less radical action is the labelling of meat products recently introduced by large German food retailers, which are intended to make customers aware of production conditions (Herrmann 2019). These examples show that the food retail trade is able to implement private standards for the production of animal-based food which go beyond the legal framework, in order to address societal animal welfare concerns. However, the reasons behind business decisions to embark on such projects are not finally understood.

In literature, the voluntary commitment of a company is referred to as Corporate Social Responsibility (CSR), a term which, at its core, can be described as a company's initiative to include social needs in its business operations (e.g. Bartels and Winter 2015; Piacentini et al. 2000). Since introducing meat with a higher animal welfare standard requires voluntary additional business commitment, we can adopt the theoretical background of existing CSR motives to get a first idea of retailer's motivation behind the marketing of such products. Applying the scientific results derived from research on CSR, we can assume that food retailers are driven by both extrinsic (e.g. financial) and intrinsic (e.g. ethical) motives (e.g. Graafland and van de Ven 2006).

Based on Milton Friedman's well-known quote that "there is one and only one social responsibility of business - to use its resources and engage in activities designed to increase its profits" (Friedman 1970), the companies' efforts on CSR can be regarded as extrinsically motivated. Many scientific studies have already considered CSR efforts as "business case" (e.g. Caroll and Shabana 2010) and examined the connection between voluntary commitment and 
Teil III - Der Lebensmitteleinzelhandel als Gestalter nachhaltiger Konsum- und

financial success (e.g. Margolis and Walsh 2003), but conclusions differ. Regarding firms proenvironmental behavior short-term extrinsic motives describe direct effects on their profitability because of cost reduction in the production process (e.g. Aguilera et al. 2007; Graafland and Mazereeuw-Van der Duijn Schouten 2012).

One of the main long-term extrinsic reasons for voluntary engagement in food retailing is reputation. In regard to this, therefore, food quality, food safety, labour standards but also environmental and animal welfare standards are important to retailers' initiatives (Fulponi 2006). In contrast to that the basis of the intrinsic motive is the assumption that a business "has an ethical duty to give back to society" (Brønn and Vidaver-Cohen 2008). When looking at intrinsic motivation, the role of the food retail manager himself would appear to be particularly important. This is especially the case in the independent retail trade, where the responsibility associated with the ability to act increases the incentive to voluntarily take the initiative (Williams and Schaefer 2013). Research into the intrinsic motivation of managers implementing pro-environmental strategies shows, that basic personal values play an important role in the decision-making process. Values regarding managers self-transcendence might have a positive impact on their voluntary engagement, whereas self-enhancing values might do the opposite (Williams and Schaefer 2013). Additionally, several studies (e.g. Papagiannakis and Lioukas 2012; Cordano and Frieze 2000; Stern 2000) already identified a connection between managers pro environmental attitude and its influence on their company's environmental behavior. Some analyses even show, that an entrepreneur's wish to "do the right thing" influences his implementation of CSR measures more than the possibility of financial gain (Graafland and van de Ven 2006). Even though it is a known fact that voluntary business commitment is motivated by both extrinsic and intrinsic factors, it is not finally understood what motivates those in the food retail trade to include meat with additional animal welfare standards. Since the production of food relies on natural resources, the food sector plays a special role (Genier et al. 2009). Next to the production of high-quality and safe food, it also has to consider environmental impacts (Maloni and Brown 2006). Further, meat production involves the housing and handling of livestock, which raises further ethical questions and additionally highlight food retailers' crucial role with regard to the marketing of animal welfare meat.

Considering the retailers' power to change both consumption and production patterns, it seems essential to further understand retailers' decision-making process. As retailers seem to be risk averse and they rather invest in a new market segment if a well working example already exists, it seems essential to further understand their drivers and moral obligations. Based on this, it can 
Teil III - Der Lebensmitteleinzelhandel als Gestalter nachhaltiger Konsum- und

be assumed that especially intrinsic motives help to overcome initial uncertainty and risk (Franz and Spiller 2010). If this is the case, incentives that promote the marketing of animal welfare meat should be reconsidered (Graafland and van de Ven 2006).

The aim of this analysis is to close the research gap described above and identify motives that foster retailers' decision to start investing in the marketing of meat with an animal welfare standard that goes beyond legal regulations.

\section{Methods}

A case study on the marketing of pasture-based beef by retailers is used in this study. This method seems useful, as retailers' motives to sell pasture-based beef products has not been analyzed before (Pullman and Dillard 2010). We have used a qualitative research approach, including nine in-depth expert interviews, a method which is often chosen for investigating perceptions and experiences of individuals (Rombach and Bitsch 2015). Seven retailers and two experts from the wholesale market, who all are part of a German cooperatively organized supermarket group, were interviewed.

The retailers were selected, because they were already selling pasture-based beef at the time of selection. This was important in order to investigate what motivates retailers to start the marketing of these products. Additionally, an important aspect was the communication with people who have decision-making power over the meat assortment, as previous studies point out the importance of their experiences and motivations (e.g. Williams and Schaefer 2013). Additionally, we selected retailers based in the south of Germany, because the case product to date is only produced and marketed there. The respective managers all have access to obtaining their products through the cooperative and benefit from the cooperative's support and marketing experience; however, they are not necessarily obliged to do so. It is the managers' task to make final decisions about selling a product and how to manage their marketing concept.

In order to investigate underlying motives of retail decisions, it was important to include two experts from the wholesale market in our study and profit from their overview and insight into the market situation. They were also selected because of their experience in the supervision of grocery stores that already sell pasture-based beef in the south of Germany and because they are also part of the cooperative.

The chosen product, pasture-based beef, is suitable as an exemplary product to investigate retailers' motives. Because of its production process, it is able to satisfy the changing demands of society regarding meat products. The animals are on pasture from May to October, and living 
Teil III - Der Lebensmitteleinzelhandel als Gestalter nachhaltiger Konsum- und

as part of a herd gives them the freedom to express normal behavior and the freedom of movement. To date, the term "pasture-based beef" is not legally protected in Germany. The definition originates from the producer organization and the associated retail trade company. Additionally, the production process of this product is in line with European guidelines for organic livestock production.

The phone-interviews took place in June 2018. They followed a semi-structured guideline and were audio-recorded. On average, the interviews lasted around $20 \mathrm{~min}$. However, the variations within the duration of the interviews were considerable. Two interviews (one retailer and one supervisor) lasted between 10 and 15min, three interviews (two retailers and one supervisor) lasted around $30 \mathrm{~min}$. The remaining interviews lasted around $20 \mathrm{~min}$. Even though the interviews were conducted on a date previously set by the respondents, the variation in duration was due to the respondents' lack of time. Presumably in face-to-face interviews, instead of telephone interviews, the retailers would have prioritized the interviews and planned more time. Subsequently, the interviews were transcribed and analyzed with a qualitative content analysis according to Mayring (2010). In order to identify underlying motives, we first used an inductive process, known as open coding, and added new categories if differences to prior data were found. In a second step, we tried to examine whether the motives are of extrinsic or intrinsic origin. The core questions of the semi-structured interviews were the following:

Why did you decide to market pasture-based beef?

How do you try to make customers aware of the product?

What is the most important issue when you think about the marketing of pasture-based beef? In addition, more general questions about how they perceive their meat product range, how they perceive customer interest in such products, as well as what problems they encounter and how they cope with them were added.

\section{Results}

The results show, that extrinsic as well as intrinsic motives might influence retailers' decision to sell pasture-based beef. The interviews show that retailers' main extrinsic motive is the perceived customer demand. Consciousness for animal welfare and regional production, connected with a close personal connection to farmers are identified as intrinsic motives. Additionally, the interviews show retailers' high personal interest regards sourcing and marketing pasture-based beef. In the following results are presented in detail. 
Teil III - Der Lebensmitteleinzelhandel als Gestalter nachhaltiger Konsum- und

External motives focusing on increasing profits on a short-term perspective were not mentioned by retailers. However, a few of the interviewed retailers stated the importance of sales figures and the success of their product range. Two retailers even pointed out the sales problems involved in the marketing of pasture-based beef.

"Selling pasture-based beef is difficult. If we have a terrific offer on rump steak, for example, they [the customers] will of course buy other products. " $(B, 55)$.

In contrast to that, when analyzing the interviews with the wholesale experts, it was found that they keep in mind the short-term financial success through marketing of pasture-based beef and see a clear need for optimization.

\section{"And they [the sales figures] are successful. But there is still room for improvement." (L,13).}

Long-term economic motives were often mentioned in the interviews with both retailers and wholesale experts. The aim to satisfy consumer demand is paramount, and the majority of interviewees had included pasture-based beef in their product range because customers had explicitly asked for it.

During the interviews, also other external factors which might influence their decision to market pasture-based beef and can hardly be influenced were named. Both, seasonal influences and site-specific influences (e.g. urban versus rural location) are playing an important role. According to most respondents' assessments, high-priced products are more likely to be marketed in high-income, urban areas. Only one retailer suggested that customers in rural areas are aware and appreciative of farmers' efforts and are, therefore, more willing to pay for such products. One retailer also stated that younger people, especially young families, appreciate pasture-based beef. In addition, neighboring discounters or direct marketers further influence the marketing success of meat products they forces a retailer to offer cheaper meat in order to remain competitive. Neighboring direct marketers also increase the difficulties for retailers to market the premium product successfully, as customers then prefer to buy the product directly from the producer. Demand can also be driven by recently uncovered scandals, while other given influences are seasonal. It is, for instance, much easier to sell barbecue meat in summer while meat better suited for roasting is easier sold winter.

Internal motives for including pasture-based beef into the product range are the retailers' interest in the welfare of farm animals. Their interest in this regard reflects a combination of 
Teil III - Der Lebensmitteleinzelhandel als Gestalter nachhaltiger Konsum- und

the perceived interest in responsible livestock production of their customers but also their personal intrinsic interest in the welfare of the animals. Especially important for most retailers is how the cattle are fed. However, topics such as housing conditions and slaughtering are important aspects as well when it comes to the animal welfare-oriented meat production.

"We have always thought it was good to have the animals out on grass, and only fed on grass outside etc. That has always been part of our business philosophy, and that's why we have included it in our product range. And it has been a success.,$(E, 25)$.

The increased accessibility of the farmer is also an important aspect for the interviewed retailers. In case of pasture-based beef production, the retailers are strongly in favor of the perceived accessibility of the farmer, which is guaranteed by regional production in the south of Germany. However, we do not know how often retailers actually have contact with farmers or if they only feel connected to them because of the regional production.

"And when I see the farmers struggling up the hillsides and how hard they work every day, I am right behind them." (B, 25).

Interviews with wholesale experts did, however, not identify these internal motives. Additionally, the interviews showed the retailers' general interest in premium meat products. The majority of the interviewed retailers stressed the importance of also including other premium meat products into their range, in addition to pasture-based beef. They focus not only on the conditions in which livestock is reared, but also on other process attributes such as a more elaborate maturation. They stated that pasture-based beef has particular sensory and optical properties which result from the alternative production conditions.

Furthermore, the interviews showed the retailers' interest in new products and new marketing possibilities. They realized the importance of regularly including new products in their range and acting as drivers for the implementation of new marketing possibilities.

"We've often launched new products on the market and pushed them and it's easy. You sit down together and think about the best way to do it (..) and then off you go.” (E, 41). “And you must never stand still, you have to go forward. That's another thing we've learnt (..), that you can never sit back and relax.” (K, 21).

Additionally, retailers were found to be very innovative in terms of their communication with customers. Providing additional information regarding the production conditions is necessary 
Teil III - Der Lebensmitteleinzelhandel als Gestalter nachhaltiger Konsum- und

to draw attention to the special product attributes. In this regard, the high degree of animal welfare, and the accessibility of the farmer are particularly highlighted. Therefore, they use creative ideas to inform their customers about the special features of the product. Providing text and picture material through brochures as well as short films at the sale point in the store were mentioned often in this respect. Also, offering tasting events and organizing excursions where customers can visit farms are tools used by retailers to draw the customers' attention and provide information. One retailer has created his own logo to promote the product, while another is thinking about introducing innovative product variations.

"Or you can offer new innovative products using pasture-based beef. I could imagine a burger patty, for example." $(K, 25)$.

When selling pasture-based beef, particular problems arise as a result of the unequal marketing possibilities for the different parts of the animal. The interviewed retailers also react in an innovative way to such unavoidable problems. Some make use of other processing methods (e.g. boiling down less popular parts) in order to guarantee that the animal is marketed as a whole. As a further point, it became clear that the interviewed retailers highly value the work of their employees and are aware that the successful marketing of pasture-based beef largely depends on the employees' interest and motivation to sell the product. In their view, the personal interaction and information exchange acts as a promising tool which completes their concept. Therefore, they reliably inform their staff about production methods and the added values of the product. Some of them actually introduced their staff directly to the farmers.

“The success of a program for pasture-based beef depends on your employees (..).

[They have] to learn about the philosophy and get to know the farm too, practically feel the passion for the product. And then, they have to be able to pass on their enthusiasm to others." $(K, 21)$.

Additionally, most interviewed retailers highly value the farmers' work and try to take on a supporting rather than a dictating role when looking for problems in the marketing of pasturebased beef.

"We have to carry on giving them our support. So that they [the farmers] continue to get good money from us and from the consumer." (B, 41). 
Teil III - Der Lebensmitteleinzelhandel als Gestalter nachhaltiger Konsum- und

While one of the experts was also in favor of the close cooperation with farmers, the other expert found a cooperation between farmers to be more useful.

"I think we must support the small farmers. How can they produce better meat?"(A, 49).

Lastly, in contrast to retailers', the wholesale experts addressed the benefits for the countryside and the environment as a result of marketing pasture-based beef.

In summary, both extrinsic and intrinsic motives were identified. The interviews hardly differ between retailers and wholesale experts. However only for retailers it was possible to identify clear intrinsic motives. In addition to motives, willingness to implement communication tools and other personal interests could be identified, which presumably facilitate the inclusion of pasture-based beef.

\section{Discussion}

As extrinsic and intrinsic motives often influence one other (Graafland and van de Ven 2006) the precise identification of extrinsic and intrinsic origin of the identified motives was difficult. However, as results of CSR research would suggest, both intrinsic and extrinsic motives that might foster the decision whether to sell pasture-based beef or not are identified. Parallels can be observed between the interviewed retailer's extrinsic motivation and findings of previous studies investigating CSR motives. However, the results regarding their intrinsic motivation offer insights that might help to further reconsider incentive structures in order to support the marketing of meat with an additional animal welfare standard. In the following first extrinsic, intrinsic motives and finally retailers' personal interest will be discussed in regard to how and why the retailers invest in the marketing of pasture-based beef.

With regard to the retailers' perspective, short-term economic aims seem to play only a minor role. As selling pasture-based beef is not directly connected to cost reduction, which is one of the main short-term motives as it directly influences firms profitability (e.g. Aguilera et al. 2007; Graafland and Mazereeuw-Van der Duijn Schouten 2012), this results is not surprising. It can be assumed that interviewed retailers are aware of the fact that the marketing of pasturebased beef is a long-term investment affecting the whole supply chain. Additionally, they are aware that a higher reputation that might go along with market pasture-based beef can only in the long-run lead to improved financial success (Miles and Covin 2000). 
Teil III - Der Lebensmitteleinzelhandel als Gestalter nachhaltiger Konsum- und

Identified intrinsic motives are more directly linked to the products process attributes. This study reveals an interest in increased animal welfare standards and its regional origin including a perceived close connection to the farmers as inherently intrinsic motive. Based on the wellknown connection of attitude and behavior (Honkanen and Olsen 2006), it can be assumed that the retailers' awareness for animal welfare issues and their appreciation of regional product origin might be the most influential product related attributes that affect retailers' decisionmaking process regarding the marketing of pasture-based beef. Since the marketing of such products is coupled with high risk and uncertainty, the identified intrinsic motivation might help pioneers to overcome these problems. The interviewed retailers might act as 'marketing role models', motivating followers to invest. As investment barriers regarding the marketing of new products will decrease with rising market shares (Franz and Spiller 2010), the importance of motivated pioneers should not be underestimated. Previous economic literature shows that extrinsic motives negatively impact the occurrence of intrinsic motives, so, a financial support for retailers to promote the marketing of pasture-based beef might support their intrinsic motivation (Graafland and van de Ven 2006). As intrinsic motivation helps to not only invest in less costly or unimportant issues (Lee 2008), this should be noted when reconsidering incentive structures for the widespread marketing of animal welfare meat. Retailers seemed to include their personal moral obligation into their decision-making process. Therefore, retailers did not only keep their financial goal in mind but rather used their position in order to foster sustainable production and consumption patterns. This changed role should be investigated in further studies because it seems to be a promising step towards a more sustainable food system. Additionally, based on studies regarding firm's pro-environmental behavior (e.g. Papagiannakis and Lioukas 2012; Cordano and Frieze 2000; Stern 2000), it can be concluded that personal attitudes can also positively influence the orientation of globally acting companies.

In contrast to the interviewed retailers, the wholesale experts were far more interested in the products ability to satisfy the public's changing demands regarding livestock production. Personal values and attitudes, however, only play a minor role. This might reflect their advisory role, which differs from that of the local managers, who often include their personal values when designing corporate philosophy, as is normal in small companies (Williams and Schaefer 2013). Additionally, interviewees are aware that selling pasture-based beef needs a lot of communication efforts. Presumably, these additional efforts will be implemented more frequently by intrinsically motivated retailers. Our findings show that in-store information, through brochure-texts and videos or staff and event marketing, not only leads consumers to 
Teil III - Der Lebensmitteleinzelhandel als Gestalter nachhaltiger Konsum- und

buy the product, but also enables them to get to know the story surrounding the product. In contrast to traditionally used cost leadership strategies, the concept of the interviewed retailers regarding the marketing of pasture-based beef rather resembles the "interaction approach" often used in organic food stores (Stockebrand et al. 2008). The interviewed retailers are trying to market the product by drawing the consumers' attention to the approachability of the farmer and highlighting the advantages thereof. This parallel might also explain why one interviewed retailer stated, that direct marketers are one main competitor in the marketing of pasture-based beef. Since collaboration along the supply chain seems to be useful in order to keep the costs down (Franz and Spiller 2010), the interviewed retailers' intrinsic interest in farmers work might help to achieve common goals. Within the cooperation, retailers can benefit from farmers' knowledge, set practically orientated quality standards and still grant the product access to the consumer market. However, when evaluating the retailers' interest in marketing innovative high-quality products, it should also be considered that they might also benefit from cooperatives' superordinate CSR efforts, which already focus on similar values and provide them with new ideas. Although the interviewees are able to decide which products they want to sell, the organizational help available and marketing experience might emphasize ethical issues. The reputation the cooperative has already built up might help the interviewed retailers to sell pasture-based beef (McWilliams and Siegel 2001).

Additionally, the interviewed retailers largely focus on their employees. It can, therefore, be assumed that appreciation of their employees also plays a vital role in the decision-making process. Retailers who are not aware of the importance of their employees, might not be interested in selling a communication intensive product such as pasture-based beef, whose success benefit from personal interaction between employee and customer. Also, this finding highlights that although our study focused on the marketing of pasture-based beef, the interviewed retailers also consider aspects such as social and labor standards - aspects of increased importance when regarding retailers' reputation (Fulponi 2006).

However, although food retailing has a big impact on future consumption and production patterns, the responsibility for a change towards a more sustainable food system lies in the hands of all actors along the supply chain (Gjerris et al. 2016). In spite of this, a functioning pasture-based beef supply chain is reliant on the cooperation of all involved participants. Without farmers who back the product, or customers who buy the product, the efforts of the food retail industry remain futile. Results of previous studies show, however, that farmers and consumers are also intrinsically convinced that the implementation of animal welfare in 
Teil III - Der Lebensmitteleinzelhandel als Gestalter nachhaltiger Konsum- und

livestock production is a personal concern (Weber et al. 2008; Hansson and Lagerkvist 2015; Padel and Foster 2005).

When discussing a change in livestock production with regard to increased animal welfare, it must be considered that all forms of food production impact the environment in one way or the other. When discussing the increase of climate and environmental problems, consumption and production of meat, especially beef, cannot be seen as sustainable in general (Tjärnemo and Södahl 2015). This equally applies to the extensive production of meat products, which also does not seem viable at the current rate of human consumption. 
Teil III - Der Lebensmitteleinzelhandel als Gestalter nachhaltiger Konsum- und

\section{Conclusion and Limitations}

The retailers' position between farmers and consumers has been widely researched, and their power to influence the supply chain is undisputed (e.g. Esbjerg et al. 2016). In the recent past, studies have shown that retailers are increasingly setting voluntary private product standards that go beyond the legal requirements (Fulponi 2006). The rather inflexible production conditions in agriculture have to meet changing requirements of the food retail trade. Results of this study reflect retailers' personal interest in animal welfare and their connection to the region. The study provides insights on how to promote animal welfare meat as part of a sustainable agriculture production. Apart from achieving more successful marketing, the motives and efforts of the food retail trade can also help both consumers and farmers to change consumption and production habits.

Retailers that make marketing choices based on financial and intrinsic motives and are additionally supervised by experts who know the consumer demands, are presumably more engaged in the development of innovative initiatives that take even more ethical concerns than animal welfare into account. As such, this research shows that the retailers' role goes beyond the classical distribution function. In contrast to the well-known gatekeeper position, interviewed representatives did not simply decide whether they want to market pasture-based beef.

It is important to realize that it is the structure of the organization which enables the retailers to support and promote such projects. All the interviewed retailers have sole responsibility for their product range. More centralized stores with a different organizational structure may not be able to implement products that are in line with the personal values of their staff. Another limitation is that the study was conducted only in the south of Germany.

If future studies show that the decisions of the food retail trade are indeed driven by intrinsic motives, it is important to rethink incentive structures. A follow-up quantitative survey based on the results of this study, could provide an important contribution to the sustainable change of consumption and production structures by the food retail trade.

Acknowledgements: The authors gratefully acknowledge funding from the "Ministry of Food and Agriculture (BMEL)" and the "Ministry of Science and Arts" in Lower Saxony, Germany. 
Teil III - Der Lebensmitteleinzelhandel als Gestalter nachhaltiger Konsum- und

\section{References}

Aguilera, Ruth V., Deborah E. Rupp, Cynthia A. Williams, and Jyoti Ganapathi. 2007. Putting the S back in corporate social responsibility: a multilevel theory of social change in organizations. Academy of Management Review 32 (3): 836-863.

Bartels, Joe Machiel J. Reinders, and Mariet van Haaster-de Winter. 2015. Perceived sustainability initiatives: retail managers' intrinsic and extrinsic motives. British Food Journal 117 (6): 1720-1736.

Boogaard, Birgit K. Simon J. Oosting, and Bettina B. Bock. 2006. Elements of societal perception of farm animal welfare: a quantitative study in the Netherlands. Livestock Science 104 (1-2): 13-22.

Brønn, Peggy S., and Deborah Vidaver-Cohen. 2008. Corporate motives for social initiative: legitimacy, sustainability, or the bottom line. Journal of Business Ethics 87: 91-109.

Caroll, Archie B., and Kareem M. Shabana. 2010. The business case for corporate social responsibility: a review of concepts, research and practice. International Journal of Management Reviews 12 (1): 58-105.

Cordano, Mark, and Irene H. Frieze. 2000. Pollution reduction preferences of U.S. environmental managers: applying Ajzen's theory of planned behavior. Academy of Management Journal 43 (4): 637-641.

Esbjerg, Lars, Steve Burt, Hannah Pearse, and Viviane Glanz-Chanos. 2016. Retailers and technology-driven innovation in the food sector: caretakers of consumer interests or barriers to innovation? British Food Journal 118 (6): 1370-1383.

Fernkvist, Fredrik, and Lena Eskelund. 2014. Credence and the effect on consumer liking of food - a review. Food Quality and Preference 23 (Part C): 340-356.

Franz, Anabell Marie von Meyer, and Achim Spiller. 2010. Prospects for a european animal welfare label from the German perspective: supply chain barriers. International Journal on Food System Dynamics 4: 318-329.

Friedman, Milton. 1970. The social responsibility of business is to increase its profits. The New York Time Magazine 13 September 1970.

Fulponi, Linda. 2006. Private voluntary standards in the food system: the perspective of major food retailers in OECD countries. Food Policy. 31 (2006): 1-13.

Genier, Claudia Mike Stamp and Mark Pfitzer. 2009. Corporate social responsibility for agroindustries development. In: Agro-industries for development, ed. Carlos A. da Silva, Doyle Baker, Andrew W. Shepherd, Chakib Jenane, and Sergio Miranda-da-Cruz, 223-252. Bodmin: MPG Books Group. 
Teil III - Der Lebensmitteleinzelhandel als Gestalter nachhaltiger Konsum- und

Gjerris, Mickey, Christian Gamborg, and Henrik Saxe. 2016. What to buy? On the complexity of being a critical consumer. Journal of Agricultural and Environmental Ethics 29: 81102.

Graafland, Johan, and Bert van de Ven. 2006. Strategic and moral motivation for corporate social responsibility. The Journal of Corporate Citizenship 22: 111-123.

Graafland, Johan, and Corrie Mazereeuw-Van der Duijn Schouten. 2012. Motives for corporate social responsibility. De Economist 160: 377-369.

Hall, Bronwyn H. 2002. The financing of research and development. Oxford Review of Economic Policy 18 (1): 35-51.

Hansen, Ursula. 1993. Ökologisches Marketing im Handel. In Eberhard Seidel, Heinz Strebel, ed. Betriebliche Umweltökonomie, 448-479. Wiesbaden: Gabler.

Hansson, Helena, and Carl J. Lagerkvist. 2015. Identifying use and non-use values of animal welfare: evidence from Swedish dairy agriculture. Food Policy 50: 36-42.

Herrmann, Wiebke 2019. Einheitliche Haltungskennzeichnung für Fleisch jetzt im Handel. Agrarheute. https://www.agrarheute.com/tier/einheitliche-haltungskennzeichnungfuer-fleisch-handel-552820. Accessed 02 April 2019.

Honkanen, Pirjo Bas Verplanken, and Svein O. Olsen. 2006. Ethical values and motives driving organic food choice. Journal of Consumer Behavior 5 (5): 420-430.

Jones, Peter, Daphne Comfort, and David Hillier. 2007. What's in store? Retail marketing and corporate social responsibility. Marketing Intelligence and Planning 25 (1): 17-30.

Kohlmüller, Matthias, and Tim Koch. 2018. Markt Bilanz - Vieh und Fleisch, ed. In AMI (Argrarmarkt Informationsgesellschaft). Bonn: Medienhaus Plump GmbH.

Lee, Ming - Dong P. 2008. A review of theories of corporate social responsibility: its evolutionary path and the road ahead. International Journal of Management Reviews 10 (1): 53-73.

Maloni, Michael J., and Michael E. Brown. 2006. Corporate social responsibility in the supply chain: an application in the food industry. Journal of Business Ethics 68 (1): 35-52.

Margolis, Joshua D., and James P. Walsh. 2003. Misery loves companies: rethinking social initiatives by business. Administrative Science Quarterly 48 (2): 268-305.

Mayring, Philipp. 2010 Qualitative Inhaltsanalyse - Grundlagen und Techniken. Weinheim and Basel: Beltz.

McWilliams, Abagail, and Donald Siegel. 2001. Corporate social responsibility: A theory of the firm perspective. The Academy of Management Review 26 (1): 117-127. 
Teil III - Der Lebensmitteleinzelhandel als Gestalter nachhaltiger Konsum- und

Miles, Morgan .P., and Jeffrey G. Covin. 2000. Environmental marketing: A source of reputational, competitive, and financial advantage. Journal of Business Ethics 23: 299-311.

Moewius, Joyce Peter Röhring, Diana Schaack, Christine Ramphold, Hans-Josef Brzukalle, Frank Gottwald, Karin Stein-Bachinger, MarkusWolter, and Jürn Sanders. 2018. Zahlen, Daten, Fakten - Bio-Branche 2018, ed. In BÖLW (Bund Ökologischer Lebensmittelwirtschaft e.V.). Berlin: Spree Print.

Padel, Susanne, and Carolyn Foster. 2005. Exploring the gap between attitudes and behavior: understanding why consumers buy or do not buy organic food. British Food Journal 107 (8): 606-625.

Papagiannakis, Giorgos, and Spyros Lioukas. 2012. Values, attitudes and perceptions of managers as predictors of corporate environmental responsiveness. Journal of Environmental Management 100: 41-51.

Piacentini, Maria, Lynn MacFadyen, and Douglas Eadie. 2000. Corporate social responsibility in food retailing. International Journal of Retail and Distribution Management 28 (11): 459-469.

Pirsich, Wiebke Louisa V. Hardenberg, and Ludwig Theuvsen. 2017. Eine empirische Analyse zum Angebot von Tierwohl-Fleisch in Fleischerfachgeschäften. Berichte über Landwirtschaft 95 (2).

Pullman, Madeleine.E., and Jesse Dillard. 2010. Value based supply chain management and emergent organizational structures. International Journal of Operations \& Production Management 30 (7): 744-771.

Risius, Antje, and Ulrich Hamm. 2017. The effect of information on beef husbandry on consumers' preference and willingness to pay. Meat Science 124 (2017): 9-14.

Rombach, Meike, and Vera Bitsch. 2015. Food movements in Germany: slow food, food sharing, and dumpster diving. International Food and Agribusiness Management Review 18 (3): 1-24.

Stern, Paul C. 2000. Toward a coherent theory of environmentally significant behavior. Journal of Social Issues 56 (3): 407-424.

Stockebrand, Nina, Nina S. Berner, and Achim Spiller. 2008. Regionalmarketing im Naturkostfachhandel. Göttingen: Cuvillier.

Tjärnemo, Heléne, and Liv Södahl. 2015. Swedish food retailers promoting climate smarter food choices - trapped between visions and reality? Journal of Retailing and Consume Services 24 (May): 130-139. 
Teil III - Der Lebensmitteleinzelhandel als Gestalter nachhaltiger Konsum- und

Valor, Carmen, Isabel Carrero, and Raquel Redondi. 2014. The influence of knowledge and motivation onsustainable label use. Journal of Agricultural and Environmental Ethics 27 (4): 591-607.

Van Loo Ellen J. Vincenzina Caputo Rodolfo M. Nayga and Wim Verbeke. 2014. Consumer's evaluation of sustainability labels on meat. Food Policy 49(Part 1):137150.

Weber, Klaus, Kathryn L. Heinze, and Michaela DeSoucey. 2008. Forage for thought: Mobilizing codes in the movement for grass-fed meat and dairy products. Administrative Science Quarterly 53 (3): 529-567.

Williams, Sarah, and Anja Schaefer. 2013. Small and medium sized enterprises and sustainability: managers' values and engagement with environmental and climate change issues. Business Strategy and the Environment 22 (3): 173-186 
Teil III - Der Lebensmitteleinzelhandel als Gestalter nachhaltiger Konsum- und Produktionsmuster

III.2 Challenges and Motives in Food Retail Cooperatives' Approach to Supporting Alternative Niche Paths Using the Case of Locally Produced Grass-Fed Beef

Autoren: Maureen Schulze, Antje Risius, Achim Spiller 


\begin{abstract}
Situated between consumers and farmers, food retailers are key actors in transitioning towards more sustainable meat production and consumption. Modern food retail is characterised by a high degree of standardisation and mass production. In the meat sector in particular, there is high price pressure and low-quality differentiation, resulting in a limited supply of sustainably produced meat. However, recently, retail cooperatives have started initiatives aiming to introduce the collaboration with local farmers and thus, the marketing of local food. In doing so, they deviate from the existing meat marketing approach and might foster a transition towards more sustainable meat production and consumption. The aim of this study was to investigate initial challenges and motives for food retail cooperatives to invest in a new approach to marketing sustainably produced meat. Conceptually, our contribution is based on the multi-level perspective on social-technical transitions. In a case study, this research focused on the leading German food retail cooperative's efforts to sell a locally produced meat product, including several sustainable product attributes (i.e. locally produced grass-fed beef). A mixedmethods approach was used, comprising 12 qualitative interviews with food retailer representatives and a quantitative survey taken by 55 retailers. The results showed that the marketing of locally grass-fed beef is hampered by supply chain-related challenges and marketing-related challenges, but that food retailers' intrinsic motivation might help overcome these challenges. Thus, this case study highlights how difficult it is for retailers to go beyond the boundaries of the existing system of mass production and centralisation and that intrinsic motivations nevertheless help them to foster sustainably paths.
\end{abstract}


Teil III - Der Lebensmitteleinzelhandel als Gestalter nachhaltiger Konsum- und

Produktionsmuster

\section{Introduction}

In the context of sustainability discourse, food production and consumption are becoming the focus of sociopolitical and scientific discussions (Willett et al., 2019). As it is located between consumers and farmers, the food retail trade may play an important role in facilitating a transition towards more sustainable food production and consumption. Globally, (Sexton \& Xia, 2018) and especially in Germany (Nielsen Tradedimensions, 2019), it is a highly concentrated industry that leads to strong purchasing power over suppliers (farmers). Thus, farmers depend on a few food retailers to sell their products. This dependency enables food retailers to enforce their own standards and subsequently influence the meat market through their own marketing activities (Peattie \& Peattie, 2009; Sands et al., 2009). More, concentration leads to strong price pressure that in turn fosters the structural change in food production, from many small producers to few large producers (Bui et al., 2019; Konefal et al., 2005). Path dependence and the stability of the existing system make it difficult for radical sustainability innovations to emerge in food retail (Esbjerg et al., 2016; Geels, 2004).

Based on their organizational structure food retail cooperatives might play an important role in a transition towards more sustainable food consumption and production. They differ from conventional food retailers as such they benefit from joint advertising and promotion efforts while having the freedom to make independent decisions regarding their product range which enables them to initiate alternative paths. Examples are Germany's largest food retailer, Edeka Group; France's E.Leclerc and Système U; or New Zealand's Foodstuffs. Recently, food retail cooperatives in Europe have already aimed to foster an alternative path that deviates from the existing aim to centralise food retailing; as such, they have increasingly initiated the marketing of local food products (Bui et al., 2019) and thus, the collaboration with local farmers. In Germany in particular, several initiatives have been initiated (e.g. Edeka Group: 'Unsere Region-Gutes von Hier', Rewe Group: 'Aus deiner Region'). The resulting shorter supply chains are no longer based on mass production (quantity), but on more sustainable production (quality), which offers environmental, economic, and social advantages (Mastronardi et al., 2015; Pearson et al., 2011). Moreover, they are characterised by a limited number of economic operators as well as geographical proximity between farmers and consumers (Kneafsey et al., 2013). Thus, they reconnect food production and consumption, thereby fostering a more transparent way of food production and consumption (Vitters $\varnothing$ et al., 2019).

It can be assumed that deviating from existing supply chain mechanisms is accompanied by initial hurdles that require additional efforts by retailers. Retailers must invest time in 
Teil III - Der Lebensmitteleinzelhandel als Gestalter nachhaltiger Konsum- und

developing new marketing and logistical conditions (e.g. price negotiations, recovering unsold products, paying for supply disruption) that consider the perspective of small local farms, which are often unable to face retailers' high demands (Bui et al., 2019). As consumers need to be informed about the advantages of the locally produced food and the low proximity to farmers, retailers must also rework their existing marketing and communication concepts. Thus, retailers' investment is accompanied by uncertainty and additional organisational as well as financial efforts. As the marketing of niche products is usually accompanied by a low initial sales performance (Geels, 2004), it can be assumed that financial incentives play a subordinate role here. However, currently, the adaption process of food retailers is not entirely understood.

The multi-level perspective on social-technical transitions serves as a possible framework to describe a change toward more sustainable food production and consumption (Lachman, 2013). According to this approach, transitions occur through interaction within and between niches, regimes, and a sociotechnical landscape. Niches are considered the locus of innovations, where niche pioneers find a way to develop alternative approaches (Bilani, 2019; Geels, 2004). Regimes are described as incumbent sociotechnical systems (Holtz et al., 2008), and landscapes form an exogenous environment (e.g. cultural patterns) (Geels \& Schot, 2007) that put pressure on regimes and create opportunities for niches (Bilani, 2019). A successful transition is defined as a shift from one existing regime to another.

Applying these definitions to food retailer initiating the marketing of local food the existing high centralisation and standardisation in conventional food retailing can be described as the regime system (Bilani, 2019). Trends such as increasing public interest in how and where meat is produced can be described as the landscape environment (Bilali, 2019; Immink et al., 2013). Increased landscape pressure in turn create opportunities for retailers to initiate cooperations with local farmers. Until now, such alternative marketing approaches remain in niches while the existing regime (food retail) mainly focuses on centralisation. Geels and Schot (2007) distinguished between different transition pathways depending on the timing and nature of multi-level interactions. Regarding food retailers' impact on transitioning towards more sustainable food production and consumption, the transformation pathway is of particular interest. It describes insufficiently developed niche innovations occurring parallel to moderate landscape changes. These in turn create pressure on regime actors who react by modifying the direction of future development while the basic regime architecture remains the same (Geels \& Schot, 2007). A well-known example describing the transformation pathway is the development of organic food that was initiated by green activists in niches and later picked up by food retailers (regime actors) (Geels \& Schot, 2007; Smith, 2006). 
Teil III - Der Lebensmitteleinzelhandel als Gestalter nachhaltiger Konsum- und

Until now, sustainable transition research tended to overlook agro-food systems (Bilani, 2019). But based on health, animal welfare, and environmental concerns, scientists as well as consumers demand more sustainable production and consumption of livestock products and, especially meat products (Aleksandrowicz et al., 2016; Busch et al., 2018; Godfray et al., 2018; Willett et al., 2019). Thus, a deeper understanding of a sustainability transition in the meat market is of considerable interest. Until now, the German meat market has concentrated more on mass-produced rather than sustainably produced meat. Most meat products are advertised according to their low price rather than their quality, emphasising a low market differentiation. Therefore, the German meat market is characterised by its adherence to the strategy of cost leadership rather than by a focus on quality differentiation (Porter, 1980). Locally produced meat offers a possibility to foster a sustainable product differentiation on the meat market. As the main meat source for German consumers (Kohlmüller \& Koch, 2018) food retailers are considered as key actors in moving towards or away from sustainable production and consumption patterns of meat. However, until now they have been mostly neglected in recent transition publications. Thus, a deeper understanding of food retailers' product range decision regarding locally produced meat is of considerable importance in order to foster a sustainable transition in meat production and consumption patterns.

Drawing on this theoretical background, this study adopted an explorative mixed-method approach to investigate food retailers' perceived challenges and motives in marketing locally produced meat. Specifically, we used a case study of locally produced grass-fed beef marketed by Germany's leading food retail cooperative. The results of this study contribute to a stronger understanding of why some food retailers deviate from the industry status quo of standardisation, centralisation and low quality differentiation in the meat market while others continue to go ahead with the existing marketing approach.

\section{Case Study}

This study involved Germany's leading food retail cooperative. The cooperative has three management levels and consists of a national head office, seven regional wholesalers, and about 3,700 affiliated retailers. The retailers that participated in this study were from the cooperative's second-largest regional wholesaler, which is responsible for marketing food in the south of Germany and has a total of 1,300 grocery stores. Because of its cooperative structure, 951 of these retailers are owner-managed, i.e. each owner can make his own decisions regarding the product ranges he stocks and benefits from the cooperative's promotional activities. Further, consultants employed by the company support food retailers with their marketing and 
Teil III - Der Lebensmitteleinzelhandel als Gestalter nachhaltiger Konsum- und

communication activities. In addition to the legally independent cooperative members, the remaining retailers are managed by the regional wholesaler, but local shop management is still able to make some decisions regarding stocking the grass-fed beef examined in this study. ${ }^{1}$

Because of the regional purchasing power in the south of Germany, the regional wholesaler can focus on high-quality meat products. One sustainably produced product that some of the retailers already sell is locally produced grass-fed beef. The beef from southern Germany is produced in natural conservation areas during summer from local farmers. These areas are hilly and otherwise not suitable for agriculture. Outside the growing season, cattle are held in stables. The smallholding and less standardised farm structure lead to a low level of genetics standardisation, i.e. farmers use a wide variety of breeds. Because of seasonal calving and the fact that the number of cattle exceeds the availability of stable places, beef supply is characterised by seasonal variations ${ }^{2}$. However, the animals are able to graze outside in the summer and are free to express their normal behaviour; this farming method therefore fulfils the public's demands for animal welfare (Borkfelt et al., 2015). Additionally, this extensive grazing maintains the traditional aesthetic of the countryside (Barrantes et al., 2009) and promotes biodiversity (Rook \& Tallowin, 2003). Further, the elaborate production process is organically certified. Thus, retailers who want to sell the product need organic certification, too (GfRS, 2012). Currently, 115 retailers from the retail cooperative already sell locally produced grass-fed beef, and 92 of these are independent. Retailers can either buy the beef from the regional wholesaler or make their own contracts with farmers. ${ }^{3}$

\section{Methodology}

This study adopted a mixed-method approach. The combination of different sampling methods is viewed as preferable because it provides a better understanding of the research question (Palinkas et al., 2011). As food retailers' initial challenges and motives in marketing local food have rarely been investigated before, conducting qualitative interviews with consultants and retailers offered an opportunity to obtain new insights into their experiences and opinions (Pullman \& Dillard, 2010; Rombach \& Bitsch, 2015). The context provided by the qualitative interviews could then be used to devise the subsequent quantitative survey to confirm and further explain the results from the interviews. For both, the qualitative interviews and the quantitative survey all participants were part of the regional wholesaler. Retailers who already

\footnotetext{
${ }^{1}$ Based on an informal dialogue with the cooperative during this investigation.

${ }^{2}$ Based on an informal dialogue with the cooperative and farmers during this investigation.

${ }^{3}$ Based on an informal dialogue with the cooperative and farmers during this investigation.
} 
Teil III - Der Lebensmitteleinzelhandel als Gestalter nachhaltiger Konsum- und

sold the product and retailers who did not were asked to take part. The participating consultants also advised retailers that already stocked grass-fed beef and those that did not. All participating retailers were in a position to make independent decisions about adding grass-fed beef to their range. This selection of participants created a balanced picture of the challenges and motives involved in marketing grass-fed beef. The study was supported by the regional wholesaler who provided contact details for participants in the qualitative interviews and passed the link on to the retailers for the quantitative online survey.

In June 2018, 12 qualitative interviews were conducted: 10 with retailers and two with consultants. The interviews with consultants were conducted with the assumption that they possessed a broad understanding of marketing grass-fed beef. As these consultants were in contact with multiple retailers. Including them in this study ensured the identification of a wide range of challenges and motives, which could thus improve the development of the quantitative survey. The interviews took place over the phone and followed a semi-structured format. On average, each interview lasted 20 minutes. All interviews were recorded. After data acquisition, the interviews were transcribed and analysed using qualitative content analysis according to Mayring (2010) using MAXQDA Plus 2020. The following code categories were determined deductively (Table 1): 'experience in the marketing of grass-fed beef'; 'challenges in marketing' with the inductively identified sub-categories of 'supply chain-related' and 'marketing-related'; and 'motives in marketing' with the inductively identified sub-categories of 'intrinsic motivation (values and attitudes)' and 'extrinsic motivation (financial incentives)'. 
Teil III - Der Lebensmitteleinzelhandel als Gestalter nachhaltiger Konsum- und

Table 1. Code Categories

\begin{tabular}{llll}
\hline Main category & Sub-category & Approach & Source \\
\hline Experience & - & deductive & $\begin{array}{l}\text { Personal briefing with regional } \\
\text { wholesaler }\end{array}$ \\
Challenges & Supply chain-related & deductive / inductive & Schulze \& Risius (2019) \\
& Marketing-related & deductive / inductive & Schulze \& Risius (2019) \\
Motives & $\begin{array}{l}\text { Extrinsic (financial } \\
\text { incentives) }\end{array}$ & deductive & Papagiannakis \& Lioukas (2012) \\
& $\begin{array}{l}\text { Intrinsic (values and } \\
\text { attitudes) }\end{array}$ & deductive / inductive & $\begin{array}{l}\text { Schulze et al. (2019), } \\
\text { Papagiannakis \& Lioukas (2012) }\end{array}$ \\
\hline
\end{tabular}

Subsequently, based on the results of the qualitative interviews, a standardised survey was developed to conduct the online quantitative survey from March until June 2019. Item development and adaption took place based on the results of the qualitative interviews and considered supply chain as well as marketing-related challenges. The survey comprised three different parts. First, retailers were asked about several characteristics of their market (e.g. number of employees, sales area) and their past and future product-range decisions regarding grass-fed beef. Second, retailers were asked about the challenges of marketing grass-fed beef. Third, based on the results of the qualitative interviews, the retailers' extrinsic and intrinsic motives were investigated. Intrinsic motives were investigated by differentiating between values and attitudes, which is a common approach in empirical research (e.g. Papagiannakis \& Lioukas, 2012). Values were examined according to Schwartz's theory of values (1992). However, based on results from Papagiannakis and Lioukas (2012), only the values of selftranscendence and self-enhancement were considered. At the attitude level, the retailers were questioned about their awareness of animal welfare based on the animal treatment scale developed by Kendall et al. (2006) and two items by Schulze et al. (2019). A detailed description of the items used is shown in Table 2. To illustrate the external financial incentives, a single item, 'When selling locally produced grass-fed beef, financial success is most important', was adapted from Papagiannakis and Lioukas (2012).

In total, 57 retailers took part in the survey. Data checks for quality showed no conspicuous response behaviour (inconsistencies, straightlining, or responses given too fast). The quantitative data were analysed using IBM SPSS Statistics 26 and RStudio 1.2.5003. As explorative factor analysis can yield reliable results even for small sample sizes (de Winter et al., 2009), a principal component analysis (PCA) was used to identify underlying theoretical challenges and motives. Subsequently, retailers were grouped according to their past and future 
Teil III - Der Lebensmitteleinzelhandel als Gestalter nachhaltiger Konsum- und

product-range decisions regarding locally produced grass-fed beef. However, two retailers already stocked locally produced grass-fed beef but did not plan to stock it in the future. As this group was too small for further statistical analysis, these two retailers were excluded (see Appendix 2). Therefore, 55 respondents were used for the final data analysis. Groups were thus described according to the challenges and motives obtained from the PCA. Although an analysis of variance (ANOVA) was applied to detect differences between groups, this study mainly focused on mean and standard deviations to derive tendencies. This was appropriate because of the small sample size.

\section{Results}

\section{Qualitative Results}

Seven out of the ten retailer interviewees already stocked locally produced grass-fed beef; one said he was among the initiators of the marketing program; two said they wanted to concentrate exclusively on marketing the grass-fed beef and tried to stock conventional beef only as an exception. The remaining three retailer interviewees did not stock the locally produced grassfed beef at the time of the interview. However, two imagined that they could stock it at some point in the future. One had removed it from his product range and did not imagine that it would be reintroduced at any time. The two interviewed consultants stated that about half of the grocery stores under their supervision already stocked locally produced grass-fed beef.

Regarding supply chain-related challenges, the interviewees complained about too long order periods, which required considerable planning and led to increased uncertainty. In addition, the interviewees were not able to reorder single cuts of the product, which created a surplus of locally produced grass-fed beef or limited product availability for customers depending on the retailers' purchasing behaviour. The interviewees also named challenges caused by differences in product quality. The main reason for quality differences was a lack of product standardisation:

The producers should sit down together and find some sort of standard. Both in breeds and feed, so that we get more standardised. The product differs too much from producer to producer and from animal to animal. $(L, 35)$

The main marketing-related hurdle was the additional communication effort needed to inform customers about the product's elaborate production process. The interviewees stated that direct 
Teil III - Der Lebensmitteleinzelhandel als Gestalter nachhaltiger Konsum- und

contact with their customers was very important. This involved making the staff aware of the locally produced grass-fed beef's added value:

It is always important that the personnel know about the product and can answer questions. Where does the meat come from? How are the animals reared? What type offarming is practised? $(C, 48)$

The retailer interviewees said that additional staff training improved their marketing. They were aware of how vital the staff-customer interaction is and attempted to build the knowledge and enthusiasm of their staff (e.g. by organising trips to local farms). However, they were aware of the additional effort accompanied by training their staff. Further, having a lack of qualified staff was perceived as a difficulty in marketing locally produced grass-fed beef.

Another marketing-related hurdle was promoting the less popular parts of an animal. Usually, consumers prefer prime cuts (e.g. fillet). As it was not possible to reorder single cuts, retailers were confronted with the challenge of how to market less popular parts (e.g. stewing meat) without having a product surplus or shortage. However, some of the interviewees highlighted possible solutions. This especially applied to retailers who already had experience with the marketing of locally produced grass-fed beef. One thought that the problems regarding reordering smaller cuts could be solved if more retailers added locally produced grass-fed beef to their product range. Others stated that they had found their own solution to the problem by processing secondary cuts into convenience products (e.g. cooked goulash to warm up at home).

Both extrinsic and intrinsic motivations can be identified as drivers to invest in the marketing of locally produced grass-fed beef. One extrinsic motive named was the growing public interest in animal welfare issues. However, just a few of the interviewees mentioned financial incentives as the main driver for the investment in the marketing of locally produced grass-fed beef.

Intrinsic motives were named more often. Interviewees highlighted the elaborate production process that they related to a high degree of animal welfare and environmental protection. Thus, they named pasture access, grass-fed diets, and freedom to express normal behaviour and its implications for sensory product characteristics (e.g. taste, marbling) as advantages of grassfed beef. Further, they appreciated the farmers' work and wanted them to be compensated. The local production process was perceived as beneficial both for supporting local farmers and for bringing together farmers and consumers. 
Teil III - Der Lebensmitteleinzelhandel als Gestalter nachhaltiger Konsum- und

I am a fan of the meat myself. I see for myself where the cows graze. I must admit

that I wasn't able to transfer this [feeling] to my sales team until I had seen it on

the farm, with the farmers, with the cows in the fields. $(B, 87)$

\section{Quantitative Results}

Retailer representatives from the regions of Baden-Wuerttemberg (52.7\%), RhinelandPalatinate (20.0\%), Saarland (1.8\%), Hesse (21.8\%) and Bavaria (3.6\%) took part in the survey, thereby representing the regional wholesaler's entire catchment area. More than half of these retailer representatives owned one grocery store $(56.6 \%)$. The remaining retailer representatives owned more than one store (43.4\%). Most had fewer than 100 employees (74.0\%), while the rest had more than 100 employees (26.0\%). Most of the grocery stores owned by retailers covered an area between 1,001 and 2,500 $\mathrm{m}^{2}(75.5 \%)$; the remaining stores were smaller $(14.3 \%)$ or larger $(10.2 \%)$. Nearly half of the retailers were over 40 years old $(48.9 \%)$, and most of the retailer representatives were male (69.1\%) (see Appendix 1).

PCA was used to detect the underlying challenges and motives in marketing locally produced grass-fed beef (Table 2).

Table 2. Results of Principal Component Analysis

\begin{tabular}{llll}
\hline Item & Description & Mean (SD) & $\begin{array}{l}\text { Factor } \\
\text { Loading }\end{array}$ \\
\hline
\end{tabular}

Challenges $1($ KMO $=.53)$

Supply chain-related challenges $(\mathrm{CA}=.72)$

$2.85(0.95)$

Ch_SC1 Variations in quality

$3.04(1.33) \quad 0.87$

Ch_SC2 Seasonal availability

$2.96(1.21) \quad 0.63$

Ch_SC3 Sub-standard quality

$2.67(1.45) \quad 0.78$

Ch_SC4 Inadequate market supply

$2.77(1.14) \quad 0.60$

Marketing-related challenges $(\mathrm{CA}=\mathbf{0 . 7 0})$

$3.35(0.93)$

Ch_MA1 Organic certification

$3.27(1.15) \quad 0.79$

Ch_MA2 Training employees

$3.19(1.18) \quad 0.85$

Ch_MA3 Marketing secondary cuts

3.65 (1.17) $\quad 0.70$

Values $^{2}($ KMO $=.63)$

Self-transcendence $(\mathrm{CA}=\mathbf{0 . 7 1})$

$4.18(0.56)$

SV_ST1 It is important to him to listen to people who are different $3.95(0.95)$ from him. Even when he disagrees with them, he still wants to understand them.

SV_ST2 He strongly believes that people should care for nature. $4.22(0.71) \quad 0.64$ Looking after the environment is important to him. 

should be treated equally. He believes everyone should have equal opportunities in life.

SV_ST4 It is very important to help the people around him. He $\quad 4.20(0.80) \quad 0.71$ wants to care for their wellbeing.

SV_ST5 It is important to him to be loyal to his friends. He wants $4.64(0.56) \quad 0.53$ to devote himself to people close to him.

Self-enhancement $(\mathrm{CA}=\mathbf{. 6 6})$

SV_SE1 It is important to him to be rich. He wants to have a lot of 2.60 (1.13) money and expensive things.

SV_SE2 It is important to him to get respect from others. He wants $3.84(0.81) \quad 0.46$ people to do what he says.

SV_SE3 Being very successful is important to him. He hopes $3.91(0.98) \quad 0.80$ people will recognise his achievements.

SV_SE4 It is important to him to show his abilities. He wants 3.44 (1.05) 0.75 people to admire what he does.

Attitude $^{3}$

Animal welfare awareness $(\mathrm{CA}=\mathbf{. 6 9})$

$4.13(0.64)$

AW1

How animals in livestock production are housed is

$4.49(0.66)$

0.80 important to me.

AW2

Livestock farming raises serious ethical questions about

$4.11(0.82)$

AW3 the treatment of animals.

I don't care how animals are treated in livestock

$4.51(0.92)$

0.78 production.

AW4

Regulation of the treatment of animals in farming is

$3.48(1.11)$

0.70 sufficient.

$\mathrm{KMO}=$ Kaiser-Meyer-Olkin. $\mathrm{CA}=$ Cronbach's Alpha. ${ }^{1}$ On a scale of $1=$ absolutely unproblematic to $5=$ extremely problematic. ${ }^{2}$ On a scale of $1=$ not like me at all to $5=$ very much like me. ${ }^{3}$ On a scale of $1=$ not true at all to $5=$ absolutely true. AW3 and AW4 were recoded.

Source: Own calculations.

Confirming the results from the qualitative interviews, the first PCA on challenges identified two factors: 'supply chain-related challenges' and 'marketing-related challenges'. The item 'length of ordering process' was excluded because of low factor loadings. The factor 'supply chain-related problems' refers to variations in quality, seasonal availability, inadequate market supply, and sub-standard quality. Variations in quality were perceived as problematic by 43.4 $\%$ of the questioned retailer representatives, seasonal availability by $41.5 \%$, inadequate market supply by $28.3 \%$, and sub-standard quality by $30.5 \%$. The factor 'marketing-related challenges' refers to marketing secondary cuts, organic certification, and training employees. Marketing secondary cuts - those that are less attractive to consumers but that must also be marketed-was perceived as problematic by $53.9 \%$ of the retailer representatives, organic 
Teil III - Der Lebensmitteleinzelhandel als Gestalter nachhaltiger Konsum- und

certification by $45.1 \%$, and training employees by $46.3 \%$. Overall, supply-chain challenges were perceived as less problematic $(\mu=2.85)$ than marketing challenges $(\mu=3.35)$.

The two underlying concepts of 'self-transcendence' and 'self-enhancement', as developed by Schwartz (1992), were identified by the second PCA. Both values showed a medium to high manifestation level. However, the retailer representatives identified more strongly with the value of 'self-transcendence' ( $\mu=4.18)$ than with 'self-enhancement' $(\mu=3.45)$.

Based on the third PCA, the items describing the retailer representatives' perceptions of livestock production can be summarised by the factor 'animal welfare awareness'. The item 'Generally, people show too little respect for animals' was excluded because of insufficient factor loading. Overall, the sample demonstrated high awareness of the welfare of farm animals $(\mu=4.13)$. Financial incentive was perceived as somewhat important in marketing grass-fed beef $(\mu=3.33)$. Thus, $40.0 \%$ of the retailer representatives agreed with the item 'When selling grass-fed beef, financial success is most important', and $20.0 \%$ disagreed. The remaining 20.0 $\%$ were unsure.

Based on the retailer representatives' past and future stocking decisions regarding locally produced grass-fed beef, the retailers were grouped and subsequently described according to their perceptions of supply chain-related and marketing-related challenges, and according to their intrinsic (self-enhancement, self-transcendence, animal welfare awareness) and extrinsic (financial incentive) motives. 22 retailers did not stock the product and did not plan to do so in the near future. This group was named 'opponents'. 18 retailers did not stock the product but planned to do so. This group was named 'niche prospects'. 15 retailers already sold the grassfed beef and wanted to continue selling it in the near future. This group was named 'niche pioneers'. 
Figure 1. Descriptives by Group

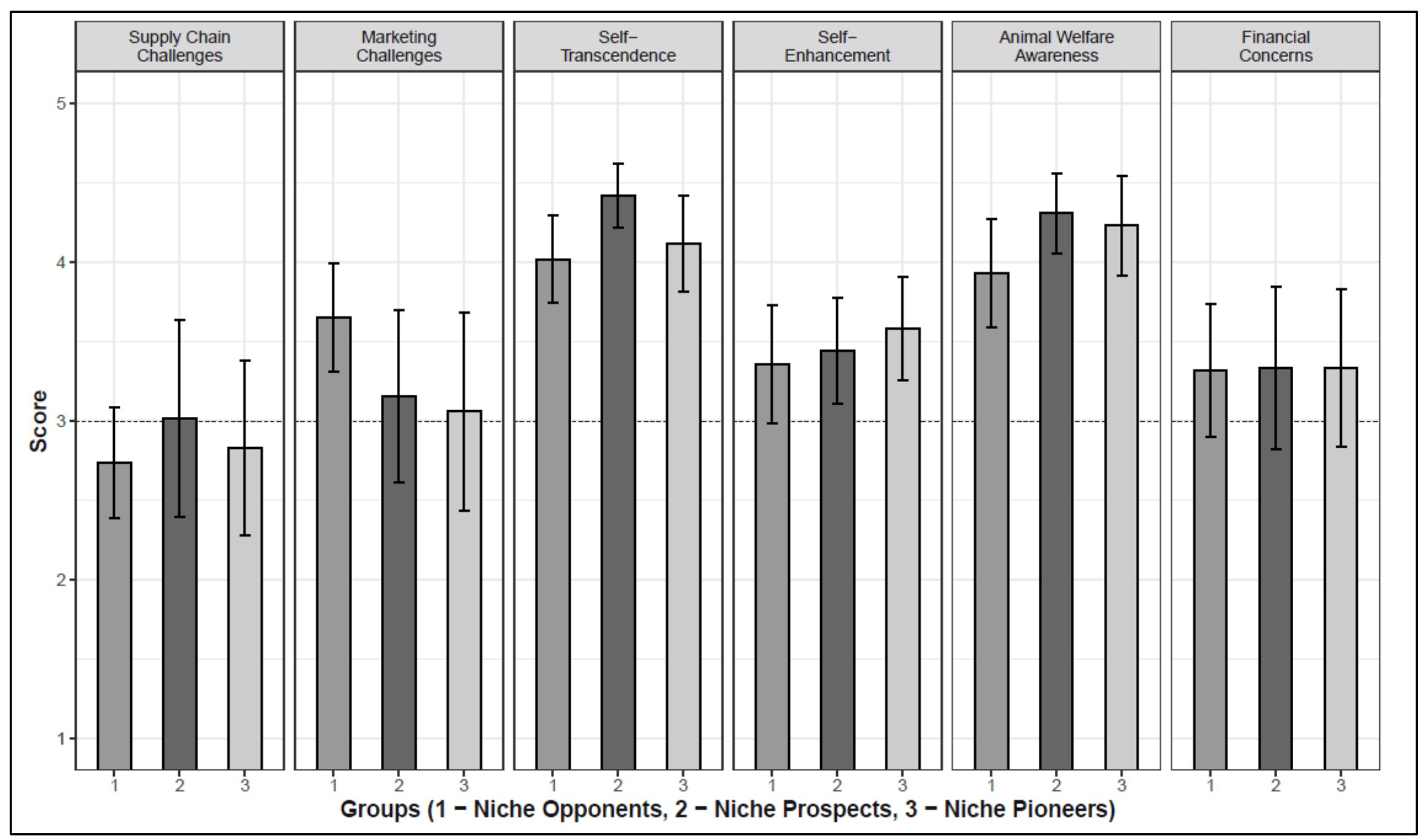

Ch $\_$MA\&Ch $\_$SC on a scale from $1=$ absolutely unproblematic to $5=$ extremely problematic. SV_ST\&SV_SE on a scale from $1=$ not like me at all to $5=$ very much like me. AW \& Financial Concern on a scale from $1=$ not true at all to $5=$ totally true. Differences according to ANOVA: Ch_MA: $\mathrm{p}=.1271 ; \mathrm{Ch} \_$PC: $\mathrm{p}=.3017 ; \mathrm{SV} \_\mathrm{ST}$ : $\mathrm{p}=.2243$, SC_SE: $\mathrm{p}=.6958 ; \mathrm{AW}: \mathrm{p}=.0911 ;$ Financial Concern: $\mathrm{p}=.7115$. 
Teil III - Der Lebensmitteleinzelhandel als Gestalter nachhaltiger Konsum- und

Niche opponents perceived supply chain-related challenges as somewhat problematic $(\mu=2.74$, $\mathrm{SD}=0.76$ ). Thus, compared with the other groups, niche opponents perceived these challenges as less problematic. In contrast, they perceived marketing-related challenges as the most problematic $(\mu=3.65, \mathrm{SD}=0.75)$. Regarding intrinsic motives, niche opponents somewhat agreed with self-enhancement $(\mu=3.36, \mathrm{SD}=0.82)$, but had the lowest agreement with this value compared with the other groups. Their agreement with self-transcendence was higher $(\mu$ $=4.02, \mathrm{SD}=0.62$ ), but this group identified with self-transcendence the least compared with the other groups. This was also the case for their agreement with animal welfare awareness: while niche opponents had a strong interest in animal welfare $(\mu=3.93, \mathrm{SD}=0.77)$, they had the lowest interest compared with the other groups. They considered financial success somewhat important $(\mu=3.32, \mathrm{SD}=0.95)$; all three groups showed a similar level of agreement with this motive.

Niche prospects had the highest mean for supply chain-related challenges $(\mu=3.02, \mathrm{SD}=1.16$ ), but perceived marketing-related challenges as more problematic. However, compared with niche opponents, niche prospects perceived marketing-related obstacles as less problematic. Regarding intrinsic drivers, niche prospects identified more strongly with self-enhancement $(\mu$ $=3.44, \mathrm{SD}=0.67)$ than opponents. The same applied for self-transcendence $(\mu=4.42, \mathrm{SD}=$ 0.41). However, this group's identification with self-transcendence was the highest of all three groups. This was also the case for animal welfare awareness $(\mu=4.31, \mathrm{SD}=0.49$ ). Financial success was somewhat important for niche prospects $(\mu=3.33, \mathrm{SD}=1.03)$.

Pioneers perceived supply chain-related challenges as somewhat problematic $(\mu=2.83, \mathrm{SD}=$ 0.99), but as less problematic than niche prospects and more problematic than opponents. Pioneers also viewed marketing-related challenges as somewhat problematic $(\mu=3.06, \mathrm{SD}=$ 0.93), but as less problematic than niche prospects and opponents. Niche pioneers had the highest identification with self-enhancement $(\mu=3.58, \mathrm{SD}=0.59)$. Self-transcendence $(\mu$ $=4.12, \mathrm{SD}=0.54)$ and animal welfare awareness $(\mu=4.23, \mathrm{SD}=0.54)$ was lower compared to niche prospects but higher compared to opponents. Financial success was somewhat important for niche pioneers $(\mu=3.33, \mathrm{SD}=0.90)$.

\section{Discussion}

In recent times, retail cooperatives have been initiating alternative marketing approaches to sell local food products. Thus, they deviate from the existing approach of centralisation in the food retail industry (Bui et al., 2019). The case of locally produced grass-fed beef marketed by the 
Teil III - Der Lebensmitteleinzelhandel als Gestalter nachhaltiger Konsum- und

leading German food retail cooperative was used to investigate retailers' challenges and motives to initiate the marketing of local food. More, due to the elaborate production process, grass-fed beef is characterised by several sustainable product attributes regarding animal welfare, environmental, and health and social issues that help to foster quality differentiation at the meat market.

Our results showed that from a retailers' perspective, the supply chain and particularly the production process of locally produced grass-fed beef, is accompanied by a lack of standardisation that leads to quality variations as well as supply shortages. Retailers are used to the existing marketing approach of mass production that allows for standardised meat products and perceive deviations from the existing approach as challenging. Thus, this study provides evidence for the stability of the existing system (Esbjerg et al., 2016; Geels, 2004) that makes it difficult for innovations to emerge. This seems particularly true for the niche prospects' decision to introduce grass-fed beef, as this group perceived supply chain-related challenges as most problematic.

Further, our results showed that retailers who invest in marketing grass-fed beef are confronted with challenges that are normally faced by the meat industry. One example is the necessity of processing and marketing less popular meat cuts. Normally, retailers reorder single cuts to avoid shortages of popular meat cuts (e.g. fillet), and the meat industry takes care of less popular parts (e.g. via export). As consumers' cooking abilities as well as the time spent on cooking decline (McGowan et al., 2017), selling less popular parts (e.g. stewing meat) that need elaborate cooking skills and more time to be prepared is a particular challenge in the marketing of grassfed beef.

In addition, our results showed that the product needs additional communication efforts to show the added value to consumers. As consumers are not able to experience the elaborate production process before nor after consumption, they require additional information to understand the advantage of the product (Grunert et al., 2004). The German meat market is characterised by a low market differentiation and thus, retailers are used to seeing (fresh) meat as an unbranded product (Grunert, 2006). Hence, additional communication efforts are perceived as a challenge. As our results showed the perceived importance of customer interaction, retailers depend on educated and enthusiastic staff. Increasing skills shortage in Germany (Federal Labour Office Germany, 2019) has complicated the marketing of grass-fed beef. As additional communication efforts are perceived as most problematic by opponents followed by niche prospects, additional 
Teil III - Der Lebensmitteleinzelhandel als Gestalter nachhaltiger Konsum- und

marketing efforts seem to be an explanation for non-adoption of introducing sustainably produced beef.

More, qualitative as well as quantitative results indicated that financial incentives play a subordinate role in retailers' product range decision whether to sell grass-fed beef. Retailers seem to be aware of initial low sales performance (Geels, 2004) of niche products and do not invest in collaborations with local farmers to meet direct financial success. However, the present study did not investigate indirect financial success such as improved image. However, Bui et al. (2019) pointed to the consideration of retailers' interest in building a positive image on the value of solidarity when selling local food.

Intrinsic motives seem to support retailers' decision regarding the investment in the alternative marketing approaches. This result is in line with the findings of Gemünden et al. (2007), indicating that retailers' intrinsic motivation could explain their investment in alternative marketing approaches. Niche pioneers and opponents demonstrated higher identification with the concepts of self-transcendence and animal welfare awareness, confirming the results of Williams and Schaefer (2013), who highlighted the importance of key figures' intrinsic motivation in a company's sustainability orientation. Identifying with self-transcendence was higher for niche pioneers and niche prospects, aligning with the results of Papagiannakis and Lioukas (2012), who found that entrepreneurs' identification with self-transcendence increases their pursuance of sustainability goals.

\section{Practical Implications}

Some practical implications can be drawn from this study's key findings to improve local niche marketing and thus the transformation towards a more sustainable meat production and consumption: As marketing-related challenges were perceived as most problematic, particular attention should be paid to them. Retailer should be given access to information about how to meet these initial marketing challenges. Solutions developed by niche pioneers should be used as models to motivate niche prospects and opponents. Consultants could help spread this information, as they are in contact with a wide range of different retailers. However, at the same time, supply chain-related problems should also be solved. Retailers and farmers should jointly discuss further developments and solutions regarding initial challenges such as how to cope with variations in quality or seasonal product availability. More, some retailers already found solutions for how to contend with processing and marketing secondary cuts of meat (e.g. by processing secondary cuts into convenience products) that could act as a model for others. As not every retailer is able to implement such practices, cooperatively organised processing of 
Teil III - Der Lebensmitteleinzelhandel als Gestalter nachhaltiger Konsum- und

less popular parts may be a solution. In addition, retailers could use perceived supply chainrelated challenges as opportunities. For example, they could highlight variable product characteristics based on the less standardised production process (e.g. fat content) to demonstrate the speciality of the product. Furthermore, variations in seasonal availability could be used to enhance demand in specific seasons. This marketing approach is already known from strawberry or asparagus and could be used in the marketing of alternatively produced meat, as well. Additionally, the results of this study emphasise the strong influence of key figures' intrinsic motivation, suggesting that cooperatives that plan to foster more sustainable food retailing should consider values and attitudes in their selection process.

\section{Conclusion}

Retailers who introduced the marketing of locally produced grass-fed beef supported local farms that cannot meet the food retail industry's high demands for standardisation. By selling locally produced grass-fed beef, these retailers fostered trust and the connection between consumers and producers, which could improve understanding between these groups (Pearson et al., 2011). Moreover, the sense of being closely connected to the product and its production could lead to more sustainable meat consumption in the long run (Pearson et al., 2011).

It is therefore that this research concludes that an increasing landscape pressure (increasing interest in animal welfare issues, environmental concerns) could lead to more retailers picking up the marketing of sustainably produced meat products. Path dependence and the stability of the existing system, however, will make immediate regime changes more difficult (Geels, 2004; Geels \& Schot, 2007). Although working with local farmers increases retailers' reputation, consumers are used to low prices when buying fresh meat. Thus, retailers are confronted with the challenge to find the balance between supporting sustainably produced meat products, accompanied by higher prices, while not provoking to lose customers. For this reason, a gradual adjustment of regime rules according to the transformation pathway seems more realistic (Geels $\&$ Schot, 2007). The existing regime rules in food retailing (centralisation, standardisation) are likely to survive while alternative approaches such as food retailers' collaboration with local farmers gradually adjust regime rules (Geels \& Schot, 2007).

Acknowledgements: This work was supported by the Federal Office for Agriculture and Food, Germany (BLE 2818301416) and the Ministry of Science and Arts (MWK) in Lower Saxony, Germany. The authors are responsible for the content of this publication. 
Teil III - Der Lebensmitteleinzelhandel als Gestalter nachhaltiger Konsum- und

\section{References}

Aleksandrowicz, L., Green, R., Joy, E. J. M., Smith, P., \& Haines, A. (2016). The impacts of dietary change on greenhouse gas emissions, land use, water use, and health: A systematic review. PLoS ONE, 11(11), e0165797.

Barrantes, O., Ferrer, C., Reine, R., \& Broca, A., (2009). Categorisation of grazing systems to aid the development of land use policy in Aragon, Spain. Grass and Forage Science, 64(1), 26-41.

Bilani, E. H. (2019). The multi-level perspective in research on sustainability transitions in agriculture and food systems: A systematic review. Agriculture, 9(74), 1-24.

Borkfelt, S., Kondrup, S., Röcklinsberg, H., Bjørksahl, K., \& Gjerris, M. (2015). Closer to nature? A critical discussion of the marketing of 'ethical' animal products. Journal of Agricultural and Environmental Ethics, 28(6), 1053-1073.

Bui, S., Costa, I., De Schutter, O., Dedeurwaerdere, T., Hudon, M., \& Feyereisen, M. (2019). Systemic ethics and inclusive governance: Two key prerequisites for sustainability transitions of agri-food systems [Special issue]. Agriculture and Human Values, 36(2), $277-288$.

Busch, G; Gauly, M., \& Spiller, A. (2018). What needs to be changed for successful future livestock farming in Europe? Animal, 12(10), 1999-2001.

de Winter, J. C. F., Dodou, D., \& Wieringa, P. A. (2009). Exploratory factor analysis with small sample sizes. Multivariate Behavioral Research, 44(2), 147-181.

Esbjerg, L., Burt, S., Pearse, H., \& Glanz-Chanos, V. (2016). Retailers and technology-driven innovation in the food sector: Caretakers of consumer interests or barriers to innovation? British Food Journal, 118(6), 1370-1383.

Federal Labour Office Germany (2019). Fachkräfteengpassanalyse Dezember 2019. https://statistik.arbeitsagentur.de/Navigation/Footer/TopProdukte/Fachkraefteengpassanalyse-Nav.html

Geels, F. W. (2004). From sectoral systems of innovation to socio-technical systems: Insights about dynamics and change from sociology and institutional theory. Research Policy, 33(6-7), 897-920.

Geels, F. W., \& Schot, J. (2007). Typology of sociotechnical transition pathways. Research Policy, 36(3), 399-417.

Gemünden, H. G., Salomo, S., \& Hölzle, K. (2007). Role models for radical innovations in times of open innovation. Creativity and Innovation Management, 16(4), 408-421. 
Teil III - Der Lebensmitteleinzelhandel als Gestalter nachhaltiger Konsum- und

GfRS (Gesellschaft für Ressourcenschutz) (2012). Zertifiziert Bio- erfolgreich im Einzelhandel. https://www.gfrs.de/fileadmin/files/gfrs_broschuere_biozertifizierung_ einzelhandel.pdf

Godfray, H. C. J., Aveyard, P., Garnett, T., Hall, J. W., Key, T. J., Lorimer, J., Pierrehumbert, R. T., Scarborough, P., Springmann, M., \& Jebb, S. A. (2018). Meat consumption, health, and the environment. Science, 361(6399), eaam5324.

Grunert, K.G. (2006) Future trends and consumer lifestyles with regard to meat consumption. Meat Science, 74(1), 149-160.

Grunert, K.G., Bredahl, L. \& Brunsø, K. (2004). Consumer perception of meat quality and implications for product development in the meat sector - a review. Meat Science, 66(2), 259-272.

Holtz, G., Brugnach, M., \& Pahl-Wostl, C. (2008). Specifying 'regime'-A framework for defining and describing regimes in transition research. Technological Forecasting and Social Change, 75(5), 623-643.

Immink, V. M., Reinders, M. J., van Tulder, R. J. M., \& van Trijp, J. C. M. (2013). The livestock sector and its stakeholders in the search to meet the animal welfare requirements of society [Special issue]. Journal on Chain and Network Science, 13(2), $151-160$.

Kendall, H. A., Lobao, L. M., \& Sharp, J. S. (2006). Public concern with animal well-being: Place, social structural location, and individual experience. Rural Sociology, 71(3), 399-428.

Kneafsey, M., Venn, L., Schmutz, U., Balázs, B., Trenchard, L., Eyden-Wood, T., Bos, E., Sutton, G. \& Blackett, M. (2013). Short Food Supply Chains and Local Food Systems in the EU. A State of Play of Their Socio-Economic Characteristics; European Commision: Brussels, Belgium.

Kohlmüller, M., \& Koch, T. (2018). Markt Bilanz-Vieh und Fleisch, ed. In AMI (Argrarmarkt Informationsgesellschaft). Medienhaus Plump GmbH.

Konefal, J., M. Mascarenhas, \& M. Hatanaka. (2005). Governance in the global agro-food system: Backlighting the role of transnational supermarket chains. Agriculture and Human Values, 22(3), 291-302.

Lachman, D. A. (2013). A survey and review of approaches to study transitions. Energy Policy, 58(July 2013), 269-276. 
Teil III - Der Lebensmitteleinzelhandel als Gestalter nachhaltiger Konsum- und

Mastronardi, L., Marino, D., Cavallo, A., \& Agostino, G. (2015). Exploring the role of farmers in short food supply chains: the case of Italy. International Food and Agribusiness Management Review, 18(2), 109-130.

Mayring, P. (2010). Qualitative Inhaltsanalyse-Grundlagen und Techniken. Beltz.

McGowan, L., Caraher, M., Raats, M., Lavelle, F., Hollywood, L., McDowell, D., Spence,... \& Dean, M. (2017). Domestic cooking and food skills: A review. Critical Reviews in Food Science and Nutrition, 57(11), 2412-2431.

Nielsen Trade Dimensions (2019). Top-Firmen Edition 2019. Aus: Fleischwirtschaft.de (2019). Die Top 5 bleiben an der Spitze. https://www.fleischwirtschaft.de/wirtschaft/charts/Lebensmittelhandel-Die-Top-5bleiben-an-der-Spitze-40682.

Palinkas, L. A., Aarons, G. A., Horwitz, S. M., Chamberlain, P., Hurlburt, M., \& Landsverk, J. (2011). Mixed method designs in implementation research. Administration and Policy in Mental Health and Mental Health Services Research, 38(1), 44-53.

Papagiannakis, G., \& Lioukas, S. (2012). Values, attitudes and perceptions of managers as predictors of corporate environmental responsiveness. Journal of Environmental Management, 100(June 2012), 41-51.

Pearson, D., Henryks, J., Trott, A., Jones, P., Parker, G., Dumaresq, D., \& Dyball, R. (2011). Local food: Understanding consumer motivations in innovative retail formats. British Food Journal, 113(7), 886-899.

Peattie, K., \& Peattie, S. (2009). Social marketing: A pathway to consumption reduction? Journal of Business Research, 62(2), 260-268.

Porter, M. E. (1980). Competitive strategy: Techniques for analyzing industries and competitors. Free Press.

Pullman, M. E., \& Dillard, J. (2010). Value based supply chain management and emergent organizational structures. International Journal of Operations \& Production Management, 30(7), 744-771.

Rombach, M., \& Bitsch, V. (2015). Food movements in Germany: Slow food, food sharing, and dumpster diving. International Food and Agribusiness Management Review, 18(3), 1-23.

Rook, A. J., \& Tallowin, J. R. B. (2003). Grazing and pasture management for biodiversity benefit. Animal Research, 52(2), 181-189. 
Teil III - Der Lebensmitteleinzelhandel als Gestalter nachhaltiger Konsum- und

Sands, S., Oppewal, H., \& Beverland, M. (2009). The effects of in-store themed events on consumer choice decisions. Journal of Retailing and Consumer Services, 16(5), 386395.

Schulze, M., \& Risius, A. 2019. Warenverfügbarkeit als Hemmnis in der Vermarktung von Biorindfleisch—die Perspektive des Lebensmitteleinzelhandels. In: Mühlrath, D., Albrecht, J., Finckh, M. R., Hamm, U., Heß, J., Knierim, U. \& Möller, D. Innovatives Denken für eine nachhaltige Land- und Ernährungswirtschaft. Beiträge zur 15. Wissenschaftstagung Ökologischer Landbau, Kassel, 5. bis 8. März 2019. Verlag Dr. Köster.

Schulze, M., Spiller, A., \& Risius, A. (2019). Food retailers as mediating gatekeepers between farmers and consumers in the supply chain of animal welfare meat-Studying retailers' motives in marketing pasture-based beef. Food Ethics, 3(1-2), 41-52.

Schwartz, S. H. (1992). Universals in the content and structure of values: Theoretical advances and empirical tests in 20 countries. Advances in Experimental Social Psychology, 25, 1-65.

Sexton, R. \& Xia, T. (2018). Increasing Concentration in the Agricultural Supply Chain: Implications for Market Power and Sector Performance. Annual Review of Ressource Economics, 10(2018), 229-251.

Smith, A. (2006). Green niches in sustainable development: The case of organic food in the United Kingdom. Environment and Planning C: Government and Policy, 24(3), 439458.

Vittersø, G., Torjusen, H., Laitala, K., Tocco, B., Biasini, B., Csillag, P., de Labarre, M.D., ... \& Wavresky, P. (2019). Short Food Supply Chains and Their Contributions to Sustainability: Participants' Views and Perceptions from 12 European Cases. Sustainability, 11(17), 4800.

Willett, W., Rockström, J., Loken, B., Springmann, M., Lang, T., Vermeulen, S., Garnett, T., ... \& Murray, C. J. L. (2019). Food in the Anthropocene: The EAT-Lancet Commission on healthy diets from sustainable food systems. The Lancet, 393(10170), 447-492.

Williams, S., \& Schaefer, A. (2013). Small and medium sized enterprises and sustainability: managers' values and engagement with environmental and climate change issues. Business Strategy and the Environment, 22(3), 173-186. 
Teil III - Der Lebensmitteleinzelhandel als Gestalter nachhaltiger Konsum- und Produktionsmuster

\section{Appendix}

Appendix 1. Characteristics of Participating Retailers

\begin{tabular}{|c|c|c|}
\hline Characteristic & $\mathrm{N}$ & $\%$ \\
\hline \multicolumn{3}{|l|}{ Federal state $(n=55)$} \\
\hline Baden-Wuerttemberg & 29 & 52.7 \\
\hline Rhineland-Palatinate & 11 & 20.0 \\
\hline Saarland & 1 & 1.8 \\
\hline Hesse & 12 & 21.8 \\
\hline Bavaria & 2 & 3.6 \\
\hline \multicolumn{3}{|l|}{ Number of markets $(n=53)$} \\
\hline 1 market & 30 & 56.6 \\
\hline More than one market & 23 & 43.4 \\
\hline \multicolumn{3}{|l|}{ Number of employees $(n=54)$} \\
\hline Less than 100 employees & 40 & 74.0 \\
\hline Between 101 and 300 employees & 9 & 16.7 \\
\hline More than 300 employees & 5 & 9.3 \\
\hline \multicolumn{3}{|l|}{ Sales area $(n=51)$} \\
\hline Less than $1,000 \mathrm{~m}^{2}$ & 7 & 14.3 \\
\hline $1,001 \mathrm{~m}^{2}-2,500 \mathrm{~m}^{2}$ & 37 & 75.5 \\
\hline Over $2,501 \mathrm{~m}^{2}$ & 5 & 10.2 \\
\hline \multicolumn{3}{|l|}{ Age $(n=45)$} \\
\hline $18-25$ years & 3 & 6.7 \\
\hline $26-40$ years & 20 & 44.4 \\
\hline $41-65$ years & 22 & 48.9 \\
\hline \multicolumn{3}{|l|}{ Gender $(n=55)$} \\
\hline Female & 17 & 30.9 \\
\hline Male & 38 & 69.1 \\
\hline
\end{tabular}


Appendix 2. Past and Future Product Range Decisions Regarding Grass-fed Beef

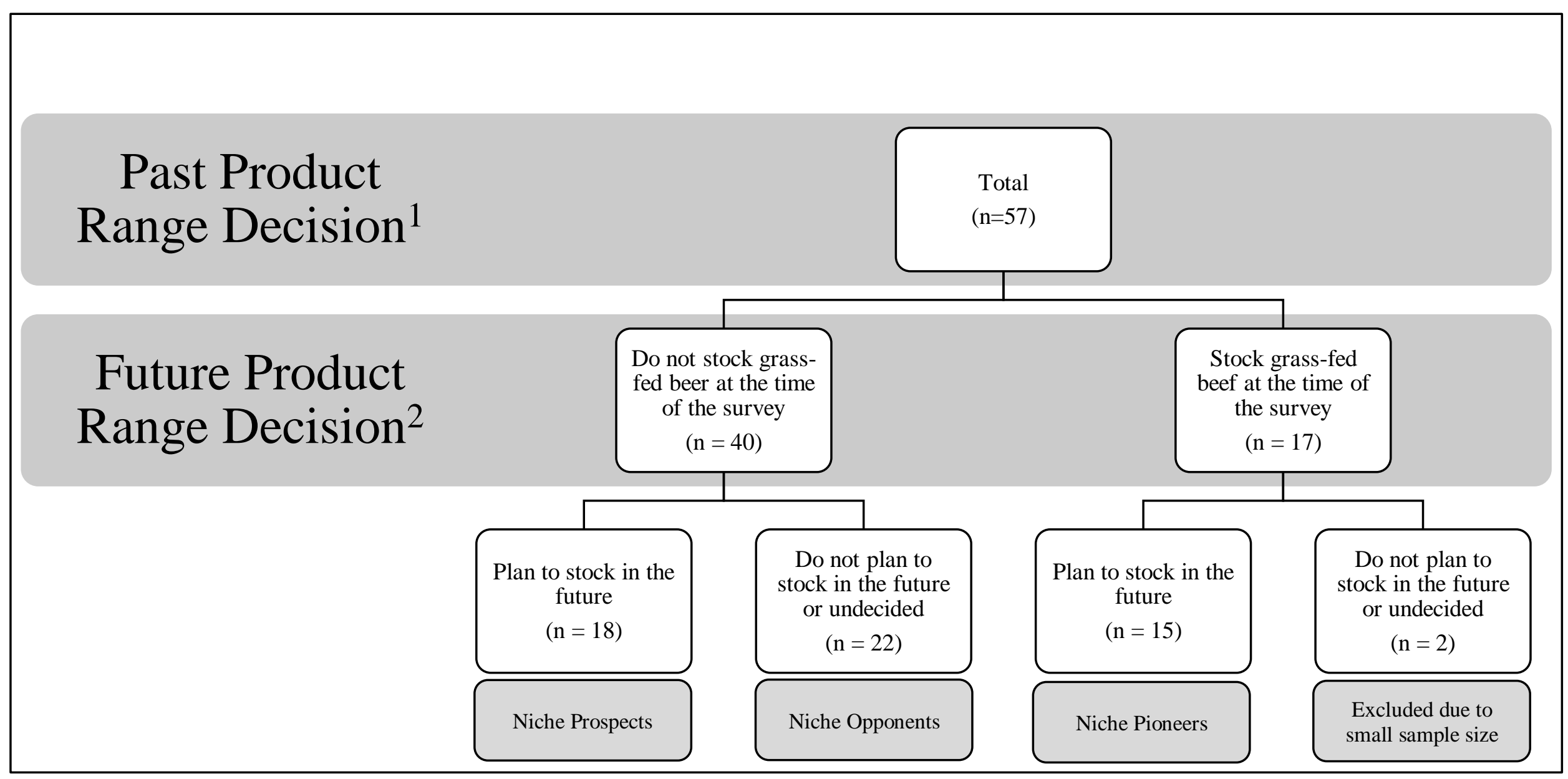

${ }^{1}$ based on: Do your product range currently include grass-fed beef?; 1 = 'no, I cannot imagine including it in the future', $2=$ 'no, but I am considering introducing it', $3=$ 'no, but I have tried it in the past', $4=$ 'yes, with moderate success', $5=$ 'yes with considerable success'. ${ }^{2}$ based on: How probable is it that you will stock (or continue to stock) grass-fed beef in 2020?; 1 = 'very unlikely', 2 = 'improbable', 3 = 'undecided', 4 = 'probable', 5 = 'very probable". 


\section{Resümee und Ausblick}

Ziel der vorliegenden Arbeit ist es, ein tieferes Verständnis für die gesellschaftlichen Anforderungen an eine Transformation der modernen Nutztierhaltung zu entwickeln sowie Gestaltungsmöglichkeiten des Lebensmitteleinzelhandels aufzuzeigen.

\section{Gesellschaftliche Wahrnehmung heimlicher Stallaufnahmen}

Der erste Teil der vorliegenden Dissertation zeigt, dass heimliche Stallaufnahmen aus gesellschaftlicher Perspektive im Schnitt als legitim wahrgenommen werden und eine härtere strafrechtliche Ahndung eher abgelehnt wird. Dies gilt sowohl für heimliche Aufnahmen, die Tierschutzvergehen aufdecken als auch für Aufnahmen, die gesetzeskonforme Haltungsbedingungen zeigen. Getrieben wird die gesellschaftliche Zustimmung vor allem durch das wachsende Interesse am Wohlergehen landwirtschaftlicher Nutztiere. Die vornehmlich als gewinnorientiert wahrgenommene landwirtschaftliche Nutzierhaltung wird hingegen als wenig vertrauenswürdig eingestuft. Negative Konsequenzen heimlicher Aufnahmen für Landwirte finden kaum Berücksichtigung in der gesellschaftlichen Meinung. In der gesellschaftspolitischen Diskussion um heimliche Aufnahmen, die die Diskrepanz zwischen Gesellschaft und Landwirtschaft darstellt, spiegelt sich auch die gesellschaftliche Dringlichkeit einer Anpassung der bisherigen Produktionsbedingungen wider. Die sich daraus ableitbaren gesellschaftlichen Anforderungen an einen Transformationsprozess der intensiven Nutztierhaltung sollen im Folgenden betrachtet werden.

Da teilweise gesetzeswidrig entstandenes Bildmaterial als legitimes Mittel zur Aufdeckung von Tierschutzverstößen wahrgenommen werden, ist von einer gesellschaftlich geforderten Überarbeitung der bestehenden Kontrollmechanismen auszugehen. Der gesellschaftliche Vertrauensverlust in die Fleischwirtschaft und Kontrollmechanismen (Schulze et al., 2008; Albersmeier \& Spiller, 2010), scheint zudem nicht allzu weit von den tatsächlichen Defiziten des föderal organisierten Kontrollsystems abzuweichen (Antwort der Bundesregierung, 2018). Eine Erhöhung der Kontrollintervalle scheint dabei ebenso, wie auch eine Verbesserung der Qualität der Kontrollen notwendig. Wie Beitrag I.2 werden dabei auch innovative Ansätze wie z.B. Whistleblowing, aus gesellschaftlicher Perspektive akzeptiert und nützlich sein, denn Whistleblowing liefert allägliche Einblicke in die Produktionspraktiken. Werden die Informationen von Whistleblowern sachgerecht und vertrauenswürdig behandelt, kann damit eine zielführende Unterstützung der bestehenden Kontrollinstanzen, die bereits zunehmend auf 
Interesse im Lebensmittelsektor zur Aufdeckung von Missständen stößt, erreicht werden (Moy, 2018; Soon \& Manning, 2017).

Heimliche Aufnahmen werden außerdem als glaubwürdige Informationsquelle wahrgenommen. Auch daran wird das zunehmende gesellschaftliche Interesse an den Produktionsprozessen von Lebensmitteln deutlich (Clark et al., 2016; Grunert, 2006). Durch die vorwiegend hinter verschlossenen Türen stattfindende landwirtschaftliche Produktion ist der gesellschaftlich geforderte Informationsfluss über die Produktions- und Haltungsbedingungen von Nutztieren allerdings erschwert. Aus gesellschaftlicher Perspektive scheinen heimliche Aufnahmen diese Informationslücke schließen zu können. Das emotional geladene Bildmaterial ist leicht zugänglich (Kroeber-Riel \& Esch, 2011). Es bleibt im Vergleich, zu der in den letzten Jahren vor allem fachlich geführten Kommunikation von Seiten der Landwirtschaft, deutlich länger in Erinnerung. Eine gesellschaftlich akzeptierte Nutztierhaltung scheint daher auch mit einer Anpassung der Kommunikationsstrategie einherzugehen. Auf eine konkrete Ausgestaltung der Kommunikationsstrategie soll die vorliegende Dissertation aber nicht eingehen, da diese schon an anderer Stelle ausführlich betrachtet ist (z.B. Spiller et al., 2016; Busch et al., 2018). Dennoch verdeutlicht der erste Teil der vorliegenden Dissertation die Relevanz des Informationsflusses zwischen Landwirtschaft und Gesellschaft. Dadurch wird unterstrichen, dass die Kommunikation momentan vor allem von Nichtregierungsorganisationen getrieben ist. An die vorherigen Arbeiten anknüpfend (Spiller et al., 2016; Busch et al., 2018) sollte daher eine proaktive Kommunikation von Seiten der Landwirtschaft im Vordergrund stehen.

Da zunehmendes gesellschaftliches Wissen über landwirtschaftliche Produktionspraktiken aber nicht unweigerlich mit einer erhöhten gesellschaftlichen Akzeptanz einhergeht (Spiller et al., 2016), reicht eine Anpassung der Kommunikationsstrategie allein nicht aus. Gesellschaftlich werden viele gesetzeskonforme Produktionspraktiken nicht mehr akzeptiert (z.B. Busch et al., 2017; Sonntag et al., 2016). Dies zeigt sich auch an heimlichen Aufnahmen, die ebenfalls als legitim wahrgenommen werden, wenn gesetzeskonforme Produktionspraktiken gezeigt werden. Eine tatsächliche Anpassung der Produktionsbedingungen scheint daher unausweichlich. In der Vergangenheit haben heimliche Aufnahmen vor allem dazu geführt, dass die Landwirtschaft aufgrund zunehmender gesellschaftlicher Kritik begonnen hat Alternativen für besonders kritisch wahrgenommene Produktionspraktiken zu entwickeln (z.B. Töten männlicher Küken). Daher scheint eine langfristige und vorausschauende Anpassung der Produktionspraktiken und Haltungssysteme, die nicht nur auf akute gesellschaftliche Kritik reagiert, erfolgsversprechender, um gesellschaftliche Akzeptanz zu erreichen. 


\section{Konsumentenpräferenzen und Zahlungsbereitschaften für nachhaltig erzeugte Fleischwaren}

Im zweiten Teil der vorliegenden Dissertation wird deutlich, dass Rindfleisch aus Weidehaltung den Anforderungen einer gesellschaftlich akzeptierten Nutztierhaltung gerecht werden kann. Wird zusätzlich ein positiver Beitrag zum Umwelt- und Naturschutz geleistet, nimmt das Interesse der Konsumenten weiter zu. Ebenfalls attraktiv für den Konsumenten ist die Nutzung von, vor allem in der extensiven Weidehaltung verbreiteten, Zweinutzungsrassen. Im Gegensatz dazu wirkt sich die Nutzung einer reinen Fleischrasse negativ auf die Kaufentscheidung aus. Daraus lassen sich die nachfolgenden Differenzierungsmöglichkeiten für den deutschen Fleischmarkt ableiten.

Bisher ist der deutsche Fleischmarkt insbesondere durch die Vermarktung standardisierter Produkte zu geringen Preisen gekennzeichnet. Es zeigt sich aber, dass die Lebensmittelwahl einer wachsenden Bevölkerungsgruppe weniger von einem möglichst geringen Preis bestimmt ist. Wichtiger wird zunehmend auch eine nachhaltige und authentische Produktionsweise, die Produkte mit hoher Qualität liefert und einen besonderen Genuss garantiert (BMEL, 2019 \& 2020). Im Zuge dieser Entwicklung kommen Premiumlebensmitteln eine wachsende Bedeutung zu (Hartmann et al., 2016). Rindfleisch aus Weidehaltung ist aufgrund des höheren Produktpreises, der besonderen Fleischqualität sowie der traditionellen Produktionsmethoden und oftmals kleinbäuerlichen Strukturen ein solches Premiumprodukt (Sidali \& Hemmerling, 2014). Um das Marktpotenzial von Premiumfleischwaren $\mathrm{zu}$ nutzen, müssen aber Marketingmaßnahmen angepasst werden, damit Konsumenten den Mehrwert des Produktes nachvollziehen können. Dabei müssen vor allem nachhaltige Produktionsprozesse, die sich durch einen hohen Grad an Authentizität auszeichnen im Vordergrund stehen (Hartman et al., 2016). Aufgrund der natürlichen Haltungsbedingungen, stellt ,Weidehaltung ' eine erfolgsversprechende Differenzierungsmöglichkeit dar. Zudem zeigt sich, dass auch die Wahl der Rasse ein bisher kaum abgeschöpftes Marktpotential aufweist.

Bisher sind in der landwirtschaftlichen Nutztierhaltung vor allem einseitig gezüchtete Rassen vorherrschend. Sie gehen aber zum Teil mit Einschränkungen des Wohlergehens der Tiere einher. Mit der Nutzung von Zweinutzungsrassen hingegen können ethisch fragwürdige Produktionspraktiken und negative Auswirkungen auf das Wohlergehen landwirtschaftlicher Nutztiere minimiert werden. Informationen über die Verwendung einer Zweinutzungsrasse scheinen daher im Zuge des zunehmenden gesellschaftlichen Interesses an tierfreundlichen und authentischen Lebensmitteln ein erfolgsversprechendes Differenzierungsmerkmal darzustellen. 
Die Vermarktung von Kobe-Rindfleisch zeigt, dass Informationen über die Rasse einen enormen Wiedererkennungswert aufweisen und im Luxuslebensmittelmarketing bereits heute erfolgreich genutzt werden. Dennoch zeigt ein Vergleich mit bisherigen Untersuchen, dass allein die Angabe der Rasse nicht ausreichend ist, um den Konsumenten den Mehrwert zu kommunizieren (Scozzafava et al., 2016). Vielmehr müssen die Werte Authentizität und Regionalität mit der Rasse verknüpft und transportiert werden sowie Informationen bereitgestellt werden, die dem Kunden, die mit einer Zweinutzungsrasse verbundenen positiven Auswirkungen auf das Wohlergehen der Tiere, erklären.

\section{Der Lebensmitteleinzelhandel als Gestalter nachhaltiger Konsum- und Produktionsmuster}

Im dritten Teil der vorliegenden Dissertation wird deutlich, dass sich der genossenschaftlich organisierte Einzelhandel in der Förderung nachhaltiger Fleischwaren engagiert. Eine Vermarktung von Fleischwaren mit höheren Tier-, Umwelt- oder Naturschutzstandards und damit einhergehend höheren Produktpreisen, ist aber durch unternehmerische Unsicherheit geprägt. Am Beispiel von Weiderindfleisch zeigt sich, dass Lebensmitteleinzelhändler durch eine dezentralere Lebensmittelversorgung mit einer weniger standardisierten Wertschöpfungskette konfrontiert sind. Sie müssen Aufgaben übernehmen, die sonst von der Industrie getragen werden (z.B. Verarbeitung weniger beliebter Teilstücke). Gleichzeitig müssen aufwendige Marketingmaßnahmen entwickelt werden, um den Kunden den Mehrwert des Produktes vermitteln zu können (z.B. intensive Mitarbeiterschulungen). Da die Einführung von Nischenprodukten üblicherweise mit einem anfänglich geringen ökonomischen Erfolg einhergeht (Geels \& Schot, 2007), scheinen finanzielle Anreize nicht ausreichend zu sein, um die Listungsentscheidungen von Lebensmitteleinzelhändlern zu erklären. Es zeigt sich, dass Lebensmitteinzelhändler, die in alternative Fleischwaren investieren, vor allem durch ihr Interesse am Wohlergehen landwirtschaftlicher Nutztiere, sowie Umwelt- und Naturschutz aber auch durch ihr Interesse an der Unterstützung der ländlichen Entwicklung getrieben werden. Daraus lassen sich Gestaltungsmöglichkeiten des Lebensmitteleinzelhandels ableiten, die im Folgenden näher betrachtet werden.

Vor allem genossenschaftlich organisierte Einzelhändler, die in der Lage sind teilweise eigene Listungsentscheidungen $\mathrm{zu}$ treffen, können sowohl Konsum- als auch zukünftige Produktionsentscheidungen nachhaltig beeinflussen. Zusätzlich sorgt vor allem die direkte Zusammenarbeit mit regionalen Landwirten $\mathrm{zu}$ einer Dezentralisierung der Lebensmittelversorgung, die auch kleineren landwirtschaftlichen Betrieben, die normalerweise 
die standardisierten Anforderungen des LEHs nicht erfüllen können, die Möglichkeit eröffnet ihre Produkte durch den LEH zu entsprechend höheren Preisen vermarkten. Es zeichnet sich dennoch ab, dass es dem LEH schwer fällt vom bestehenden Standardisierungsgedanken Abstand zu nehmen. Die wenig standardisierte Produktion von Weiderindfleisch sorgt sowohl für schwankende Warenverfügbarkeiten, als auch für Qualitätsschwankungen und wird vom Lebensmitteleinzelhandel oft als Barriere wahrgenommen.

In einer Gesellschaft, in der Ernährung zunehmend zur Identitätsbildung genutzt wird (Spiller et al., 2016), kann eine Abweichung von standardisierter Massenware aber auch als Differenzierungsmerkmal genutzt werden. Schwankende Warenverfügbarkeiten und Qualitäten können als Attribut eines individualisierten Produktes genutzt werden. Dies bedeutet konkret, dass Lebensmitteleinzelhändler nicht versuchen eine möglichst standardisierte Wertschöpfungskette zu fördern, sondern z.B. Qualitätsschwankungen durch jahreszeitenbedingtes Futter (Gras/Heufütterung) oder unterschiedliche Rassen, dem Kunden als Besonderheit präsentieren. Wird für den Kunden transparent und nachvollziehbar kommuniziert, dass kleinbäuerliche Produktionen und traditionelle Methoden mit Qualitätsschwankungen einhergehen können, kann das zu mehr Verständnis beim Kunden führen und möglicherweise das Gefühl eines authentischen Produktes gefördert werden.

Dies gilt auch für die saisonal schwankenden Warenverfügbarkeiten. Im Luxusmarketing, werden z.B. künstlich geschaffene Engpässe genutzt, um das Konsuminteresse zu erhöhen (Bastien \& Kapferer, 2013). Saisonale Warenverfügbarkeit ist eine natürlich gegebene Verknappung der angebotenen Menge und kann auch in der Vermarktung von Weiderindfleisch durch den LEH genutzt werden. Strategien des bestehenden Systems müssen also nicht unweigerlich auf Nischenentwicklungen angewendet werden, um eine erfolgreiche Vermarktung zu gewährleisten. Am Beispiel der Spargel- bzw. Erdbeervermarktung zeigen sich sehr erfolgreiche Konzepte.

Damit kann der Lebensmitteleinzelhandel den Transformationsprozess maßgeblich unterstützen. Erforderlich ist dafür allerdings eine Abkehr von den bisher in der Fleischvermarktung verbreiteten „Schweinebauchanzeigen“. Um Premiumlebensmittel, wie z.B. Weiderindfleisch erfolgreich vermarkten zu können, ist eine glaubwürdige Geschichte, die die Originalität des Produktionsprozesses beschreibt wichtig (Storytelling). Die direkte Zusammenarbeit mit regionalen Landwirten kann dem Lebensmitteleinzelhandel die Vermarktung erleichtern, indem Konsumenten die Nähe zum Landwirt und seinen 
Produktionsmethoden nähergebracht werden, um so Glaubwürdigkeit und Originalität des Produktes zu unterstreichen.

\section{Fazit und Ausblick}

Aus der Nachhaltigkeitsforschung ist bekannt, dass sich eine erfolgreiche Transformation durch eine Umwandlung eines bestehenden in ein alternatives System auszeichnet (Geels \& Schot, 2007). Dabei kann gesellschaftlicher Druck eine Transformation initiieren, indem nachhaltige Nischenentwicklungen die Möglichkeit erlangen das bestehende, durch gesellschaftlichen Druck ins Wanken geratene, System zu ersetzen (Geels \& Schot, 2007).

Der gesellschaftliche Wertewandel setzt die moderne Nutztierhaltung zunehmend unter Druck und wahrgenommene Defizite des bestehenden Systems werden sogar teilweise versucht durch illegale Protestformen zu minimieren. Im Rahmen eines Transformationsprozesses sollte daher zum einen eine Anpassung der Kommunikationsstrategie, als auch eine Anpassung der tatsächlichen Produktionsbedingungen Berücksichtigung finden. Beide Teilaspekte fordern von der Landwirtschaft eine aktive Positionierung, in der sie den Transformationsprozess leitet und dabei die gesellschaftlichen Anforderungen, aber auch wissenschaftlichen Erkenntnisse berücksichtigt. Da eine Erhöhung der Tierschutzstandards oftmals mit negativen Auswirkungen auf Umwelt- und Naturschutz einhergeht (z.B. Außenklimazugang führt zu höheren Emissionen), scheint eine grundsätzliche und langfristige Anpassung der modernen Nutztierhaltung nötig. Eine mögliche Lösung ist die Reduktion der Tierbestände. Damit können weniger Tieren bessere Haltungsbedingungen ermöglicht werden, ohne weitere negative Konsequenzen für Umwelt- und Naturschutz zu initiieren. Eine Reduktion der Tierbestände würde eine Anpassung der bisherigen Strategie der Kostenführerschaft des Fleischsektors und eine Begleitung durch eine Differenzierung nach nachhaltigen Produktattributen erfordern, die mit Ansätzen aus dem Premium- und Luxusmarketing unterstützt werden könnte (Busch et al., 2018).

Eine erfolgreiche Transformation ist aber durch das Zusammenspiel aller Akteure des Fleischsektors gekennzeichnet. Die vorliegende Arbeit untersucht drei wichtige Akteure, nämlich die Gesellschaft, den Konsumenten und den LEH anhand einiger beispielhafter Entwicklungen. Rückschlüsse sind daher auf die gesellschaftlichen Anforderungen und Gestaltungsmöglichkeiten des LEHs zu ziehen. Für ein tieferes Verständnis des Transformationsprozesses ist aber ebenso die Perspektive der Landwirtschaft, der Politik und der Nichtregierungsorganisationen relevant. Zusätzlich können auch Schocks den 
Transformationsprozess beeinflussen (Geels \& Schot, 2007) und systemische Anpassungen initiieren. Jüngste gesellschaftliche Entwicklungen unter der vorherrschenden Corona Epidemie haben daher möglicherweise einen Einfluss auf den Transformationsprozess und sollten zeitnah Berücksichtigung in wissenschaftlichen Untersuchungen finden. Im Rahmen dieser Dissertation wurde untersucht, ob und inwiefern Konsumenten bereit sind die mit einer Transformation verbundenen Mehrkosten zu tragen. Dabei blieb die gesellschaftliche Akzeptanz von Steuern und Subventionen aber unberücksichtigt. Weiterhin steht bei der Betrachtung in Beiträgen II und III mit Weiderindfleisch ein Premiumprodukt im Fokus. Zwar können daraus Differenzierungsstrategien und mögliche Anpassungen der landwirtschaftlichen Nutztierhaltung abgeleitet werden, dennoch existieren auch andere Ansätze am deutschen Fleischmarkt, die sich vor allem auf eine flächendeckende Anhebung der Tierschutzstandards in der Produktion konzentrieren (z.B. Initiative Tierwohl). In weiteren Untersuchungen sollte zudem intensiver auf die gesellschaftliche Einschätzung von Auswirkungen der landwirtschaftlichen Nutztierhaltung auf Umwelt- und Natur sowie deren Zielkonflikte mit dem Tierschutz eingegangen werden. 


\section{Literatur}

Albersmeier, F. \& Spiller, A. (2010). Die Reputation der Fleischwirtschaft in der Gesellschaft: Eine Kausalanalyse. Schriften der Gesellschaft für Wirtschafts- und Sozialwissenschaften e. V., 45, 181-193.

Antwort der Bundesregierung (auf eine kleine Anfrage der Abgeordneten der FDP) (2018), Vollzug von Tier- und Verbraucherschutzrecht. Drucksache 19/3195.

Bastien, V. \& Kapferer, J.-N. (2013). More on Luxury Anti-Laws of Marketing. In: Winter, K.P. \& Hennigs, N. Luxury Marketing. A Challenge for Theory and Practice. Gabler Verlag, Wiesbaden.

BMEL (Bundesministerium für Ernährung und Landwirtschaft) (2019). Deutschland, wie es isst. Der BMEL-Ernährungsreport 2019. MKL Druck GmbH \& Co.KG, Ostbevern. https://www.bmel.de/SharedDocs/Downloads/DE/Broschueren/Ernaehrungsreport201 9.pdf?_blob=publicationFile $\& \mathrm{v}=4$

BMEL (Bundesministerium für Ernährung und Landwirtschaft) (2020). Deutschland, wie es isst. Der BMWL-Ernährungsreport 2020. MKL Druck GmbH \& Co.KG, Ostbevern. https://www.bmel.de/SharedDocs/Downloads/DE/Broschueren/ernaehrungsreport2020.pdf?_blob=publicationFile \&v $=16$

Busch, G., Gauly, M. \& Spiller, A. (2018). Opinion paper: What needs to be changed for successful future livestock farming in Europe? Animal, 12(10), 1999-2001.

Busch, G., Weary, D.M., Spiller, A., Keyserlingk, M.A.G. (2017). American and German attitudes towards cow-calf seperation on dairy farms. PLoS ONE, 12(3), e0174013.

Clark, B., Stewart, G.B., Panzone, L.A., Kyriazakis \& Frewer, L.J. (2016). A Systematic Review of Public Attitudes, Perceptions and Behaviours Towards Production Diseases Associated with Farm Animal Welfare. Journal of Agricultural and Environmental Ethics, 29(3), 455-478.

Geels, F. W., \& Schot, J. (2007). Typology of sociotechnical transition pathways. Research Policy, 36(3), 399-417.

Grunert, K.G. (2006). Future trends and consumer lifestyles with regard to meat consumption. Meat Science, 74(1), 149-160.

Hartmann, L.H., Nitzko, S. \& Spiller, A. (2016). The significance of definitional dimensions of luxury food. British Food Journal, 118(8), 1976-1998.

Kroeber-Riel, W. \& Esch, F.R. (2011). Strategie und Technik der Werbung, In: Diller, H., \& Köhler, R. Edition Marketing. Kohlhammer: Stuttgart, Germany. 
Moy, G.G. (2018). The role of whistleblowers in protecting the safety and integrity of the food supply. npj Science of Food, 2(8), 1-5.

Schulze, H., Böhm, J., Kleinschmit, D., Spiller, A. \& Nowak, D. (2008). Öffentliche Wahrnehmung der Primärverantwortung für Lebensmittelsicherheit: Eine Medienanalyse der Gammelfleischskandale. Agrarwirtschaft, 57(7), 334-345.

Scozzafava, G., Corsi, A.M., Casini, L., Contini, C. \& Mueller, S.M, (2016). Using the animal to the last bit: Consumer preferences for different beef cuts. Appetite, 96(1), 70-79.

Sidali, K.L. \& Hemmerling, S. (2014). Developing an authenticity model of traditional food specialties: does the self-concept of consumers matter? British Food Journal, 116(11), 1692-1709.

Sonntag, W. Kaiser, A., Meyer-Höfer, M. v. \& Spiller A. (2016). Wie Ansprüche der Gesellschaft in Veränderungsprozesse einbinden? Konfrontation von Verbrauchern mit Zielkonflikten aus der Schweinhaltung. Berichte über Landwirtschaft, 95(1), 1-27.

Soon, J.M. \& Manning, L. (2017). Whistleblowing as a countermeasure strategy against food crime. British Food Journal, 119(12), 2630-2652.

Spiller, A., von Meyer- Höfer, M. \& Sonntag, W. (2016). Gibt es eine Zukunft für die moderne konventionelle Tierhaltung in Nordwesteuropa? Diskussionsbeitrag, No. 1608, Georg-August-Universität Göttingen, Department für Agrarökonomie und Rurale Entwicklung (DARE), Göttingen. 


\section{Veröffentlichungs- und Votragsverzeichnis}

\section{Beiträge in peer-reviewed Journals}

Schulze, M., Risius A. \& Spiller, A. (2018). Heimliche Stallaufnahmen aus gesellschaftlicher Sicht im Wechselspiel zwischen Landwirtschaft, Tierschutzorganisationen und staatlichen Kontrollmechanismen. German Journal of Agricultural Economics, Special Issue: Future Options for Animal Husbandry in Europe, 67(4), 267-280.

Schulze, M., Spiller, A. \& Risius, A. (2019). Food Retailers as Mediating Gatekeepers between Farmers and Consumers in the Supply Chain of Animal Welfare Meat Studying Retailers' Motives in Marketing Pasture-Based Beef. Food Ethics, 3(1), 4152.

Schulze, M., Spiller, A., \& Risius, A. (2021). Do consumers prefer pasture-raised dualpurpose cattle when considering meat products? A hypothetical discrete choice experiment for the case of minced beef. Meat Science, 177, 108494.

Schulze, M., Risius, A., \& Spiller, A. (2021). Public perceptions of undercover investigations in livestock farming: An end that justifies the means? Animal Welfare, 30(1), 39-47.

\section{Weitere wissenschaftliche Veröffentlichungen}

Esbjerg, L., Brønd, K.L. \& Schulze, M. (2020). Gatekeeper for dyrevelfærd: om detailkædernes centrale rolle for udviklingen af dyrevelfærden i dansk svineproduktion. In: Tove, C., Sand $\varnothing$, S. Styrkelse Af Grisenes Velfærd Gennem Markedsdrevne Initiativer (2020). Københavns Universitet, Institut for fødevare- og ressourceøkonomi, 61-72.

\section{Konferenz- und Tagungsbeiträge}

Schulze, M. \& Risius, A. (2019). Der Lebensmitteleinzelhandel als Wegbereiter nachhaltig erzeugter Fleischwaren, Beitrag zur Tagung "Transformationsprozesse in der intensiven Nutztierhaltung, was wollen, können und müssen wir ändern", 17. und 18. Oktober in Göttingen, schriftlicher Beitrag veröffentlicht im Tagungsband zur 2. Tierwohltagung,

Schulze, M. \& Risius, A. (2019). Personal values and attitudes as predictors of food retailer's motivations to engage in animal welfare initiatives? Beitrag zur "Corporate 
Responsibility Research Conference - Rethinking Value Creation for Sustainability", 11.-13.09.2019, Tampere, Finnland.

Schulze, M. \& Risius, A. (2019). Warenverfügbarkeit als Hemmnis in der Vermarktung von Biorindfleisch - die Perspektive des Lebensmitteleinzelhandels. Beitrag zur „15. Wissenschaftstagung Ökologischer Landbau - Innovatives Denken für eine nachhaltige Land- und Ernährungswirtschaft“, Witzenhausen.

Schulze, M. Risius, A. \& Spiller, A. (2019). Heimliche Aufnahmen in landwirtschaftlichen Ställen - Gesellschaftliche Bedenken in einer rechtlichen Grauzone: Was können wir daraus lernen und gibt es Alternativen zur Verbesserung des Tierwohls? Beitrag zur BMBF-Klausurwoche,"Wohlergehen von Tieren -ethische, wissenschaftliche, und biologische Perspektiven“ vom 25.-29.03.2019 in Münster.

\section{Beiträge in praxisorientierten Zeitungen}

Fachliche Unterstützung durch Prof. Achim Spiller und Maureen Schulze bei "Der LEH macht die Spielregeln". Topagrar, 03/2019, 126-129.

Schulze, M. \& Risius, A. (2019). Biorindfleisch-Vermarktung: Perspektiven für den Lebensmitteleinzelhandel. Lebendige Erde, 03/2019, 


\section{Beiträge der Autoren}

\section{I.1 Heimliche Stallaufnahmen aus gesellschaftlicher Sicht im Wechselspiel zwischen Landwirtschaft, Tierschutzorganisationen und staatlichen Kontrollmechanismen}

Idee und Konzeption: Achim Spiller, Maureen Schulze, Antje Risius

Literaturrecherche: Maureen Schulze, Achim Spiller

Durchführung der empirischen Untersuchung: Maureen Schulze

Datenanalyse: Maureen Schulze

Diskussion der Ergebnisse: Maureen Schulze, Achim Spiller, Antje Risius

Überarbeitung: Maureen Schulze, Achim Spiller, Antje Risius

\section{I.2 Public Perceptions of Undercover Investigations in Livestock Farming: An End that Justifies the Means?}

Idee und Konzeption: Achim Spiller, Maureen Schulze, Antje Risius

Literaturrecherche: Maureen Schulze, Achim Spiller

Durchführung der empirischen Untersuchung: Maureen Schulze

Datenanalyse: Maureen Schulze

Diskussion der Ergebnisse: Maureen Schulze, Achim Spiller

Überarbeitung: Maureen Schulze, Achim Spiller, Antje Risius

II.1 Do consumers prefer pasture-raised dual-purpose cattle when considering meat products? A hypothetical discrete choice experiment for the case of minced beef.

Idee und Konzeption: Antje Risius, Maureen Schulze

Literaturrecherche: Maureen Schulze

Design des Choice Experiments: Antje Risius

Datenanalyse: Maureen Schulze, Antje Risius

Diskussion der Ergebnisse: Maureen Schulze, Antje Risius

Überarbeitung: Maureen Schulze, Antje Risius, Achim Spiller 
III.1 Food Retailers as Mediating Gatekeepers between Farmers and Consumers in the Supply Chain of Animal Welfare Meat - Studying Retailers' Motives in Marketing Pasture-Based Beef

Idee und Konzeption: Maureen Schulze, Antje Risius, Achim Spiller

Literaturrecherche: Maureen Schulze

Durchführung der empirischen Untersuchung: Maureen Schulze

Datenanalyse: Maureen Schulze

Diskussion der Ergebnisse: Maureen Schulze, Antje Risius

Überarbeitung: Maureen Schulze, Antje Risius, Achim Spiller

III.2 Challenges and Motives in Food Retail Cooperatives' Approach to Supporting Alternative Niche Paths Using the Case of Locally Produced GrassFed Beef

Idee und Konzeption: Maureen Schulze, Achim Spiller, Antje Risius

Literaturrecherche: Maureen Schulze

Durchführung der empirischen Untersuchung: Maureen Schulze

Datenanalyse: Maureen Schulze

Diskussion der Ergebnisse: Maureen Schulze, Achim Spiller, Antje Risius

Überarbeitung: Maureen Schulze, Achim Spiller 


\section{Eidesstattliche Erklärung}

1. Hiermit erkläre ich, dass diese Arbeit weder in gleicher noch in ähnlicher Form bereits anderen Prüfungsbehörden vorgelegen hat.

Weiter erkläre ich, dass ich mich an keiner anderen Hochschule um einen Doktorgrad beworben habe.

Rømø, den 01.07.2020

Maureen Schulze

2. Hiermit erkläre ich eidesstattlich, dass diese Dissertation selbständig und ohne unerlaubte Hilfe angefertigt wurde.

Rømø, den 01.07.2020

Maureen Schulze 